\title{
Generic Ownership Types for Java and the Collections Framework
}

by

Ahmed Aziz Khalifa

\author{
A thesis \\ submitted to the Victoria University of Wellington \\ in fulfilment of the \\ requirements for the degree of \\ Doctor of Philosophy \\ in Computer Science.
}

Victoria University of Wellington

2014 



\begin{abstract}
Generic programming has turned out very useful in the development of reusable software. With the Java programming language, genericity is not only meant for reusability, but also for type-safety. Java generics constrain a container object (e.g., list, hash table) to store objects of a pre-specified data type. Nevertheless, safe programming with aliasing (multiple pointers in a program may point to the same object) is still a concern in objectoriented programming language research. A pointing object can mutate the state of the aliased object, reflecting the changes to all of the other pointers (aka aliases) thus affecting their behaviour. As programs grow larger and more complex, such changes in behaviour can be undesirable and difficult to detect and reason about. With respect to container objects, the iterator pattern critically violates encapsulation, allowing aliases to the state (and thereof the components) of its container.

Object ownership is one of the well-researched paradigms in the area of alias management. Ownership types support hierarchical object encapsulation rather than the traditional class-level encapsulation. This thesis introduces an extension of Java 6 with support for ownership types as supplementary generic types. That is, Java generics are extended with the ability of carrying ownership information. This extension provides generic ownership support for all of Java; that is, all major language features are addressed so that programs can safely manage and express their aliasing properties. The resulting language is expressive enough to support common programming idioms, with little programming and runtime overhead. We evaluated the programmability of the language by refactoring a major (the most essential) portion of the Java Collections Framework. We also evaluated the performance impact of our refactoring by conducting a small micro-benchmark study to measure the performance time overhead the refactored collections may impose.
\end{abstract}




\section{Acknowledgments}

I wish to express my gratitude to my supervisors Dr. Alex Potanin and Prof. James Noble. To Alex for his tremendous guidance, patience and support throughout this research. Alex has been habitually knowledgeable, alert and enthusiastic. I am exceedingly thankful to him for keeping me financially secure at all times during the period of this research. James, a million thank you is not enough for you. You enlightened the path forward when it seemed headed for a dead-end. I appreciate the lessons I learned from your red ink. No fair person can fail to see your insightful conjecture.

I wish to express my gratitude to A/Prof. Lindsay Groves for looking after my progress when James and Alex were on another continent; for understanding my stress and press during the final write-up.

A big thank you should go to my examiners for their time, effort and feedback. Another big thank you to the Faculty of Graduate Research for awarding me a Victoria PhD Submission Scholarship, and for the free regular workshops that helped me cope and settle with more ease. I would also like to thank Victoria International for the help given to me since the moment I put my foot on Wellington airport.

My wife Salma has been a fruitful source of love and support throughout my studies. My daughters Ola and Rana, thank you for being quiet (at night) while I was working.

My parents have always supported me to pursue my dreams. They have done everything possible to secure me the best way of life. What I owe them is countless and eternal. 


\section{Contents}

$\begin{array}{lll}1 & \text { Introduction } & \mathbf{1}\end{array}$

1.1 Contributions . . . . . . . . . . . . . . . 3

1.2 Outline . . . . . . . . . . . . . . . . . . 4

2 Background $\quad 5$

2.1 Object Sharing . . . . . . . . . . . . . . . . 5

2.2 Alias Control . . . . . . . . . . . . . . . . . 9

2.2 .1 The Geneva Convention . . . . . . . . . . . . . . 9

2.2.2 Alias Transitivity and Aggregation . . . . . . . . . . 10

2.2 .3 Uniqueness . . . . . . . . . . . . . . 12

2.2 .4 Full Alias Protection . . . . . . . . . . . . . . 14

2.2 .5 Confined Types . . . . . . . . . . . . . . . . . 15

$2.2 .6 \quad$ Flexible Alias Protection . . . . . . . . . . . . . . 16

2.3 Object Ownership . . . . . . . . . . . . . . . . 17

2.3 .1 Deep Ownership . . . . . . . . . . . . . . . 18

2.3 .2 Universes . . . . . . . . . . . . . . . 21

2.3 .3 External Uniqueness . . . . . . . . . . . . . . 22

2.3 .4 Ownership Generic Java (OGJ) . . . . . . . . . . . 23

2.3 .5 Wildcards and Generic Ownership . . . . . . . . . . . . 25

2.3 .6 Generic Universe Types (GUT) . . . . . . . . . . . . 25

$\begin{array}{lll}3 & \mathbf{O G J}_{+} \text {Language } & 27\end{array}$

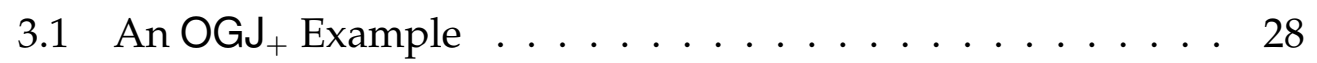


3.2 Reference Types . . . . . . . . . . . . . . . . . . . 30

3.3 Class Hierarchy . . . . . . . . . . . . . . . . . . . . . 32

3.3 .1 Subtyping . . . . . . . . . . . . . 33

3.3 .2 Interfaces . . . . . . . . . . . . . . . . . . . 34

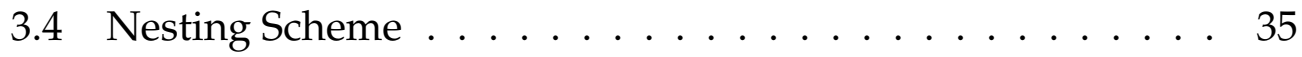

3.4 .1 Class Declaration . . . . . . . . . . . . . . . . . . 35

3.4 .2 Field/Variable Declaration . . . . . . . . . . . . . 35

3.4 .3 Method Declaration . . . . . . . . . . . . . . 36

3.5 Instantiation and Casting . . . . . . . . . . . . . . . . . 38

$3.6 \quad$ Wildcard Types $\ldots \ldots \ldots \ldots$. . . . . . . . . . . . . . 40

$3.6 .1 \quad$ Ownership Context Covariance. . . . . . . . . . . . . 41

3.6 .2 Readonly References . . . . . . . . . . . . . . . 44

4 From Java To OGJ

4.1 Arrays . . . . . . . . . . . . . . . . 47

4.1 .1 The main () Method . . . . . . . . . . . . . . 48

4.2 Inner Classes . . . . . . . . . . . . . . . . . . . . . 50

4.3 Statics . . . . . . . . . . . . . . . . . . . . . 55

4.3 .1 Static Fields . . . . . . . . . . . . . . . . . 56

4.3 .2 Static Methods . . . . . . . . . . . . . . . . . . . 57

4.3 .3 Static Blocks and Nested Static Classes . . . . . . 57

4.4 Clone . . . . . . . . . . . . . . . . . . . . . . 58

4.5 Equals . . . . . . . . . . . . . . . . 64

4.6 Exception Handling. . . . . . . . . . . . . . . . . . . . . . 68

4.7 Enum Types $\ldots \ldots \ldots \ldots$. . . . . . . . . . . . . . 71

4.8 Implementation Methodology . . . . . . . . . . . . . . 73

$4.8 .1 \quad$ Ownership Domains and Types … . . . . . . 73

4.8 .2 Type Checking . . . . . . . . . . . . . . . 74

4.8 .3 Testing . . . . . . . . . . . . . . . . . . . . 76

$4.8 .4 \quad$ Usage . . . . . . . . . . . . . . . . . . . . . . 77 
CONTENTS vii

\begin{tabular}{|lll}
5 & Generic Ownership Compliant Collections & 79
\end{tabular}

5.1 Interfaces . . . . . . . . . . . . . . . . . 83

5.1 .1 The collection Interface . . . . . . . . . . . . 84

5.1 .2 The Map Interface . . . . . . . . . . . . . . . . 90

5.1 .3 The Iterator Interface . . . . . . . . . . . . 92

5.2 Lists . . . . . . . . . . . . . . . . . . . . . . . . 94

$5.2 .1 \quad$ Array List $\ldots \ldots \ldots$. . . . . . . . . . . 95

5.2 .2 Linked List . . . . . . . . . . . . . . . . . 997

$5.2 .3 \quad$ Vector $($ legacy $) \ldots \ldots \ldots$. . . . . . . . . 100

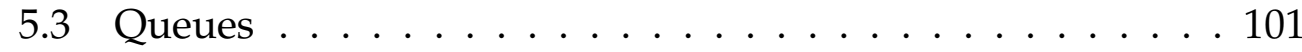

$5.3 .1 \quad$ Priority Queue . . . . . . . . . . . . . . . . . . 101

$5.3 .2 \quad$ Array Deque . . . . . . . . . . . . . . . 102

$5.4 \mathrm{Maps} \ldots \ldots \ldots \ldots . \ldots \ldots 3$

$5.4 .1 \quad$ Hash Map . . . . . . . . . . . . . . . . . 104

5.4 .2 Linked Hash Map . . . . . . . . . . . . . . . . 106

5.4 .3 Hash Table (legacy) . . . . . . . . . . . . . . . . . . . . 109

5.4 .4 Identity Hash Map . . . . . . . . . . . . . . . . . . . . 111

5.4 .5 Tree Map . . . . . . . . . . . . . . . . . . 112

5.5 Sets . . . . . . . . . . . . . . . . . . . 113

5.5 .1 Hash Set . . . . . . . . . . . . . . . . . 114

5.5 .2 Linked Hash Set . . . . . . . . . . . . . . . . . 114

5.5 .3 Tree Set . . . . . . . . . . . . . . . . 115

5.6 Usability . . . . . . . . . . . . . . . . . . . 116

$\begin{array}{lll}6 & \text { Evaluation } & 119\end{array}$

6.1 IteratorLoops . . . . . . . . . . . . . . . . 120

6.2 Collectiontoops .................... 122

6.3 MapLoops ........................... 126

6.4 MapMicroBenchmark . . . . . . . . . . . . . . . . 127

6.5 Discussion . . . . . . . . . . . . . . . . . . . 128 
\begin{tabular}{lll}
\hline 7 & Conclusions & 131
\end{tabular}

7.1 Limitations . . . . . . . . . . . . . . . . . . . . . 132

7.2 Future Directions $\ldots \ldots \ldots$. . . . . . . . . . . 133 


\section{List of Figures}

2.1 Shared List. . . . . . . . . . . . . . . . . . . . . . . . . 6

2.2 Representation Exposure Example. . . . . . . . . . . . . . 7

2.3 Types of references. Object aggregate is denoted by the box. 11

2.4 Ownership Tree with Sharing Contexts . . . . . . . . . . 17

2.5 Deep Ownership Relationships Between Objects. . . . . . . . 19

2.6 References must not cross a context boundary from the outside to the inside . . . . . . . . . . . . . . . 21

2.7 Universe Types Modifier Combinator. . . . . . . . . . . . 22

2.8 Ownership Transfer of an Externally Unique Object . . . . . 23

$3.1 \quad$ A generic ownership compliant version of Fig. 2.2. . . . . . . 29

3.2 A possible $O \mathrm{OJ}_{+}$class header. . . . . . . . . . . . . 30

3.3 Using a naked owner to infer ownership information. . . . . 31

3.4 OwnedArray class header. . . . . . . . . . . . . . . . 32

3.5 Context information must be preserved. . . . . . . . . . . . 33

$3.6 \quad$ Illegal Owner Nesting. . . . . . . . . . . . . . . . . . . . . 36

3.7 Context boundaries should not be broken through method invocations. . . . . . . . . . . . . . . 37

$3.8 \quad$ Preserving Ownership Information $\ldots \ldots \ldots \ldots \ldots$

3.9 Preserving Ownership Information against Up/DownCasting 39

3.10 Generic Ownership Wildcard Types . . . . . . . . . . . . 43

3.11 Restrictions on method invocations via wildcard owner parameterised receivers $\ldots \ldots \ldots \ldots$ 
3.12 Wildcard owned references are readonly . . . . . . . . . . . . 45

4.1 The Use of OwnedArray . . . . . . . . . . . . . . . . . . . . . 49

4.2 No common representation between inner classes and outer

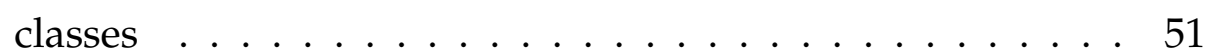

4.3 An outer class's owner is not inside the inner class's owner. 52

4.4 Event-Listener Pattern implemented in OGJ (uncompilable) 53

4.5 Event-Listener Pattern implemented in OGJ (compilable) . 54

4.6 Illegal Static Field Declaration . . . . . . . . . . . . . 57

4.7 The ArrayList's override clone () method. . . . . . . . . 60

4.8 An unreal OGJ ${ }_{+}$version of ArrayList's clone () . . . . . 61

4.9 The OGJ + ArrayList's deep cloning operation . . . . . . . 62

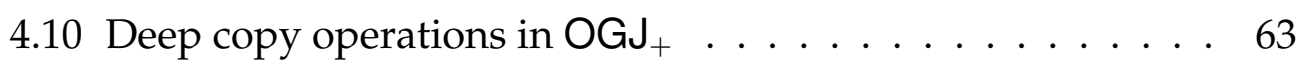

4.11 java.util.AbstractList.equals (). . . . . . . . . 64

4.12 OGJ.java.util.AbstractList.equals() . . . . . 65

4.13 equals () accessing two unrelated private objects . . . . . . 66

4.14 Illegal equals ( ) invocation (receiver and actual parameter are wildcard-owned) . . . . . . . . . . . 667

4.15 Exception Handling Example . . . . . . . . . . . . . . . 69

4.16 Depiction of the call stack exemplified in Fig. 4.15 . . . . . . 69

4.17 Depiction of the object ownership structure exemplified in Fig. 4.15 . . . . . . . . . . . . . . . 69

4.18 Mandatory declarations required for a minimal runnable $\mathrm{OGJ}_{+}$program ................. 77

5.1 Iterable Collections . . . . . . . . . . . . . . . . 81

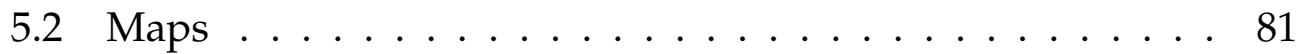

5.3 JDK 1.6 Collection Interface. . . . . . . . . . . . . . 84

$5.4 \mathrm{OGJ}_{+}$Collection Interface. . . . . . . . . . . . . . . 85

5.5 A liberal signature of contains () . . . . . . . . . 87

$5.6 \mathrm{OGJ}_{+}$AbstractCollection.removeAll (). . . . . . . 88

5.7 Arraylist constructor takes a Collection argument . . 89 


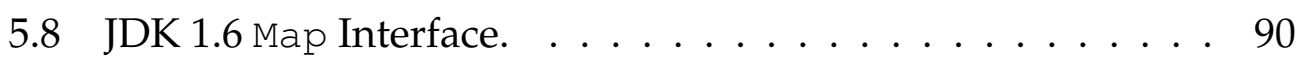

$5.9 \mathrm{OGJ}_{+}$Map Interface. . . . . . . . . . . . . . . . . . . . 91

5.10 Linked List's Iterator as an Inner Class . . . . . . . . . . . . 93

$5.11 \mathrm{OGJ}_{+}$List Interface. . . . . . . . . . . . . . . . . . 94

5.12 Linked List with Iterator . . . . . . . . . . . . . . . . . 97

5.13 LinkedList Fields and Constructors . . . . . . . . . . . . . 98

5.14 An object of an anonymous class as a sibling of the enclosing object . . . . . . . . . . . . . . . 100

5.15 Enumeration implemented as an anonymous class in the same context as Vector . . . . . . . . . . . . . . . 101

5.16 Hash Map with Iterator . . . . . . . . . . . . . . . 105

5.17 Java 1.6 LinkedHashMap.Entry.recordAccess () . . 108

5.18 A HashMap storing multiple values for the same key using Arraylist. . . . . . . . . . . . 116

$5.19 \mathrm{OGJ}_{+}{ }^{\prime}$ s version of Fig. 5.18 . . . . . . . . . . . . . 117

6.1 IteratorLoops - Nanoseconds per Iteration Step . . . . . 121

6.2 CollectionLoops for Lists and Queues - Nanoseconds per Operation . . . . . . . . . . . . . . . 123

6.3 CollectionLoops for Sets - Nanoseconds per Operation . 124

6.4 MapLoops - Nanoseconds per Operation . . . . . . . . . . 126

6.5 MapMicroBenchmark - Nanoseconds per Operation over a map size of $589,824 \ldots$. . . . . . . . . . . . . 127

6.6 MapMicroBenchmark's results for JDK 1.6 HashMap . . . . 128 


\section{Chapter 1}

\section{Introduction}

Since the 1940s to the end of the 1980s, software construction had evolved considerably. Since the 1990s to the present, software construction has evolved miraculously. One of the most prominent advances in the last three decades is the evolution of object-oriented technologies. Objectoriented techniques are mainly meant for reusability, or building reusable components, thus less software needs to be written. Nevertheless, building reliable software components is still a challenge.

The value of some constituent object-oriented techniques such as inheritance, polymorphism and dynamic binding was not well-known when the information hiding principle [54, 55] and the encapsulation technique [40, 41] were introduced. Heretofore, encapsulation is the most distinguishing feature of object-orientation [26]. A data abstraction is an object whose state is accessible only through its operations [66]. Encapsulation evolved with the introduction of inheritance to include, along with the notion of data abstraction, the ability to hide references to subobjects from the clients of the inherited object. This evolution is in fact related to the area of alias management.

Reference aliasing is the property that allows multiple objects to refer to a single object which can be changed by any of the referrers. Aliasing is inevitable in object-orientation. Object-oriented languages without aliasing 
are inefficient, if not powerless. Yet, aliasing is a major source of complications, and an important source of malfunctioning in object-oriented systems. The misuse of aliasing breaks the encapsulation needed for building reliable software components [31, 39]. Therefore, aliasing must be managed.

Object ownership [21] is one approach to alias management. This approach restricts the interplay between aliasing and the mutable state of an object, so that an incoming reference cannot penetrate from the outside of that object to directly access its internal state. This is done by identifying an owner for every individual object in a system, and by allowing modifications only through the object's owner. Deep ownership [17, 19] is one of the kinds of object ownership, which lays out the heap into nested constructs. Generic ownership [61] is the merging of deep ownership types with parametric polymorphism.

Potanin et al. [61] provide a formal model for combining deep ownership information with Java generics. Potanin et al. also provide an extended version of Java, named Ownership Generic Java (OGJ). This prototype addresses the basic features of Java, such as class declaration, method parameterisation and field access, to show how ownership information can be merged into generic declarations and how restrictions can be imposed statically.

In this thesis, we concentrate on Generic Ownership in the context of Java. The popularity of Java and the vivid alias management research induce the research problem of this thesis: What are the potentials and limits of combining deep ownership with Java generics in a real language implementation that addresses all features of Java? The implementation of Java generics does not touch the Java Virtual Machine (JVM) or the class file structure, what are the possibilities of keeping them untouched without compromising the generic ownership encapsulation? Deep ownership is too strict to support constructs like iterators, how could we support iterators without compromising encapsulation? Finally, what are the poten- 
tials and limits of our language's usability in terms of programmability and performance?

\subsection{Contributions}

This thesis makes the following contribution:

- $\mathbf{O G \mathbf { J } _ { + }}$ is an extension of Java 6 with support for Generic Ownership Types. $\mathrm{OGJ}_{+}$provides ownership support for all features of Java. To provide support for constructs such as iterators, $\mathrm{OGJ}_{+}$introduces readonly references using the wildcard feature of Java generics. $O G J_{+}$utilises wildcards to relax the restrictions imposed by the deep ownership model without compromising the nested structure of the heap.

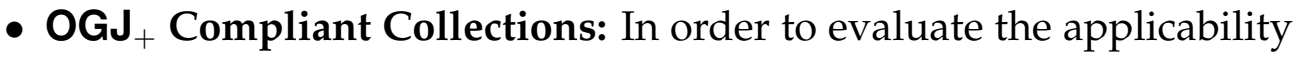
of $\mathrm{OGJ}_{+}$'s encapsulation system to a real life code base, we refactored the complete set of general purpose implementations of the JDK 1.6 Collections Framework. In addition to the ten general purpose implementations, we refactored two legacy implementations and one special purpose implementation. All of these implementations are decedents of five abstract data types: List, Queue, Deque, Map, and Set. These interfaces were refactored along with the abstract classes that provide their skeleton implementations. In total, we have refactored 38 classes and interfaces.

- A Micro-Benchmark Study: In order to evaluate the refactored collections in terms of performance, we conducted a micro-benchmark study quantifying the overall impact of emplacing ownership information into the declarations of Java generics. 


\subsection{Outline}

The next chapter provides a background on the problem of aliasing and representation exposure in object-oriented programming. Thereafter, we review some of the proposals that address this problem, showing different forms of alias management. Finally, we focus on object ownership, providing background on the attempts which inspired our work.

Chapters 3 and 4 introduce the $O G J_{+}$extension of Java. Both chapters give a detailed explanation of the various features of $O G J_{+}$, with the necessary discussion on how each feature deals with aliasing.

Chapter 5 describes the implementation details of applying generic ownership to the Collections Framework, with the necessary discussion on the programmability of $\mathrm{OGJ}_{+}$.

Chapter 6 quantifies the cost of our encapsulation system, measuring the runtime overhead imposed by the $O G J_{+}$collections on a set of microbenchmarks implemented specially to target the Java Collections Framework. We discuss the pros and cons of the integration of object ownership into Java generics.

Finally, Chapter 7 concludes with a summary and directions for future work. 


\section{Chapter 2}

\section{Background}

\subsection{Object Sharing}

Sharing objects is fundamental to object-oriented programming [39, 42]. According to Wegner [66], 'a language is object-based if it supports objects as a language feature'; 'an object-based language is object-oriented if its objects belong to classes and class hierarchies may be incrementally defined by an inheritance mechanism'; 'a class is a template (cookie cutter) from which objects may be created (instantiated) by create or new operations'; 'objects of the same class have common operations and therefore uniform behaviour'. Declaring a class creates a unique type that can be called class type, user-defined type or reference type. In a mainstream object-oriented language such as Java, variables of class types are references to objects. That is, a variable of a class type holds the memory address of where the actual class instance (object) is stored; and therefore, assignments to variables of class types copy the references only. These assignments are said to have reference semantics. Reference semantics make object sharing attainable and make efficient use of the heap. Consider Fig. 2.1. variables $x$ and $y$ are of the same class type that represents a list of integers. Variable $x$ refers to a list whose members are the first four positive integers. The assignment statement $\mathrm{y}=\mathrm{x}$ does not make a copy of $\mathrm{x}$, 
but makes y refer to the same list referred to by $\mathrm{x}$. The change in $\mathrm{y}$ also changed $x$. References $x$ and $y$ are said to be aliases, of one another, for the same object. An object is aliased whenever it is referred to by more than one other object.

$$
\begin{aligned}
& x=[1,2,3,4] \\
& y=x \\
& y \cdot \operatorname{add}(5)
\end{aligned}
$$

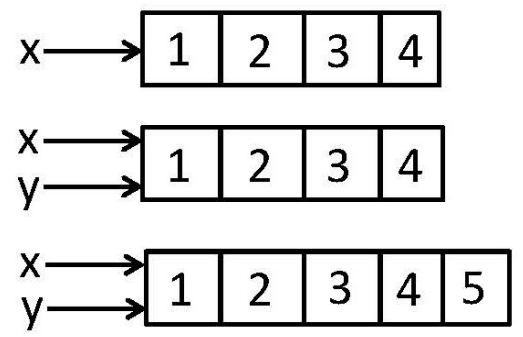

Figure 2.1: Shared List.

Reference semantics are the only possible approach to sharing objects in mainstream object-oriented languages. While sharing is an empowering feature in terms of heap utilization and in the design of data structures, misusing reference aliasing can lead to unexpected program behaviours. Such behaviours can be very difficult to understand and reason about as the system grows.

Consider the example in Fig. 2.2, in line 11 the field wPos forms the private state of class Widget; wPos is of type Point; and Point is what we call a reference type or class type. That is, the data referred to by wPos is always an instance of class Point, and represents the position of an individual widget instance (say $w 1$ ). Sharing this data with any other Widget instance (say w2) will result in dragging $w 1$ behind $w 2$ wherever $w 2$ moves, and vice versa. That is why wPos is declared private, meaning that the position data of any widget instance is not sharable. Consider the instantiations in lines 22 and 24, if we try to have an assignment statement such as $\mathrm{w} 1$. wPos=pos, we will get a compile time error saying wPos has private access in widget. This is not enough, however. Consider the benignlooking method invocations in line 26, method getPosition() returns wPos; which means that w1.getPosition() is the same as w1.wPos, 


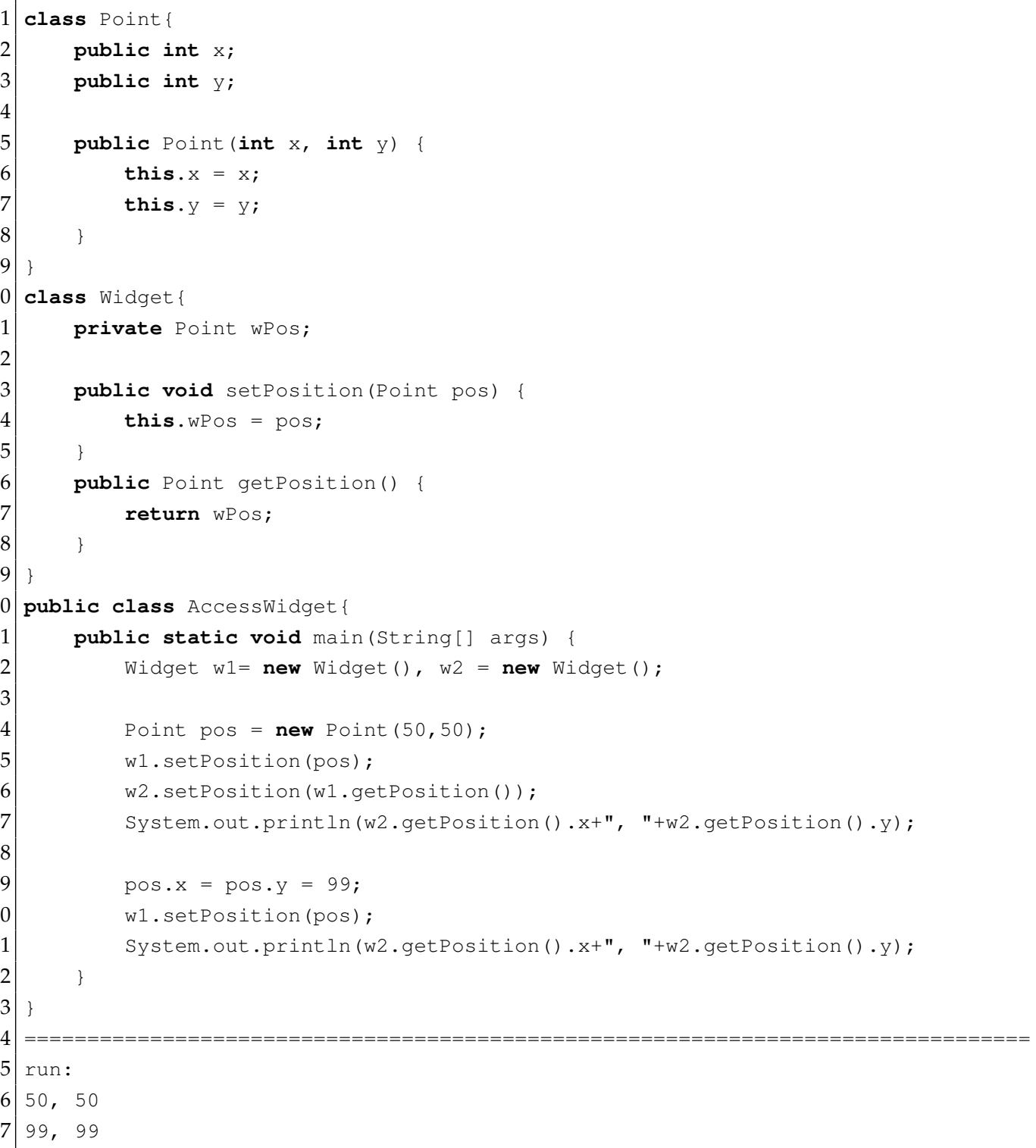

Figure 2.2: Representation Exposure Example.

but Java allows this method invocation. That is, wPos is not properly hidden or encapsulated inside Widget; and as a result, object w2 managed to share the position data of $w 1$. That is, the method invocations in line 26 are in fact a workaround the disallowed statement $\mathrm{w} 2$. wPos=w1. wPos, by 
means of public getter/setter methods. Java's class-level private fields restrict access to the names of the local state, but does not restrict access to the objects to which the names refer. An object's private state or internal representation [52] can still be accessed and modified by its public methods that can directly return references to that private representation.

The principle of information hiding [54, 55] was introduced as a design criterion in modular programming. Information hiding means that independent information (wPos) should be hidden in independent modules (Widget). That is, as long as programmers have as little code dependencies as possible, development risks are diminished. In object-orientation, independent information or the internal representation (aka the private state) should be accessed only through operations (getPosition() \& setPosition ()) which are grouped together with the internal representation in a data abstraction (Widget). This grouping property in objectoriented languages is what we call Encapsulation [6, 26, 39, 40, 41, 42]. Our example followed these principles; the operations of the data abstraction were the only access mechanism to the internal representation. Nevertheless, the encapsulation was easily breached.

Much of the flexibility and efficiency of object-oriented coding is grounded by the notion of sharing mutable objects. Nevertheless, the unrestricted interplay between aliasing and the mutable state can lead to unfavourable outcomes. What happened in the example above is that a supposedly hidden object, wPos, was exposed. This phenomenon of representation exposure [20, 22, 39, 46] is the side-effect of reference semantics. Representation exposure is the inability of an object to prevent references to objects that make up its internal representation from leaking outside its boundary. That is, representation exposure occurs as long as an object lacks the capacity to constrain aliasing sufficiently in order to enforce encapsulation.

One of the most expressive comments on the situation described in this section came from John Hogg [30]: 
"Object-oriented languages have a light side and a dark side. The light side is that the programming model makes rapid prototype implementation much easier, since components can be easily reused. The dark side is that as these prototypes mature, the components can manifest strange behaviors due to unforeseen interactions and interrelationships. The big lie of object-oriented programming is that objects provide encapsulation".

\subsection{Alias Control}

Protecting values from being changed due to aliasing has been a research concern since the very early 1990s. In this section, a brief review of literature on the alias management research is provided. Since this thesis work is set in the context of ownership types, we allocate the next section for a general overview of object ownership.

\subsubsection{The Geneva Convention}

The Geneva Convention on the Treatment of Object Aliasing [31] describes aliasing as a problem for both formal verification and practical programming. It sorts out four approaches to dealing with aliasing: detection, advertisement, prevention and control.

Alias detection is subsequent to program implementation. Alias detection is the process of detecting actual and potential aliasing using static and dynamic techniques. The resulting information will classify each object as never, sometimes, or always aliased by any two variables.

Alias advertisement keywords can be used to annotate methods on the basis of their resulting aliasing properties, so that modular analysis can be made more efficiently. Alias advertisement is desirable for the reason that comprehensive detection is impractical. 
Alias prevention is the act of verifying statically that aliasing is prohibited within confirmed contexts. This requires conservatively defined static constructs. Eliminating aliasing within particular contexts can help reason about the validity of programs.

Alias control is the act of adopting techniques capable of preventing a system from reaching the level of causing unexpected aliasing. Alias control is the most appreciated approach to dealing with aliasing, since prevention hinders the flexibility of object structures. According to Hogg et al. [31], there are situations in which the dynamic state of the system needs to be taken into consideration in oreder to pin down the bad effects of aliasing. Therefore, alias control needs to be applied at the programming level.

\subsubsection{Alias Transitivity and Aggregation}

An aggregate is an abstraction for an object that represents a storage destination for a collection of other objects (e.g., a list, table or bag). Noble et al. [52] describe the problem of representation exposure in the context of aggregation. An aggregate object's state is likely to change via an alias to any of the component objects that make up the aggregate's representation, while the aggregate itself is unaware of any aliasing. An aggregate is considered unaware of the aliases of its representation whenever an external object manages to bypass the aggregate's interface operations and directly refers to a state object. Any reference from the outside to the inside of an aggregate can mutate the state of the object's implementation, either by modifying a field or via method calls.

Clarke [16] classifies references, in the context of aggregation, into four categories: internal, external, outgoing and incoming references; or into benign references (internal and external) and problematic references (outgoing and incoming). 
Internal references are those created inside the aggregate object, referring to internal objects, and solely to interact internal to the aggregate's implementation. They are safe as long as they are not depending on outgoing references or responsive to incoming aliasing. See references $a \rightarrow b$ and aggregate $\rightarrow a$ in Fig. 2.3 .

External references are those to the aggregate itself, but neither expose nor access the internal implementation of the aggregate. See reference $x \rightarrow$ aggregate in Fig. 2.3.

Outgoing references pass from an object inside the aggregate to alias an object external to it. The aggregate has no control over external objects, while the references depend on them and there is no guarantee that the dependency is on the immutable parts of the external object [52]. See reference $\mathrm{a} \rightarrow \mathrm{y}$ in Fig. 2.3 .

Incoming references penetrate from the outside to the inside, and are considered very critical since they can directly access the internal state of the aggregate, and thus mutate it, without the aggregate being aware of the aliasing [31]. See reference $z \rightarrow b$ in Fig. 2.3 .

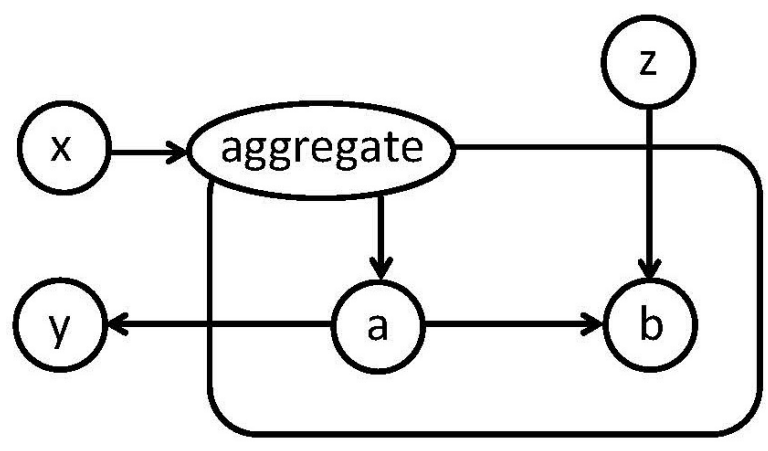

Figure 2.3: Types of references. Object aggregate is denoted by the box.

Various attempts have been proposed to prevent the problem of representation exposure, and thus eliminating the leak of references to the internal representation. In the rest of this chapter, we will highlight some of the key attempts. 


\subsubsection{Uniqueness}

An object is unique as long as it is being referred to by only one reference. If a language can constrain an object to have only one external reference, then such an object can never be aliased. Several proposals deal with different notions of uniqueness [4, 10, 11, 27, 30, 43, 65].

Class-level annotations [43] are the very basic form of uniqueness, proposed for Eiffel. In this encapsulation system, any instance of the annotated class can be referred to by only one reference. This proposal does not make clear whether uniqueness or non-uniqueness should be preserved over subtyping or not. Wrigstad [67] argues that even if uniqueness is not preserved over subtyping, uniqueness might not become invalidated. Nevertheless, non-uniqueness must be preserved over subtyping, since a non-unique superclass might involve methods that create aliases to this; invoking such methods in a unique subclass should invalidate its uniqueness. Eiffel has expanded classes [42]. An expanded type object cannot be referenced by other objects, it can only be copied. The expansion nature of a class is not transmitted via inheritance unless the subclass is declared expanded. Also, the other way around holds; a subclass inherited from a reference (non-expanded) class can be declared expanded. That is, the argument about the preservation of non-uniqueness also applies to the preservation of non-expansion.

Reference-level access control proposals [10, 11, 27, 65] demonstrate more refined and restrictive forms of uniqueness. A variable or field annotated as unique is the only possible reference in the system to a particular object. Limiting the number of references to only one reference to a particular object is simple and powerful, but not practical since unique references cannot, for example, refer to the internally aliased objects of a node-linked model, such as a doubly-linked list or a tree map. In most cases, the components of aggregate objects are internally aliased. 
Instance-level uniqueness proposals consider an object as free [30, 52] or virgin [37] if it can be initialised without being given a reference. An object's internal representation can then be set to such a free object, guaranteeing that it will not be captured in a variable. Hogg [30] provides the free aliasing mode among other aliasing modes required to characterise an object as part of an island or as a bridge capable of connecting two islands; this is discussed in subsection 2.2.4. Noble et al. [52] provide the free aliasing mode among other aliasing modes to maintain the flexibility required for alias-protected aggregates; this is discussed in subsection 2.2.6

To transfer an instance's unique reference from one place to another, additional language features are required. Almeida [4] uses copy assignments. Hogg [30] proposes an atomic operation, called destructive read, to nullify unique variables after returning their values. Boyland [10] statically checks the sharing properties of the variables to nullify them after the transfer if they hold unique references. To avoid destructive reads, Boyland[10] accommodates a borrowed qualifier so that a unique reference can be passed to a method only for the span of its operation. A borrowed reference transiently loses its uniqueness, but cannot be returned by the method or captured in fields. The unique reference is still visible during the borrowing, however.

Other language features can be associated with uniqueness. Among other capabilities, including unique capability, Boyland et al. [11] provide a null capability so that a reference with this capability can only perform instance identity comparisons; such a reference can neither access state variables nor invoke operations. Wrigstad [67] argues that references with null capability to unique objects weaken uniqueness. 


\subsubsection{Full Alias Protection}

Islands [30] and Balloons [4] control possible aliasing amongst the state objects statically. External objects are not permitted to have references to the internal representation. This subsection provides a brief overview of the aim of both proposals. This aim is considered common and termed 'full alias encapsulation' in Noble et al. [52].

The Islands proposal aims to group objects into sets of dependent objects; each set is called an island. The objects that make up an island can access each other without restrictions. Objects in one island cannot access objects in another island. Sharing between two islands can only be done through a bridge object. All parameters and results of a bridge object's operations must be read, unique or free. An interface can be annotated as: read if it is required to be only readable and not assignable to a field; unique if the object is required to have only one reference to it; or free if the object is not required to have any references.

In the Balloons proposal, data types are classified into balloon types and non-balloon types. Non-balloon types are for full freedom of sharing. Balloons cannot be accessed via state variables of any external object. Any object pointed to by a balloon is part of the balloon and hence has the same aliasing restrictions as the balloon. All objects inside a balloon have access to each other. Any instance of a balloon can be referred to by one and only one external reference; incoming references are not allowed. Copy assignments are then relied on to pass references from one balloon to another, which is expensive.

The Islands proposal relies mainly on annotations. The Balloons proposal uses a single class annotation to binary classify data types into balloon and non-balloon types; program analysis is then relied on to verify statically the sharing properties of the objects. In both proposals, fully encapsulated aggregate objects cannot share their contents between each other. To minimize restrictions, both proposals make a distinction between static aliasing (local state variables involved) and dynamic aliasing (stack 
based variables involved). Islands allow dynamic aliases to the internal representation but they are restricted to be read only; this form of dynamic aliasing does not break encapsulation. Balloons can be Opaque or Transparent. Opaque balloons do not allow any form of dynamic aliasing. Transparent balloons do not enforce any restrictions on dynamic aliasing; that is, dynamic aliases are allowed to the internal representation; this form of dynamic aliasing breaks encapsulation and exposes the internal representation.

\subsubsection{Confined Types}

The Confined Types proposal [64] is another static technique to enforce the protection boundaries required to prevent undesirable aliasing. Confinement is a package-level encapsulation scheme proposed for Java. In this proposal, any instance of a class annotated with the keyword confined cannot be referenced outside the package in which this annotated class is declared. The same applies to instances of any subclasses of the confined class.

This proposal also introduces the notion of anonymous methods to relax confines to the extent that allows flexible code reuse. Any method annotated with the keyword anon will propagate the identity of the current instance only and solely to anonymous methods; no aliases to the current instance can originate from within such a method. The flexibility provided by introducing anonymous methods is that confined classes are allowed to inherit methods from unconfined superclasses. A confined class should only call anonymous methods and non-native methods defined in other confined classes. Anonymous methods are disallowed from fetching other objects unless through variable this; but cannot assign this to a variable or method argument; and cannot return this. 


\subsubsection{Flexible Alias Protection}

Flexible alias protection [52] is another conceptual model for enforcing alias encapsulation and managing the effects of aliasing. Rather than recognizing aliasing as a problem in itself, the visibility of an object's state changes via aliases is the premise on which this model is founded. This model distinguishes between two sets of objects: private unexposed mutable representation objects, and public shareable immutable argument objects. That is, an aggregate's representation objects can be read and write, but should not be visible from the outside of the aggregate; and the aggregate's argument objects can be aliased without restrictions, but the aggregate should not depend on their mutable state. This model introduces aliasing mode declarations to annotate static types, and aliasing mode checking to statically verify the aliasing properties of an object's implementation. Aliasing modes are incorporated into a language's type expressions, resulting in moded type expressions. The proposed modes are:

rep This mode classifies an object as a private mutable representation object that is restricted from being sent out of the object to which it belongs.

$\arg \boldsymbol{R}$ This mode classifies an object as a public immutable argument object. $\mathrm{R}$ is an optional role tag used to individualize respective roles.

free This mode classifies an object as an unaliased object.

val This mode classifies an object as an instance of a value type. It has the same semantics as arg, but does not require a role.

var $\boldsymbol{R}$ This mode classifies an object as an aliased mutable object. It has the same semantics as regular references in typical object oriented languages. 


\subsection{Object Ownership}

In the light of the Flexible Alias Protection model [52], the pioneer model for ownership types was introduced by Clarke et al. [21]. Any object should be owned by only one owner object, and can only be accessed through that owner object. The set of objects owned by the same owner is named a context, since these objects have one sharing context [62]. Ownership information is incorporated into type expressions, akin to the Flexible Alias Protection. That is, ownership types are types annotated with context declarations [21, 52, 62]. The ownership relationships between objects can be thought of as a tree. All objects in a system must be brought together into a single ownership tree [62]. This way, owned objects can be protected from being accessed directly from the outside of the owner's context. Any object outside a context must go through the owner in order to have access to the other objects inside the context.

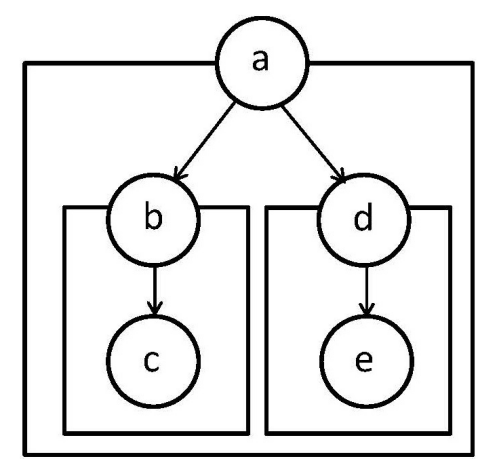

Figure 2.4: Ownership Tree with Sharing Contexts

Consider the ownership tree, depicted in Fig. 2.4, object a owns objects $\mathrm{b}$ and $\mathrm{d}$, and hence the three of them form one sharing context. Objects $\mathrm{b}$ and $\mathrm{c}$ form another context, and hence $\mathrm{a}$ and $\mathrm{d}$ cannot access $\mathrm{c}$ unless through $\mathrm{b}$. Consider the path from $\mathrm{c}$ to $e$, or from $e$ to $c$, objects $\mathrm{c}$ and $\mathrm{e}$ can access directly any object in that path as long as they do not penetrate context boundaries. That is, $c$ can access directly any object but not $e$, and e can access directly any object but not $c$. 
The most researched two approaches to object ownership are: ownersas-dominators [62] and owners-as-modifiers [25].

The owners-as-dominators approach stresses that the dominator object (the owner) is the only entry point for accessing the objects it owns. No one single reference can bypass the dominator. An object can only be referenced by references that do pass through its owner. In other words, the path from the root to the private internal representation of any object must pass through the given object.

The owners-as-modifiers approach implies that an object can only be modified by references that do pass through the owner of that object, but it can be referenced by any arbitrary object with no restrictions. That is, read-only references are allowed to bypass the owner, but cannot update fields or invoke methods.

In the next two subsections, two object ownership models will be described. The first model, deep ownership [19, 21], adopts the owners-asdominators approach. The second model, universes [45, 46], adopts the owners-as-modifiers approach.

\subsubsection{Deep Ownership}

Paradigms that adopt a fully nested scheme of the owners-as-dominators approach are called deep ownership types [19, 21]. The notion of object contexts accommodates the capability to organize the heap into nested constructs. Consider the ownership tree, depicted in Fig. 2.5, we say that the root context is World. That is, the root context is the set of objects owned by world. Objects which are owned by World are accessible to all objects. The solid arrows between objects represent the ownership relationships. The boxes represent the context boundaries. The dashed arrows represent the references that should stop by the boundaries and pass through the right entry point (i.e., the owner). 
Still with Fig. 2.5, the ownership relationships can be seen as a tree rooted at World. An owner is another object or World. Every object should have an owner. An object can have only one owner. The owner cannot be changed during the lifespan of the object. Object a owns b but does not own c. An object can access the objects it owns. An object can access its ancestors and objects they own. That is, any object can access any object owned by World. Henceforth, the notion of containment [21] (inside-outside relationships) can be used as we describe the relationships between objects. That is, if we say that $b$ and $d$ are inside $a$, and $c$ is inside $b$, then this means that $b$ and $d$ are owned by $a$ or in $a$ 's context, and $c$ is owned by $b$ or in b's context. Furthermore, we say that $b$ and $d$ are siblings.

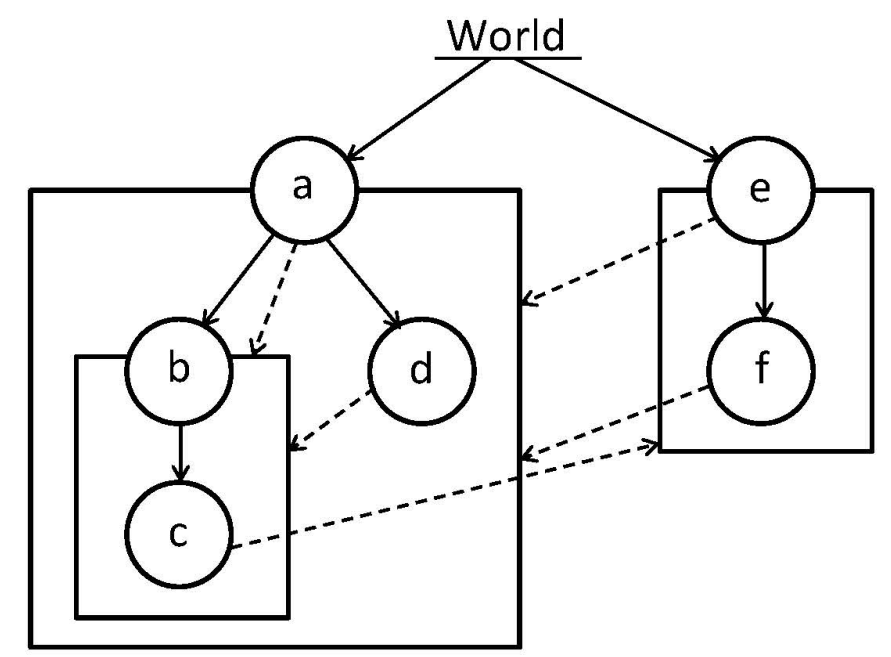

Figure 2.5: Deep Ownership Relationships Between Objects.

The earliest ownership type system [21] introduces ownership types as static types annotated with context declarations. The rep annotation denotes an object as owned by this; that is, a rep object is part of the owner's private representation. The Owner annotation denotes an object as having the same owner as this; that is, a sibling object to the enclosing this. The World annotation denotes the absence of an identified owner; 
that is, objects owned by World can own but cannot be owned; visible to, and accessable by, all objects but not dominated.

This model does not permit a container's iterator to access the container's internal representation. This problem is tackled by permitting inner classes [9] or local variables [17] to have special privileges, or by way of using unique incoming references [19]. The idea is that deep ownership imposes an equal treatment of inner classes and their enclosing classes. That is, inner classes' instances cannot share the internal representation of their enclosing object. Boyapati et al. [9] considered this restriction too strict to support constructs like iterators; and thus, argue that the appropriate way to relax the owners-as-dominators property is to permit inner classes' instances and their enclosing object to have a common representation. A comprehensive study on the use of ownership types in design patterns [50] concludes that permitting an inner class to have privileged access to its enclosing class's internal representation has not shown very useful apart from the iterator pattern; and that even with highly collaborating classes, the use of inner classes is undue in most situations.

Since an iterator needs to be able to access the elements stored in its respective aggregate, the typical approach taken for implementing iterators is to declare them as inner classes. Consider the diagram in Fig,2.6, deep ownership requires an aggregate object to own its private representation. That is, the representation is directly inside the owner context. Since an iterator is usually instantiated independently of its enclosing aggregate, then it should not be able to access the aggregate's representation without the aggregate's knowledge. That is, an iterator should pass through its aggregate's operations in order to access the private representation. The proposed ad hoc relaxation, by Boyapati et al. [9], critically violates the representation containment invariance [21] necessary for deep ownership types, and breaks encapsulation, since incoming aliases are allowed to instances of iterators that share the internal representation of their enclosing aggregates. 


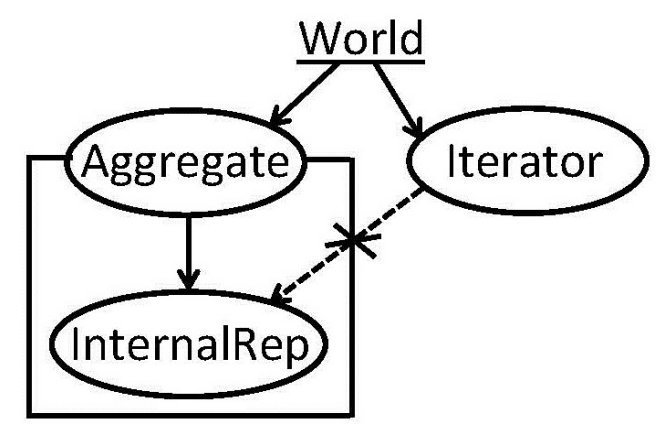

Figure 2.6: References must not cross a context boundary from the outside to the inside

\subsubsection{Universes}

Müller and Poetzsch-Heffter [45, 46] introduced Universes with the following aspirations: Universes needs to (1) have simple semantics, (2) be easy to apply, (3) be statically checkable (4) guarantee an invariant that is strong enough for modular reasoning, and (5) be flexible enough for many useful programming patterns. The Universe type system is an ownership type system to enforce the owner-as-modifier approach that has been inspired by Flexible Alias Protection. The original syntax of Universes, which is different from the current one, used a type combinator, higher-order function, to construct types. Later in 2001, Müller and Poetzsch-Heffter [47] used three ownership modifiers peer, rep, and readonly (changed later to be any), to extend all type declarations with one of these modifiers. The modifier peer denotes an object as having the same owner as this; rep denotes an object as owned by this; and readonly denotes an object as capable of having any owner, but readon 1 y references cannot update fields or invoke methods. The type combinator function takes two of these modifiers (both arguments can be the same modifier) and returns the resulting ownership modifier to determine the type of field accesses and method call parameters and results. That is, the ownership type of $x . f$ is determined, according to the combination to be made inside the type 
combinator, by passing the ownership modifiers of both $\mathrm{x}$ and $\mathrm{f}$ as reference parameters. The table in Fig. 2.7 is used to determine the resulting modifier. The table uses modifier this, which cannot be supplied by a user, as the modifier of receiver this.

\begin{tabular}{l|lll} 
& readonly & peer & rep \\
\hline this & readonly & peer & rep \\
readonly & readonly & readonly & readonly \\
peer & readonly & peer & readonly \\
rep & readonly & rep & readonly
\end{tabular}

Figure 2.7: Universe Types Modifier Combinator.

The Universe type system has achieved gradual development in the past years. The most notable ones: are the integration into the Java Modeling Language (JML) [25], and another type system called Generic Universe Types (GUT) [23] to integrate the owner-as-modifier property into generic types in mainstream object-oriented languages; GUT was also implemented in JML.

The use of read-only references, that can have any owner, makes some programming idioms more expressible with this model than with the deep ownership model. Nevertheless, deep ownership and universes have been evaluated [50] for compatibility with object-oriented design patterns, and have not proved sufficient. The evaluation suggests that many design patterns require moving particular objects, after they have been initialised, from their originating contexts to some other contexts.

\subsubsection{External Uniqueness}

In conjunction with deep ownership types, a relaxed form of uniqueness called External Uniqueness [19] validates the use of incoming references only if they are constrained to be unique. 
As explained in subsection 2.2.3, uniqueness is not practical for aggregate objects, since a unique reference cannot refer to the internally aliased components. Clarke and Wrigstad [19] propose that a component object can have only one alias from the outside of its aggregate, while still being aliased freely from the inside of the aggregate. That is, internal aliases to an externally unique object cannot be observed by external clients.

In conjunction with deep ownership, this proposal suggests that an externally unique object can be safely transferred from one context to another sibling context, as depicted in Fig. 2.8. Clarke and Wrigstad argue that this form of ownership transfer is sufficient for concurrent object-oriented programming.

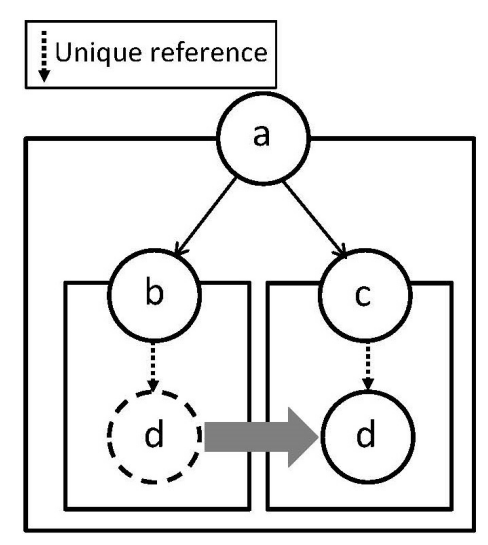

Figure 2.8: Ownership Transfer of an Externally Unique Object

\subsubsection{Ownership Generic Java (OGJ)}

As a combined mechanism to facilitate deep ownership types in conjunction with parametric polymorphism, Potanin et.al. [59, 60, 61] introduced Generic Ownership along with an extended version of Java, named Ownership Generic Java (OGJ). This combination provides the ability to constrain aliasing and detect errors statically, while neither demands an added syntactic extension nor imposes overheads at runtime. This is because OGJ 
treats ownership information as supplementary generic types. That is, Java Generics are extended by the ability to hold, and interpret, ownership information.

In OGJ, any class declaration needs to allocate at least one type parameter to receive context declarations as type arguments. Usually, this type parameter is bounded by World, in which case it is dubbed owner parameter. A possible declaration would read as:

$$
\text { class Foo<Owner extends World }>\{\ldots\}
$$

If an instantiation such as new Bar<Owner> () is created within Foo's class body, then Foo and Bar are siblings owned by the same owner. OGJ uses the last type parameter to denote an object's owner. For example, instantiating a List container can read as:

List<String, Owner> 1 = new ArrayList<String, Owner>();

OGJ introduced three ownership domains [3], namely World, This, and Package. Objects owned by World are global objects that can be freely aliased by any arbitrary object. Objects owned by This can only be aliased within the class in which they have been created; they can only be accessed through this as a receiver. That is, owner argument This denotes an object as owned by an instance of the enclosing class. With regard to the ownership tree which is depicted in Fig. 2.5, there seems no need for more context declarations in order to maintain deep ownership types. We have global objects, private hidden objects, and sibling objects which are owner parameterised by the same owner variable of the enclosing class. In order to make some programming idioms more expressible, the ownership domain Package is introduced in OGJ so that objects owned by Package can only be aliased local to the package in which they have been created, with no restrictions on how they can be aliased within the package. This follows from the Confined Types proposal [64]. 


\subsubsection{Wildcards and Generic Ownership}

WOGJ [13] and Jo $\exists$ [12] applies the theory on existential types [56] to generic ownership. Both systems provide theoretical foundations for employing Java wildcards in favour of ownership types. The fundamental goal of both systems is to eliminate the special treatment of the This context in parametric ownership type systems, so that the heap structure becomes independent of the encapsulation system, and thus there is no enforcement of the owners-as-dominators property. $\mathrm{Jo}_{\text {deep }}$ makes this enforcement optional. The above mentioned systems are proved to be theoretically sound, with no practical language design or implementation.

\subsubsection{Generic Universe Types (GUT)}

As is the case with OGJ, Dietl et.al. [23] combined Universe Types with type genericity. GUT is integrated into JML. GUT enforces the owneras-modifier approach. As mentioned above, this approach provides for an object to only be modified through its owner, and does not limit the freedom to alias. What applies to the original universe types applies to the generic universe types: the ownership information is object-specific, not class-specific; it provides static control; and annotation overheads.

As is the case with the universe type system, the type combinator function is relied on. The idea is that the three ownership modifiers peer, rep, and any were remodelled to couple type arguments. A possible declaration would be as:

rep Iter<any Node<rep ID, any Data>> i;

That is, the ownership information is not type parameters, but instead is associated with type arguments as well as with type instances (parameterized and non-parameterized). 


\section{Chapter 3}

\section{OG $\mathbf{J}_{+}$Language}

This chapter explains the main concepts of $O G J_{+}$, our extension of Java 6 with support for deep ownership types. OGJ provides support for generic ownership types, and addresses all of the remaining open issues that were not addressed in OGJ [57, 59, 60, 61]. That is, OGJ treats arrays, inner classes, static contexts, wildcard types, clone, equals, enum types and exception handling. We allocate the next chapter to these open issues.

The previous version of OGJ is an extension of Java 5 and provides ownership support for the basic features such as classes and subtyping, field access and assignment, and methods. $\mathrm{OGJ}_{+}$treats these basic features in a broader perspective, so that interfaces, constructors, method parameters, return types, method invocations and casts can conform to our treatment of the other features. Ideas will be clarified gradually as we progress with this chapter and the next chapter. Altogether, $\mathrm{OGJ}_{+}$is ownership for all of Java.

$\mathrm{OGJ}_{+}$provides a very restrictive form of encapsulation. The basis of this is a strict adherence to the rules of the deep ownership encapsulation model which is too strict to support some programming idioms and design patterns. The resulting language is rather verbose. Existing Java programs might require significant restructuring in order to comply with $\mathrm{OGJ}_{+}$. 


\subsection{An OGJ $J_{+}$Example}

Although the very basic concepts of generic ownership are provided in the previous chapter, we will re-explain them using the example in Fig. 3.1. which is an $\mathrm{OGJ}_{+}$compliant version of the example in Fig. 2.2.

An OGJ + program may involve only two ownership domains: World and This. The first type parameter in a generic parameter list is always reserved to denote an object's owner.

Class instances owned by World (see line 24, Fig. 3.1) are public objects, to which access is unrestricted and they are visible to all objects in the ownership tree. That is, World-owned objects belong to the root context.

Objects owned by This (see line 11) are private objects that need to remain hidden from the other objects in the tree, except from their enclosing objects. That is, This-owned objects belong to the current object, and can only be referred to by this. The use of owner This is exemplified by class Widget. Widget objects own their Point objects as they form the internal representation. This dominance is represented by owner This of Widget's field wPos, which refers to the widget's position.

Finally, there are objects that need to have the same owner as the this object. A referenced object can share the same owner as the current object in order to share the same context. In line 17, the local variable pos is owner parameterised by Owner, which is the same owner variable used to specify the owner of the enclosing class Widget (see line 10). That is, any pos object will always be owned by the same owner object as the enclosing $\mathrm{W}$ idget instance.

The class headers at lines 1, 10 and 21 illustrate how class owners can be defined. Since Owner is bounded by World, a class can be instantiated as (1) a public object owned by World (see line 24); (2) a private object owned by This (see line 11), since This is a subtype of World; or (3) a sibling object owned by Owner (see lines 13 \& 17). 


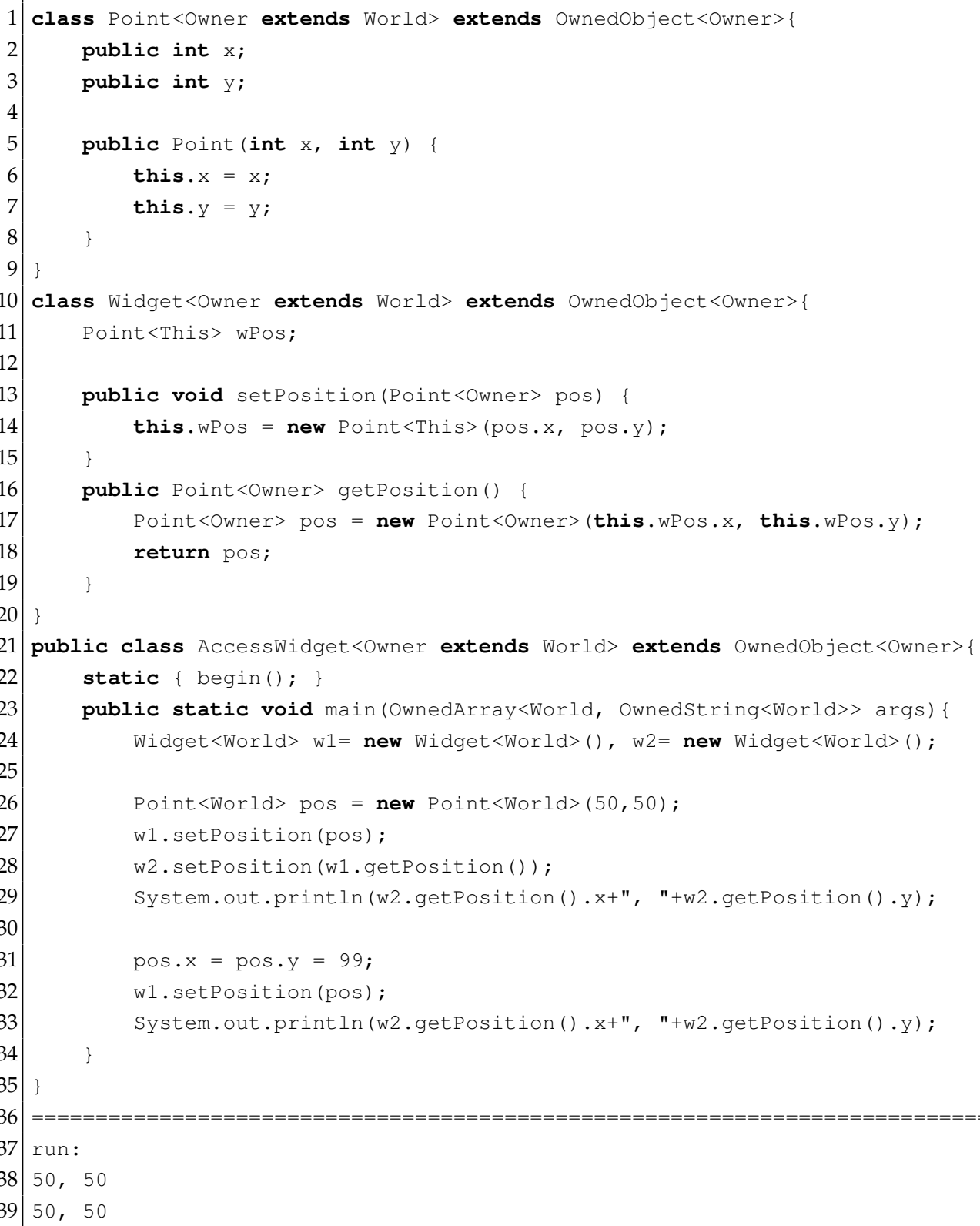

Figure 3.1: A generic ownership compliant version of Fig. 2.2. 
Methods, whose formal parameter types and/or return types are owned by This, can also only be invoked within the current object (i.e., as accessible via this) because owner This is instance-specific and is not compatible with owner This in another class. As a result, we could not have Point<This> as a parameter type and return type in setPosition() and getPosition(), respectively, since invoking these methods in another class, the public class (line 21), is no more possible than through owners other than This. Since Widget's field wPos is owned by This, it is not possible within setPosition () to assign the Owner-owned parameter Pos to this.wPos; owners are incompatible. The same applies to getPosition(), it is not possible to return this.wPos. In this example, the chosen solution is to use value semantics instead of reference semantics; that is, getPosition() clones the return value, and setPosition() clones the actual parameter's value. Value semantics are not inevitable in $\mathrm{OGJ}_{+}$, as will be discussed later in this chapter.

\subsection{Reference Types}

There are two kinds of reference types: type variables and nonvariable types. A type variable in $O G J_{+}$needs to have an explicitly owned bound, as illustrated by type variable $\mathrm{T}$ in Fig. 3.2; the example shows a possible formation of a class header in $\mathrm{OGJ}_{+}$. As illustrated by the example, $\mathrm{T}$ is bounded by Ownedobject, which is the root of the class hierarchy in $\mathrm{OGJ}_{+}$, and is owner parameterised by the owner variable TOwner.

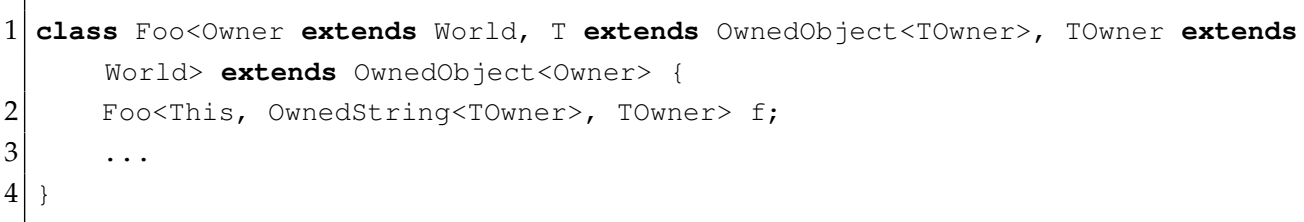

Figure 3.2: A possible $O G J_{+}$class header. 
A nonvariable type in $O G J_{+}$involves a class name, owner parameter, and optional type arguments. Type Foo in line 2, Fig. 3.2, is an example of a nonvariable type; Foo involves the type argument OwnedString plus two owners, one as the first parameter and the other as the last parameter in the generic parameter list. $O G J_{+}$uses the first type parameter to denote an object's owner. Such an owner is conventionally known as the distinguished owner, and is mandatory since every object should be owned. The distinguished owner must be preserved over subtyping, as will be described in subsection 3.3.1. Consider the header of class Foo, there are two different owner variables defined, Owner and TOwner. Owner is Foo's distinguished owner, and any owner argument in place of Owner will denote the current Foo object's owner. As for TOwner, this is what we call a normal owner parameter. Normal owners can occur, more than once, anywhere in the generic parameter list. In this example, we use TOwner to constrain the owner of the generic type argument of $\mathrm{T}$.

There are times when owner parameters are not just required to define distinguished owners or to constrain the owners of type parameters, but for example to infer the objects' owners. Consider the example in Fig. 3.3 . Foo1 owns $f 2$ as represented by owner This in line 2. Java will not allow the method invocation, in line 4 , if any of the owners World, This, Owner2 occurs in place of MOwner (line 6). Java does not allow this because the type parameter (or owner parameter in $O G J_{+}$'s parlance) should match the owner of this, which is Owner1. For methods in Java to facil-

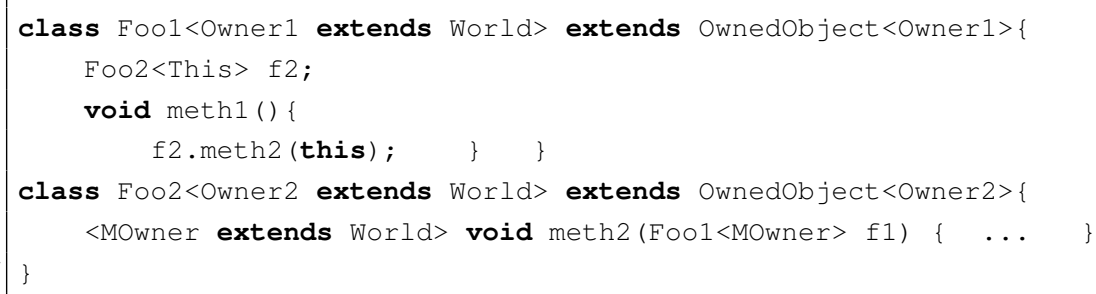

Figure 3.3: Using a naked owner to infer ownership information. 
itate type argument inference [28], they had to be generified. That is, the generic parametricity of method meth2 (), in line 6, allows the method to get the correct owner of this as a result of the invocation in line 4 . In comparison to normal owners, the owner parameter in this method's signature does not constrain a type parameter's owner. Such an owner is what we call a naked owner parameter.

Finally, $O G J_{+}$provides the possibility to define a placeholder corresponding to a yet to be defined representation context, through the use of wildcards. The example in Fig. 3.4 shows the header of class OwnedArray, which we use in OGJ to wrap array objects, as will be explained in subsection 4.1 . The formal type parameter $\mathrm{T}$ is bounded by Ownedobject, but the owner (representation context) is not explicitly provided as we use a wildcard in place of the actual owner parameter. As will be explained in subsection 3.6, a wildcard owner parameter must be bounded by World. Line 23 in Fig. 3.1 shows how type OwnedArray involves the type argument OwnedString as being located in the root context.

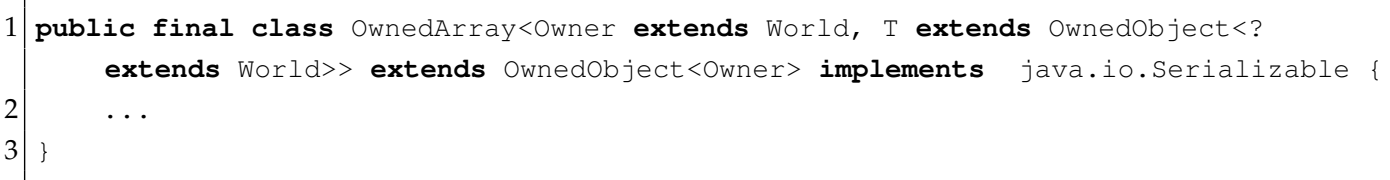

Figure 3.4: OwnedArray class header.

\subsection{Class Hierarchy}

In deep ownership, the root of the class hierarchy can be envisioned as a context-specific root. That is, the root of one context is not the same as the root of another, since the heap is transformed into separate nested parts, where Ob ject cannot jump arbitrarily from one to another. Strictly speaking, each context should be able to have its own object, which means that Object should also be type-parameterised by an owner parameter, 
as is the case with every other class. To facilitate this, $O G J_{+}$provides a new class called Ownedobject<Owner> to serve as the root class in its respective context; and thus, subtyping Object is no longer permitted. Accordingly, a typical $O G J_{+}$class declaration would be as in line 1 of Fig. 3.5 .

\subsubsection{Subtyping}

Context information must be preserved over subtyping [18]. That is, we need to map the owner of the subclass to the owner of the superclass, as appears in lines 1 and 7, Fig. 3.5. This is enforced by the type checker; otherwise, objects can overstep context boundaries using subtyping. Consider the class declaration in line 3, Fig. 3.5, where the owner of Bar is not mapped to the owner of Foo. As a consequence of this class declaration, Java allows the assignment statement in line 10, where a public object from the root context is able to refer to a private object that is supposed to be hidden in a nested context.

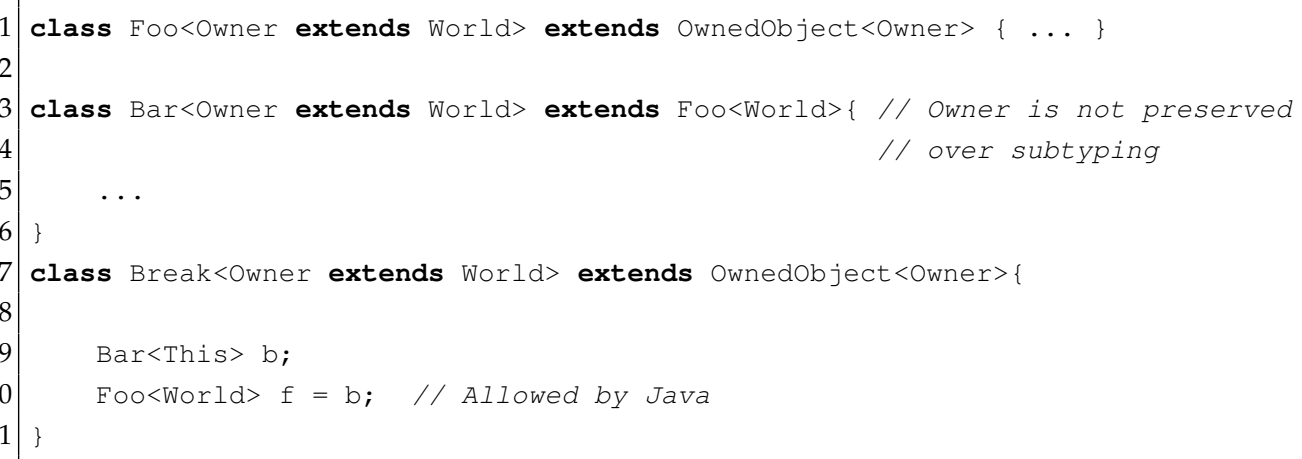

Figure 3.5: Context information must be preserved.

By preserving ownership over subtyping, the nesting among contexts is preserved and objects cannot overstep the boundary limitations, imposed by the dominators property, using subtyping. Assignment operations should always involve compatible owners. An object's value is 
assignable to a reference variable of a supertype of the given object, only if they share the same ownership context. Since each ownership context must have its own class hierarchy, the context information that is propagated amongst the objects contained in one context is not compatible in another context. That is, two instances of the same type, or supertype, are considered incompatible (not assignable to each other) if they have different owners.

\subsubsection{Interfaces}

Now that extending Object is prohibited, and every single object is enforced to be owned and to only subtype owned objects; we still have interfaces: Java expects every interface to only extend another interface. To overcome this, an interface called IOwnedObject $<$ Owner $>$ is implemented so that an interface can have its root originated from within its respective context. Similar to class declarations, an interface must preserve its owner over subtyping.

Any class in $\mathrm{OGJ}_{+}$must be a descendant of Ownedobject; and any interface must be a descendant of IOwnedobject. By design, OwnedObject implements IOwnedObject, and preserves its own owner over this subtyping. Since the owner of a class must be preserved over subtyping, interfaces are safe from being inherited (using implements) with a different owner than that of the given class. 


\subsection{Nesting Scheme}

As explained in chapter 2, deep ownership is a fully nested scheme of the owners-as-dominators approach to encapsulation. The owner nesting type checking can be concisely explained as going over every individual owner parameter involved in any generic parameter list to verify if it is the same or outside the distinguished owner of the defining object. For domain parameters, this means that This is inside everything, and World is outside everything. That is, if the distinguished owner is This, then there will be no restrictions placed on the other owner parameters involved; if the distinguished owner is World, then all of the other owner parameters should be World. For owner variables, the relationships between them will be clarified as we describe type checking against variable definitions in subsection 3.4.2. Owner nesting needs to be verified for every class, method and field/variable declaration. The rest of this subsection describes how the inside-outside relationships can be maintained, and what happens if these relationships are broken.

\subsubsection{Class Declaration}

The determination of nesting relationships amongst the owner parameters in a class header can be seen as if it is amongst the bounds of these owner parameters; and if the owner bound is itself an owner variable, then the compiler will go find its bound, and so on. As in most cases, if the distinguished owner bound is World, then every other bound should be World.

\subsubsection{Field/Variable Declaration}

All kinds of variables (namely fields, local variables and parameters) are treated the same, when it comes to owner nesting verification within the types of the variables. OGJ + assumes that This is inside everything, World is outside everything, and the distinguished owner variable of the 
enclosing class is inside every other owner variable involved in the header of that enclosing class. This means that the field declaration in line 4 of Fig. 3.6 is illegal, since Owner 1 is inside Owner2. If this is not illegal, then the instantiation in line 13 will result in InnerFoo<World, This $>f$; which breaks the nesting rule, and exposes the field to the outside world, while the defining object (i.e., the current FoO) is supposed to be hidden, and be able to hide its interior as need be.

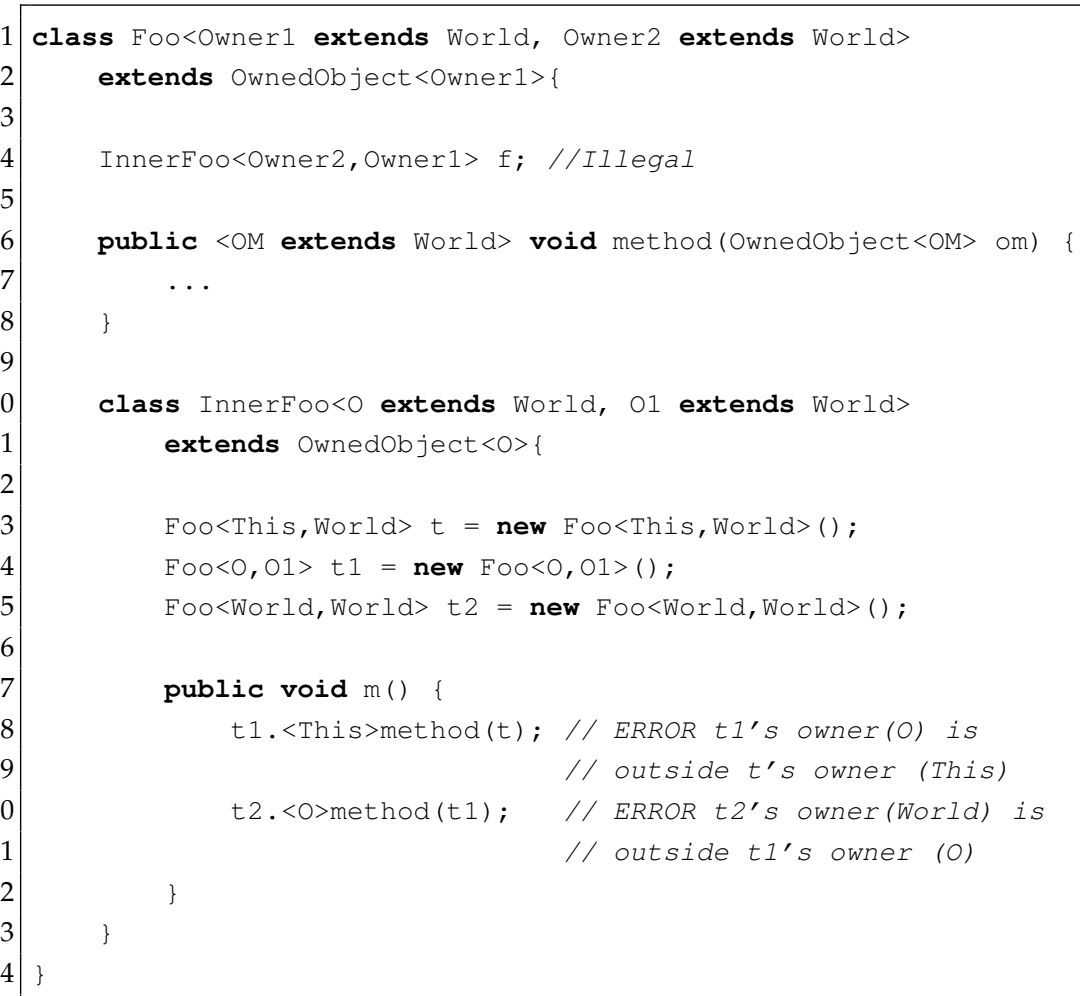

Figure 3.6: Illegal Owner Nesting.

\subsubsection{Method Declaration}

In certain cases, such as the one described in subsection 3.2, a method declaration might need to be owner parameterised with a supplementary 
naked owner parameter to infer the correct owner when the method is being invoked in different ownership contexts. In such a case, this owner parameter will need to be checked for owner nesting against the distinguished owner of the enclosing class. Correspondingly, when it comes to method invocation, the owner of the method receiver must be inside every actual owner parameter used as a distinguished owner for any of the method's arguments. Consider the erroneous method invocations in lines 18 and 20 of Fig. 3.6. The relationship between the objects involved in the first method invocation is depicted in Fig. 3.7. The owner parameter 0 is declared, in line 10, as the owner of InnerFoo. The method receiver t 1 is declared, in line 14, as a sibling to InnerFoo; which means that InnerFoo and $t 1$ are located in $O$ 's ownership context. The method argument $t$ is declared, in line 13, as owned by InnerFoo; which means that $t$ is located in InnerFoo's nesting context. Since $t 1$ is located outside InnerFoo's context, $t$ in not allowed to cross InnerFoo's context boundary and access $t$. That is why we have to ensure that the owner of the receiver is inside the owner of the argument. The same idea applies to the method invocation in line 20; $t 2$ is owned by World which is outside everything; that is, the owner of the argument cannot be anything other than world.

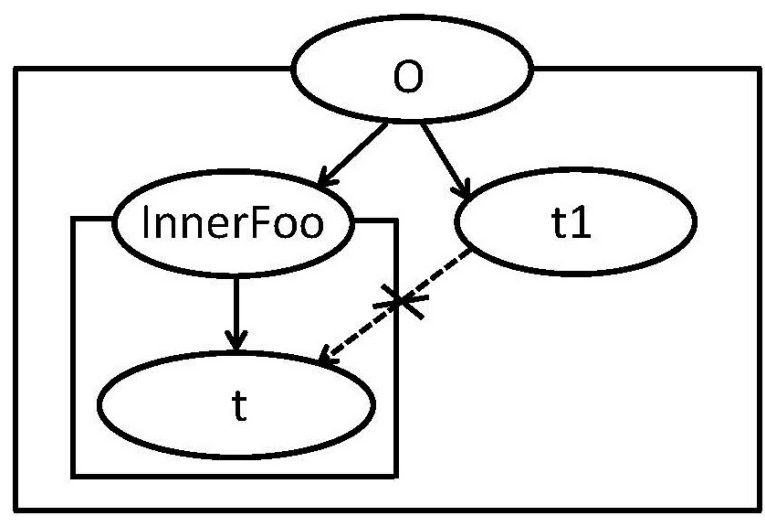

Figure 3.7: Context boundaries should not be broken through method invocations. 


\subsection{Instantiation and Casting}

Ownership information should not be lost while instantiating a new object or due to casting to a particular type. Type erasure is the process by which the Java compiler removes all type parametricity information, parameters as well as arguments, by replacing all type variables with only the class names of their bounds, or with Object if a type variable is not bounded. On that account, programmers are allowed to drop the actual type parameters from parameterised types as need be. A parameterised type used without an accompanying type argument is conventionally known a raw type. For $\mathrm{OGJ}_{+}$to ensure that ownership information cannot be lost, raw types are forbidden in all respects. Consider the different kinds of erroneous statements in Fig. 3.8. In addition to the prevention of casting to raw types, and object, casts such as the ones shown in lines 9 and 10 should not be allowed as they create a sharable reference to a private object, in the first case; and a public reference to a within-context sharable object, in the second case. That is, cast ownership information should be the same as the instance ownership information.

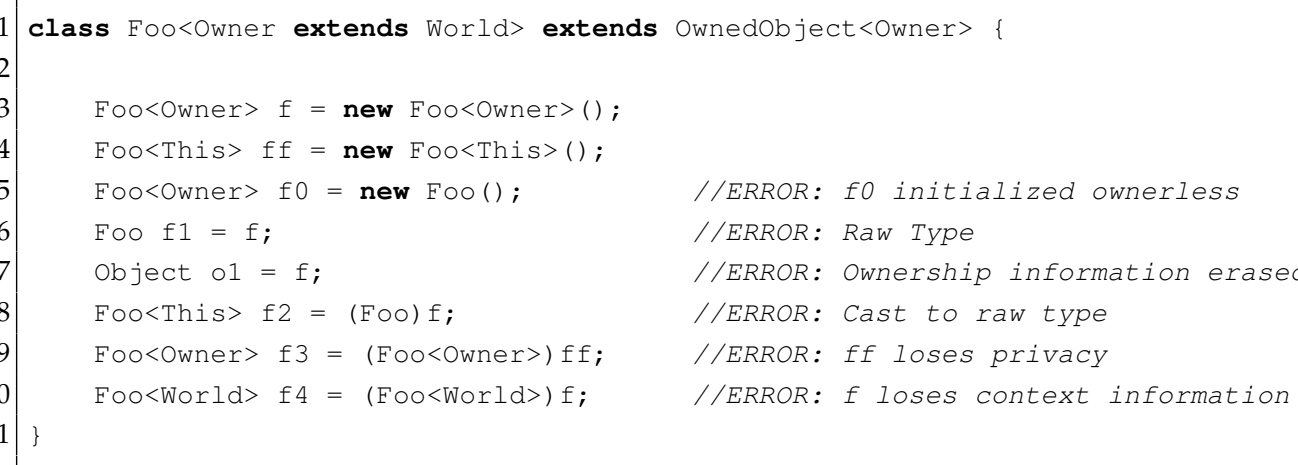

Figure 3.8: Preserving Ownership Information

Upcasting happens implicitly whenever a reference to a subtype is assigned to a supertype reference, and the supertype might not involve the type parameters that its subtypes might involve, as illustrated in lines 3 
and 9, Fig . 3.9. Downcasting the supertype reference as in line 10 is allowed by Java, but will result in $f 2$ referring to the same object as $f 1$ with different ownership information, since the actual type parameter FO०1 of $\mathrm{f} 2$ has different ownership information than that of $f 1$ 's Fool. OGJ hibits the kind of downcast in line 10, but unfortunately prohibits also the downcast in line 11, since it does not provide a mechanism for preserving the ownership information of runtime downcasts [8]. Nevertheless, OGJ mitigates this restriction through the use of wildcard owner parameters, as illustrated in lines 6 and 12. The safety of utilising the wildcard feature of Java generics will be described in detail in the next section.

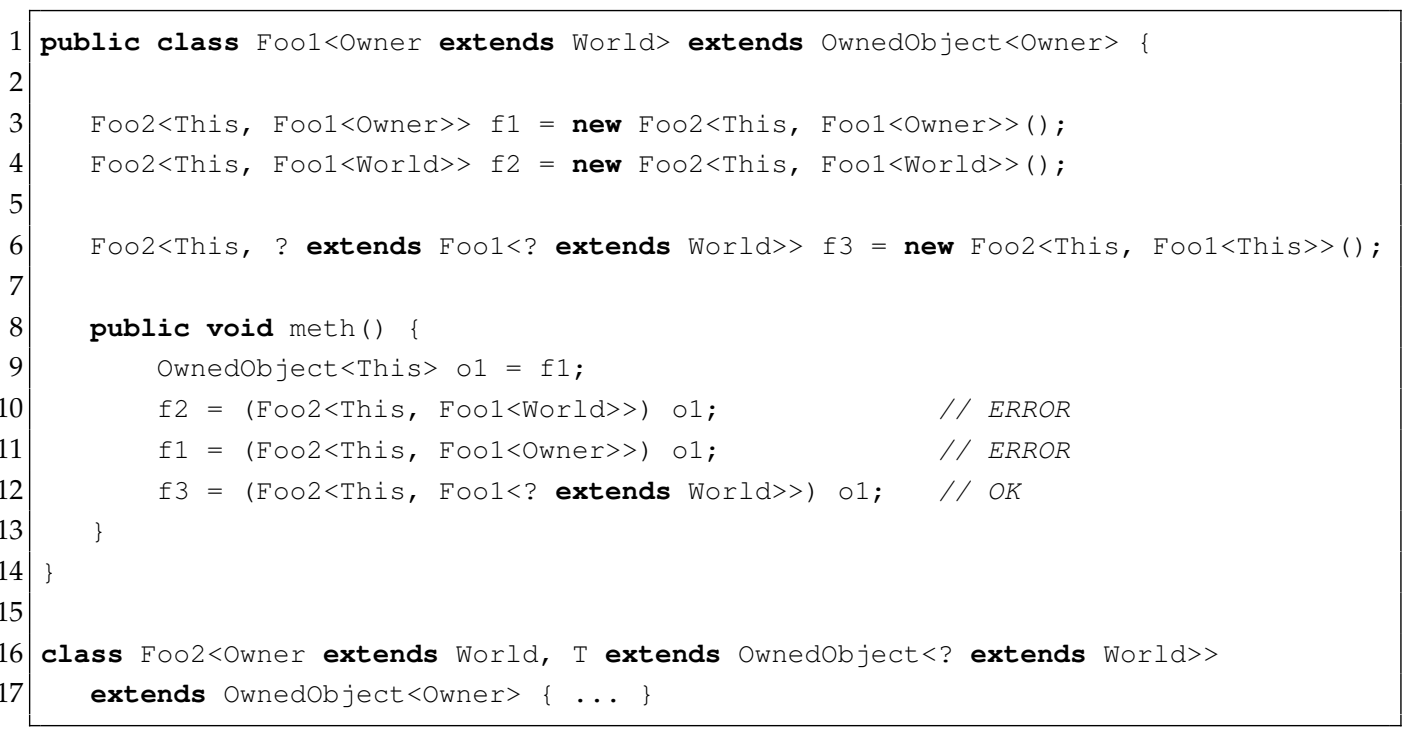

Figure 3.9: Preserving Ownership Information against Up/DownCasting

The idea of using wildcards to synthesise owner parameters [15], when downcasting from one type to another, while the latter has ownership information that the former has not, takes its root from "Existential Downcasting" proposed by Wrigstad and Clarke [68]. The authors simplify the idea as "if a Java-style downcast (disregarding ownership) of an object to some class $c$ succeeds, then we could infer the owner parameters necessary to form the new type from c's class header. We call the inferred owners existential owners, and 
types that use them existential types". Wrigstad and Clarke built their case based on the Java equals idiom, and argue that it is not sufficient for the overriding equals () operations to keep track of the owners at run-time by extending each class by a field for each owner the class uses [8]. The equals idiom will be described in section 4.5 .

\subsection{Wildcard Types}

Generic types should be invariant in sound type systems; therefore, Java does not accommodate a built-in conversion from, for example, List<Manager $>$ to List<Employee $>$ based on the datum that Manager is a subtype of Employee. That is, there should be no dependency, or subtype covariance, between two objects generated from a single generic class; otherwise, employees who are not managers might be added to List<Manager>. As a consequence, it is not possible to write a reusable subroutine that can list the different categories of employees by parameterising the subroutine with List $\langle$ Employee $>$, or even List<Object>, because Java generics are not associated with a builtin subtype covariance. Nevertheless, to attain the flexibility required for writing reusable software, Java facilitates a programmer-defined covariant subtyping through wildcard types [28].

A wildcard type is a generic type that uses ? as a type argument, for example List<?>. Wildcard types are carefully integrated with Java generics to provide as safe as possible form of covariant subtyping. For example, Java does not allow any add operations to a list object of type List $\langle$ ?>, since the element type of the list object is unknown, and thus the creation of new element objects is disallowed. In contrast, retrieve operations can face less restriction in making use of the result, since a result type is, in the end, of type object. Nevertheless, a wildcard type can be further constrained as List<? extends Employee>, which means that the unknown element type must be a subtype of 
Employee; and we say that Employee is the upper bound of the wildcard. An unbounded wildcard type, such as List<?>, has the same interpretation as List<? extends object>. It is also possible to define a lower bound for the wildcard using the keyword super; for example, List<? super Trainee>, which means that the unknown element type must be a supertype of Trainee. Furthermore, the bounding types can be parameterised types; and here is exactly how OGJ + can support wildcard types, since all reference types must be owner parameterised in $O G J_{+}$.

$\mathrm{OGJ}_{+}$provides the same programmer-defined covariant subtyping mechanism as Java. While Collection<? $>$ is the supertype of all kinds of Java collections, Collection $\langle 0$, ? extends OwnedObject $\langle E O\rangle$, EO $\rangle$ is a possible supertype of all kinds of collections located in the same ownership context, where $O$ is the Collection's distinguished owner, and $\mathrm{EO}$ is the owner of the unknown element type. The use of unbounded wildcard types in $O G J_{+}$is not allowed, since object is no longer the root class; a wildcard should be bounded by 'Ownedob ject', 'IOwnedobject', or a class that is subtyping any of them.

\subsubsection{Ownership Context Covariance}

$\mathrm{OGJ}_{+}$also supports ownership context covariance. That is, an object can belong to an unknown ownership context for more flexible code reuse. A wildcard can occur in place of an actual owner parameter, but can only be bounded by world. Accordingly, the supertype of all kinds of collections in a yet to be defined context is as follows:

Collection<? extends World,

? extends OwnedObject $<$ ? extends World $>>$

If a distinguished owner is a wildcard, then any other owner argument must be either a wildcard or World. This is because if we have, for example, a field, local variable, or method parameter of type 
List<? extends World,...>, we do not know which owner would replace the wildcard. The owner might be Wor $1 d$, which means that every other owner must be World in order to preserve the nesting relationships between owners - the distinguished owner must be inside every other owner, and World is outside everything. So, having a wildcard as the distinguished owner necessitates that all of the other actual owner parameters be made nesting Wor ld via a wildcard, or Wor $1 \mathrm{~d}$ as infrequently need be. Declaring an object, whose type is a wildcard owner parameterised, is subject to owner nesting verification by treating every wildcard as Wor ld. This way, it is possible to have a non-wildcard distinguished owner (e.g., World, This, or an owner variable), while wildcards can be used in place of other owner arguments. Since every declared object (with wildcards or without) is subject to owner nesting verification, the nesting scheme is not to be violated. The example in Fig. 3.10 illustrates the use of wildcard types in $\mathrm{OGJ}_{+}$. Method printList () is able to access list objects of unknown contexts. 1 ist 01 and list 02 (declared in lines 16 and 21, respectively) are objects located in different contexts. Passing 1 ist 01 and list02 to printList () (lines 26 and 27) is safe since the type checking required for owner nesting verification is applied beforehand at the declaration sites. 


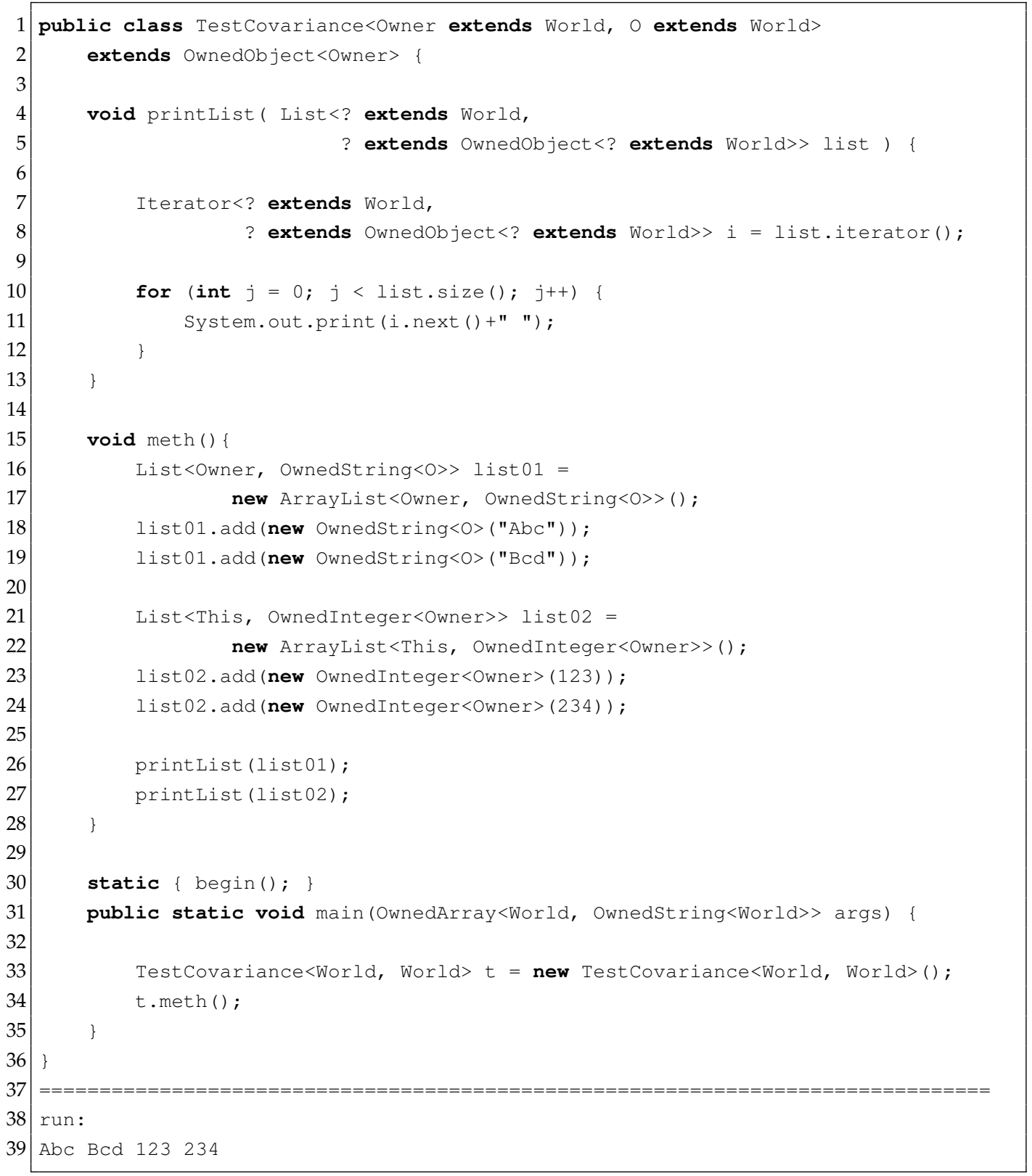

Figure 3.10: Generic Ownership Wildcard Types 
As is clarified in previous subsections, the meaning of owner This relies on the presence of receiver this. Accordingly, invoking methods, which involve owner This within their formal parameter types and/or return types, must be via receiver this. Nevertheless, a method might involve owner This at the time of invocation, not at the time of declaration, if it is an owner parameterised method (line 2, Fig. 3.11). In such a case, $\mathrm{OGJ}_{+}$will not check if owner This is involved within the declaration signature or not, or if receiver this is involved in the invocation or not, but owner nesting verification will be applied to ensure that the distinguished owner of the receiver is inside every actual owner parameter used as a distinguished owner for any of the method's arguments. So, what if the receiver's owner is a wildcard (lines 12, 13 and 14, Fig. 3.11), or if the method argument's owner is a wildcard (lines 16, 17 and 18)? The only legal method invocation, if the receiver's owner is a wildcard, is that if the method argument's owner is World; and the only legal method invocation, if the method argument's owner is a wildcard, is that if the receiver's owner is This. Otherwise, there is no guarantee that the unknown owner will preserve the nesting relationships.

\subsubsection{Readonly References}

A wildcard owner makes a reference readonly, and hence cannot be manipulated by arbitrary external objects. Consider the example in Fig. 3.12 . class OwnsFoo owns $f 1$ and has a getter method getFoo() that returns $\mathrm{f1}$. The return type of getFoo() is owner parameterised by a wildcard, hence the method can be invoked as in line 14. The other option for this method is to have a return type that is owned by This, in which case the method invocation in line 14 will not be possible in OGJ $J_{+}$. Class TryExpose has the wildcard-owned reference $f 2$ that can be set to any F०० object of any ownership context. The only way, in Java, to write to $f 2$. bar (line 17) is if OwnedString is raw, which cannot be the case 


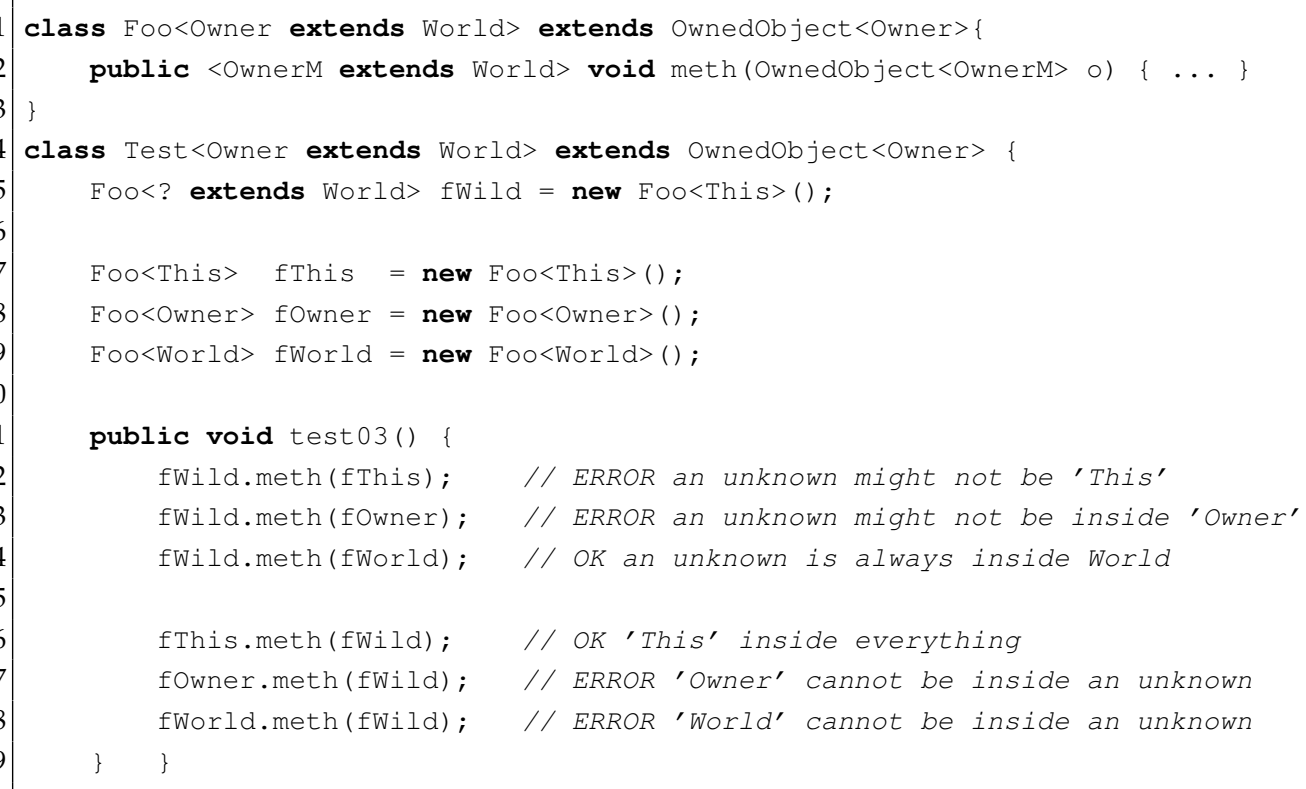

Figure 3.11: Restrictions on method invocations via wildcard owner parameterised receivers

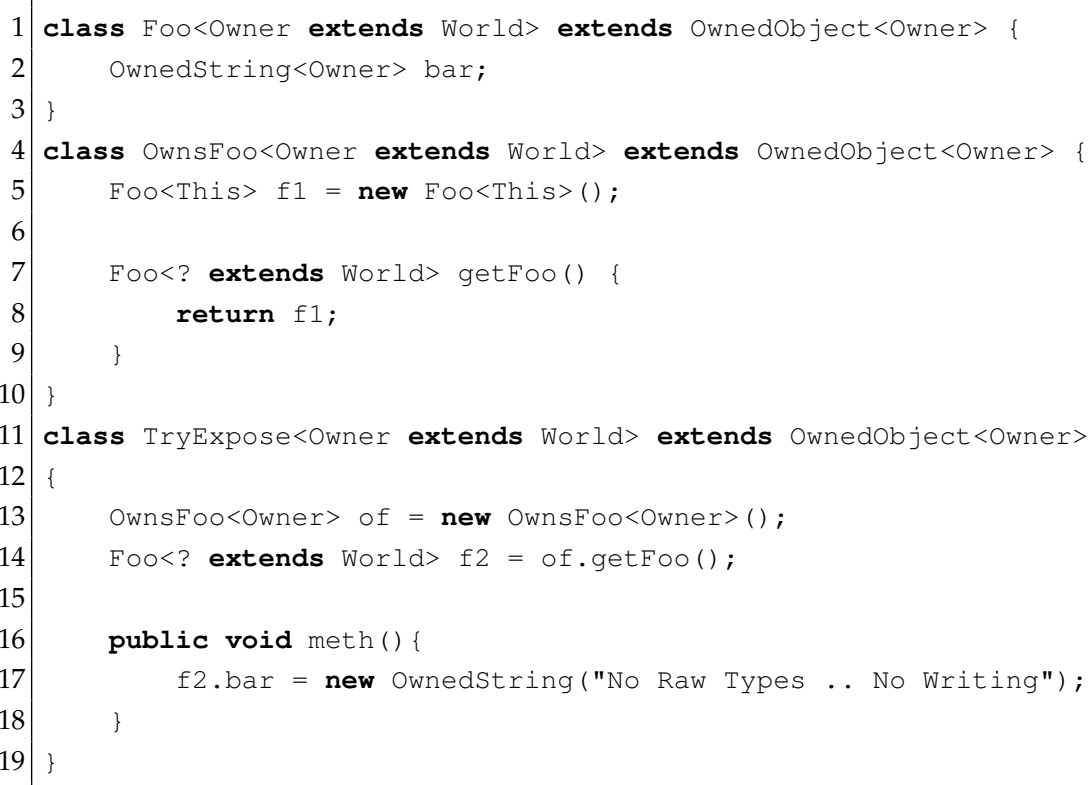

Figure 3.12: Wildcard owned references are readonly 
with $\mathrm{OGJ}_{+}$; and that is why we worry about the ownership of a final immutable object such as String, since all objects in OGJ must belong to an ownership context. Classes in the Java standard library (e.g., Integer, Number, String, etc.) have their corresponding classes in $O G J_{+}$(e.g., OwnedInteger, OwnedNumber, OwnedString, etc.) 


\section{Chapter 4}

\section{From Java To OGJ $\mathbf{J}_{+}$}

\subsection{Arrays}

One of the fundamental issues that any attempt to extend Java with ownership types encounters is arrays. In relation to the use of owners-asdominators, prototype implementations of ownership type systems in the context of Java are usually bound to remain JVM-compatible; very much as is the case with Java generics, whose information is erased by the compiler after the type checking. SafeJava [7] could not support safe runtime downcasts to array types, since there is no way, while working on the language level, to provide an array object with an added owner field that can be accepted by the JVM at runtime. This is also the case with Cameron \& Noble [15] in treating array objects just like primitive types that are not required to be owned, while providing only the array elements with generic owners, whose presence does not violate the JVM.

In relation to the use of owners-as-modifiers, Universe type system (UTS) [25] handles array objects by providing two ownership modifiers, one for the array itself and the other for the array elements. UTS integrates ownership information to Java Modeling Language (JML) [34, 35], which facilitates runtime assertion checking or verification. Assertion checking ensures that an object meets an assertion during program execution. That 
is, an object can be assured to hold certain ownership information at runtime.

The fact is that Java arrays are objects and therefore need to be owned. Generic ownership is a mechanism for treating ownership information as supplementary generic types, but there is no generic array creation in Java. Java generics are a language-level mechanism, while Java arrays are checked at the JVM-level. Accordingly, OGJ $\mathrm{J}_{+}$forbids the use of traditional Java arrays. We created the final wrapper object OwnedArray, whose state is represented by a private one-dimensional array object. OwnedArray bundles operations that can: return an element at a specified position, replace an element at a specified position, return the number of elements, resize the capacity, and copy a segment from the current OwnedArray object to another destination object. OwnedArray can be used within an $O G J_{+}$program in the way illustrated by the example in Fig. 4.1

\subsubsection{The main() Method}

Since the commonly practiced Java array types are no longer available in $\mathrm{OGJ}_{+}$, the main () method is therefore adapted to capture the command line arguments in an OwnedArray object. The code in Fig. 4.1, as well as the code in Fig. 3.1, shows a runnable OGJ program. The static initializer in line 18 is mandatory as it invokes the operation begin (), which is responsible for translating the new main () method signature in line 19. As illustrated, the OwnedArray object used takes an OwnedString object as a type argument, and the actual owner parameters are all World, since the main method is a static context; this is enforced by OGJ as will be explained in subsection 4.3. Nevertheless, as the logic goes, the main () method is an entry point for running a program whose ownership hierarchy should be rooted at World. 


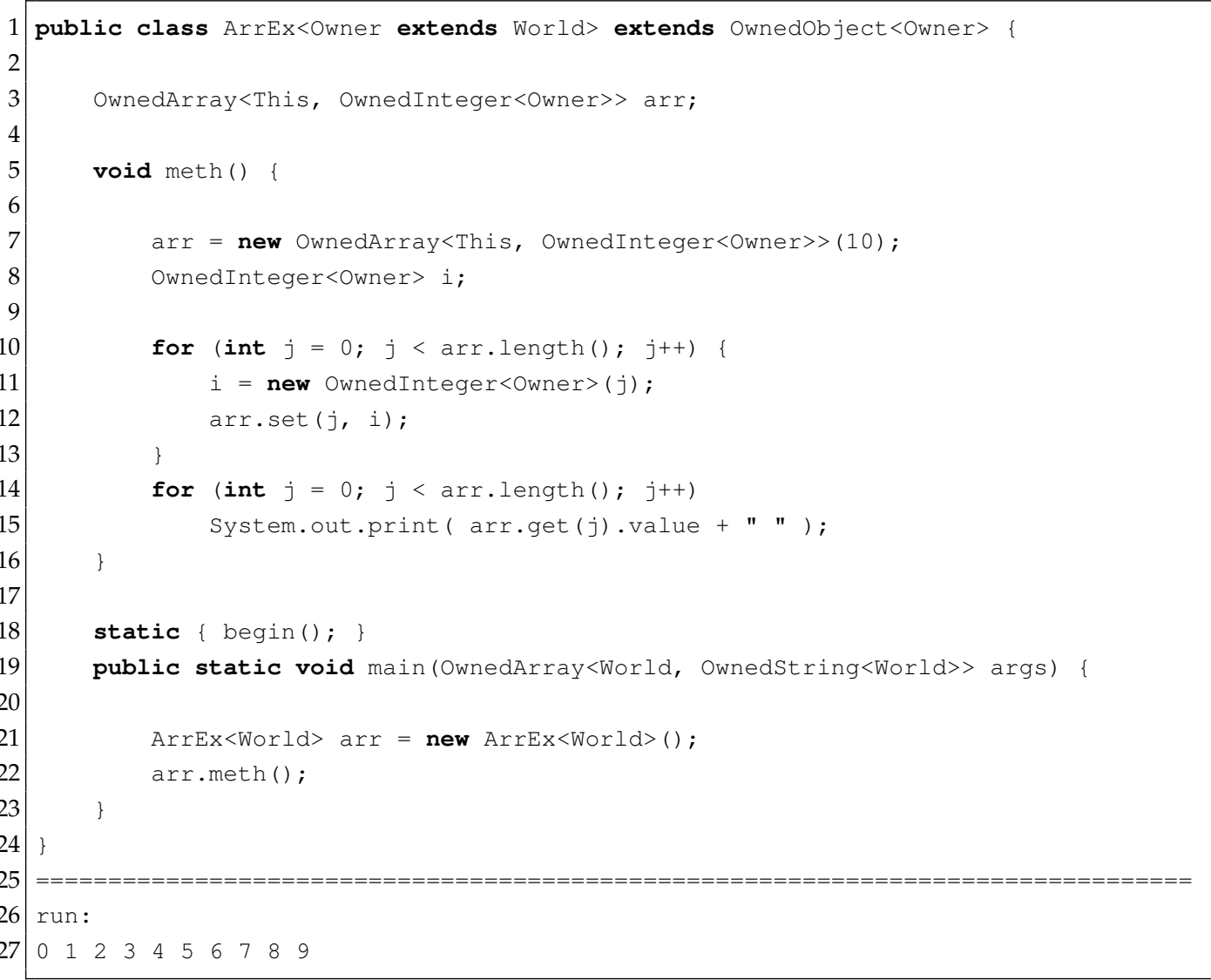

Figure 4.1: The Use of OwnedArray 


\subsection{Inner Classes}

Inner classes are non-static classes which are declared within other classes. Nevertheless, this nesting is only a relationship between classes, not objects. Java facilitates such nesting so that an inner class's instance can maintain an implicit pointer to the object of its enclosing class. This way, the state of the enclosing class is always available to its inner classes; although, as instances, each has its own independent identity.

As explained in subsection 2.3.1, the owners-as-dominators approach to encapsulation accommodates an equal treatment of inner classes and their enclosing classes, since inner classes are usually instantiated independently and therefore references from them to the outer class's representation are incoming references. That is, there should be no common representation between nested classes. For $O G J_{+}$to maintain deep $o b$ ject ownership, the interpretation of owner This is class-specific, since the meaning of owner This depends on the existence of receiver this. That is, an inner class cannot access fields declared in an enclosing class as owned by This. An inner class cannot also invoke methods defined in an enclosing class with formal parameter types and/or return types owned by This. See the erroneous statements in Fig. 4.2, where owner This in the outer class is not considered compatible with owner This in the inner class. Rationally, $O G J_{+}$allows a readonly wildcard owner parameterised reference to refer to objects in the outer class. 


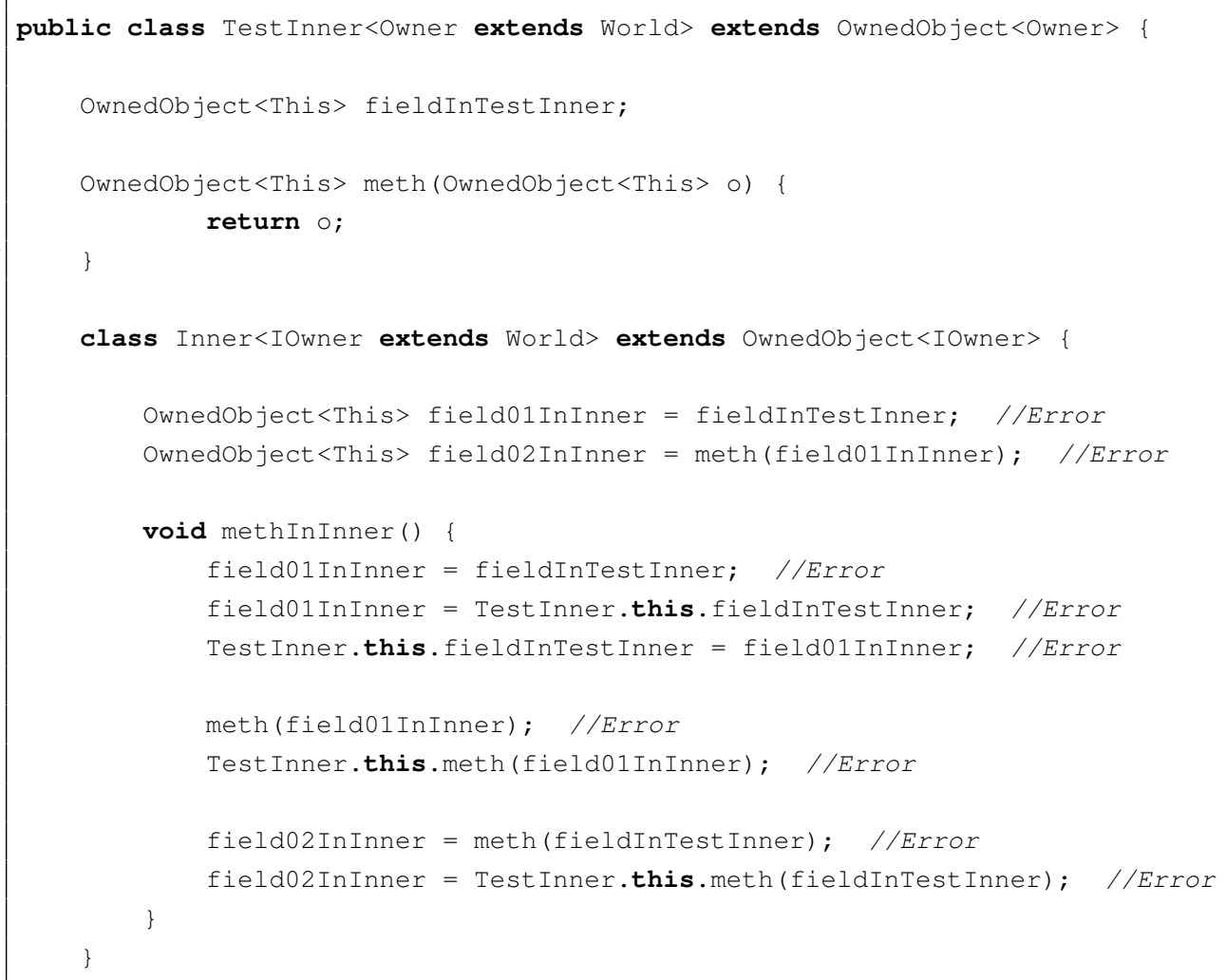

Figure 4.2: No common representation between inner classes and outer classes

Since the instances of inner classes are independent of their enclosing objects (i.e., the nesting is between classes, not objects), Java normally does not allow type variables declared in an inner class to be used in an outer class; however, Java allows vice versa. As a result, owner parameters declared in an inner class are safe from being used in an outer class, but not vice versa. For OGJ to maintain proper independency between object, the use of an enclosing class's distinguished owner in any of the enclosing class's inner classes is prohibited. This is because there should be no nesting relationship between the distinguished owner of an enclosing class and the distinguished owners of the inner classes. If we are to allow the 
use of the outer class's owner in an inner class, then the inner class will be able to define siblings to the outer class without the outer class's knowledge.

Although Java prohibits the explicit use of inner class's type variables in the enclosing class, generic methods facilitate a workaround, which $\mathrm{OGJ}_{+}$can catch. See the erroneous statement in Fig. 4.3. Owner must be inside MOwner; and by extension, Owner must be inside the owner that meth ( ) would infer. Since there is no nesting relationship between Owner and IOwner, the call to meth ( ) violates the requirements by Owner.

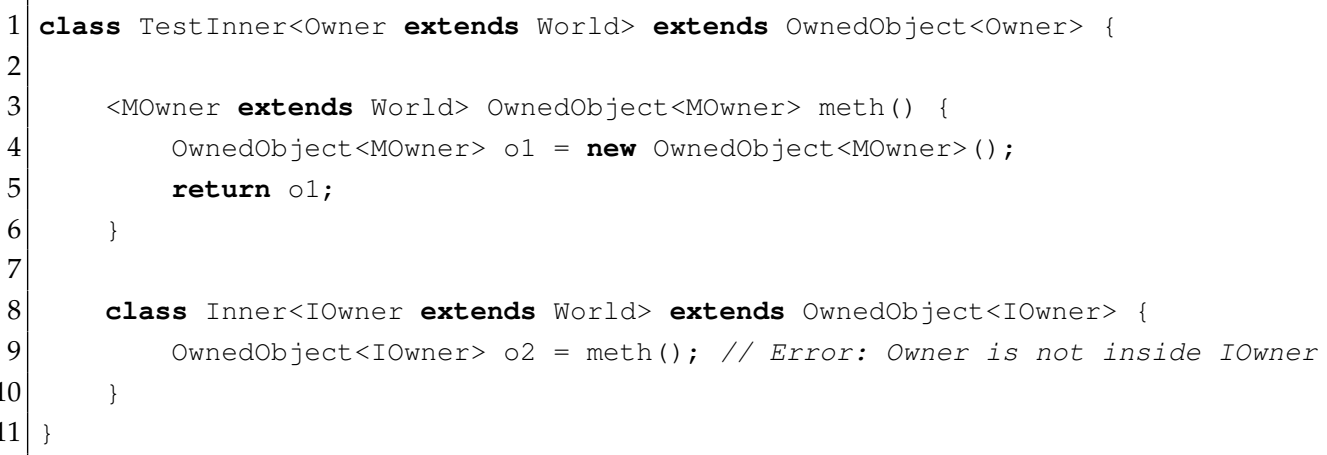

Figure 4.3: An outer class's owner is not inside the inner class's owner.

One limitation regarding the treatment of inner classes in $O G J_{+}$is that implementing event-listeners as anonymous inner classes is not possible in the way illustrated in Fig. 4.4, since $O \mathrm{OJJ}_{+}$will not allow the method invocation, in line 27, as long as the actual parameter is owned by This. However, event-listeners can be implemented safely as anonymous inner classes in $\mathrm{OGJ}_{+}$, in the way illustrated in Fig. 4.5, as long as the return type, in line 20, is owned by This. Consider the changes made in lines $2,10,11$ and 19 to provide the context covariance necessary to avoid restructuring the original code, if at all possible. As explained earlier in subsection 3.6.1 wildcard owners are mainly utilised in OGJ to facilitate context covariance, not only readonly references, and that is why a wildcard owner can only be bounded by World. 


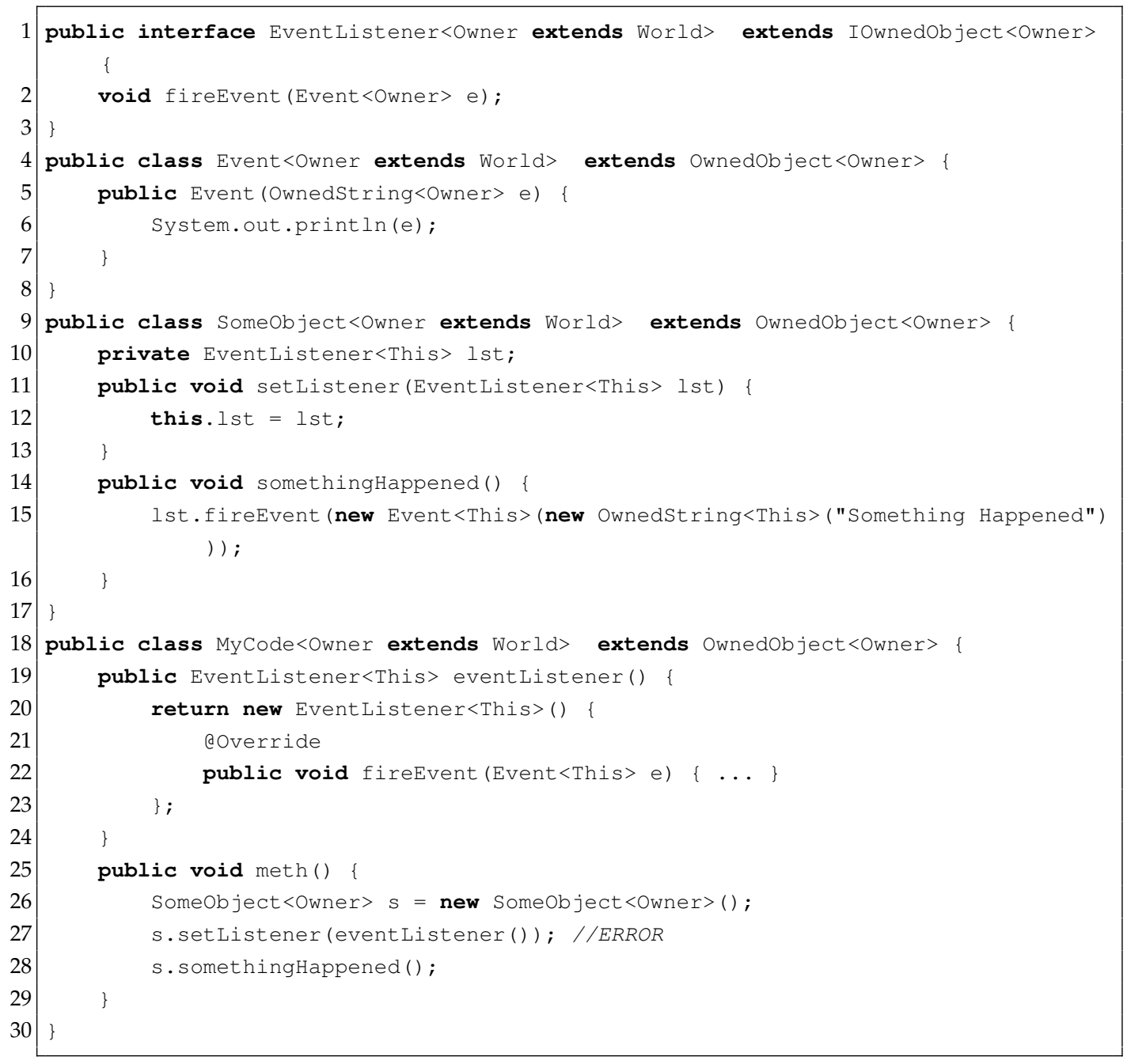

Figure 4.4: Event-Listener Pattern implemented in OGJ (uncompilable) 


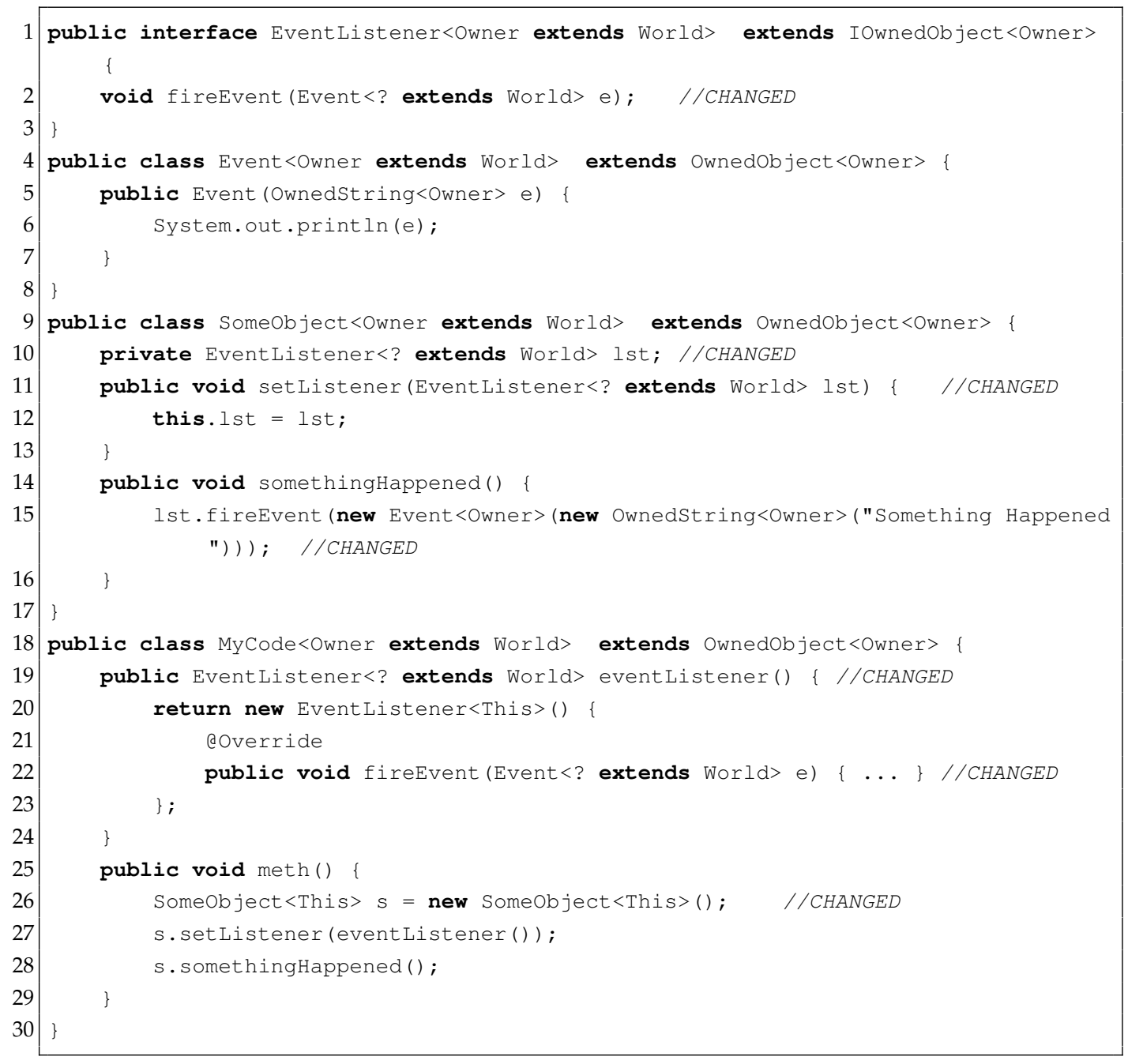

Figure 4.5: Event-Listener Pattern implemented in OGJ + (compilable) 


\subsection{Statics}

Java does not allow static declared member classes, methods and fields to use a type variable, and hence an owner variable, defined by the enclosing class. Moreover, if the enclosing class is a static member class, the same applies to its static members. This is because all type variables are nonstatic. Since a static member is shared along with its enclosing class, and since there should exist only a unique instance of that static member for all instances of the generic enclosing class, a static member needs to be accessible by objects located in any arbitrary ownership contexts.

The treatment of statics has not been specified explicitly in many proposals. Treating statics within the notion of owners-as-dominators [32] is not significantly different than within the notion of owners-as-modifiers [36, 44], as long as the object ownership heap graph is of only one tree structure. Huang and Milanova [32] force all static fields to belong to the root context, and assume that static methods have a virtual receiver this. Universe type system (UTS) [36, 44] does the same for static fields; but for static methods, the meaning of the peer modifier is slightly adapted. Normally, the peer modifier annotates an object as a sibling object; that is, a peer object has the same owner as the this object in order for both objects to share the same context. In the case of static methods, if a method is called as peer Class.method() at a static call site, then the callee's context will be the same as the caller's. If the same static method is called at a non-static call site, then the callee's context will be the same as the this object.

Summers et.al. [63] proposes extending the universe types heap structure with multiple trees, where each tree is rooted in a class. Classes can, therefore, own objects as static fields. This can be achievable since the root context of a UTS is the set of objects with no owner, and the root context is excluded from the verification of the nesting relationships between owners. In deep ownership, every single object should have an owner. 
If we are to have an individual tree for each object owning static fields, while forcing such an object to be owned by World, then we will end up with a single tree that does not preserve the nesting relationships. If we are to treat an object, that owns static fields, as non-owned or as virtually owned by World, then we will end up breaking the rule of having all objects owned, as well as breaking the nesting relationships. OGJ $J_{+}$ensures that a static field is either in the root context or wildcard-owned in order to guarantee correct object encapsulation, while sustaining the possibility of accessing static fields from any arbitrary context.

\subsubsection{Static Fields}

A static field is also known as a class variable; so, as the name implies, a class variable is a per-class incarnation rather than a per-instance incarnation. As a rule, Java does not allow the use of a type variable as an actual type parameter within a static field; accordingly, there is no way to have an owner variable as an actual owner parameter within a static field.

As for the use of owner This, since the significance of This relies on the presence of receiver this, owner This cannot be used within a static field declaration. Consider the example of Fig. 4.6, Java only provides a warning about "accessing static filed" in response to the assignment statement in line 7; and if meth ( ) is static, Java will provide an error because Java treats this as a non-static variable. The principle that should hold here is that each private static field-as a unique instance-must be accessible by any of the enclosing class's instances, no matter the location of these instances in the ownership tree. Although owner World can stand alone as a statically safe owner for static field declarations, we also have wildcard owners through which static fields can still be available for objects located in different contexts. A static field can then be declared private for proper information hiding, as need be. 


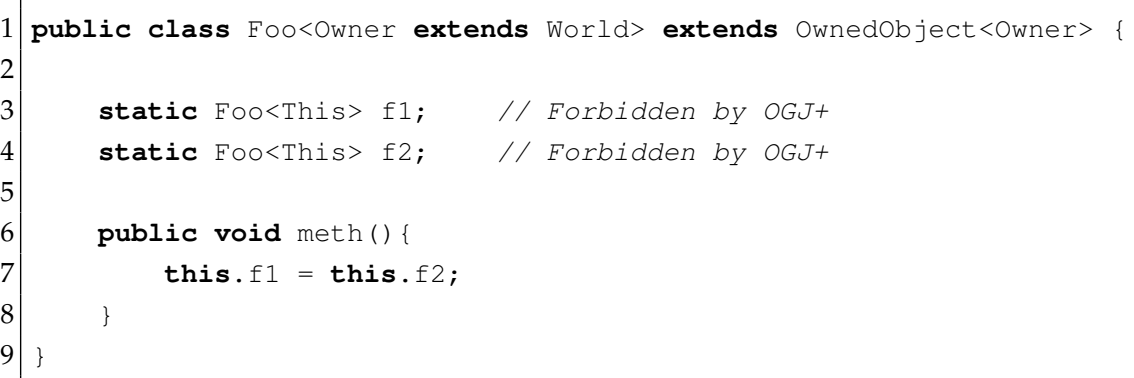

Figure 4.6: Illegal Static Field Declaration

\subsubsection{Static Methods}

Similar to static field declarations, owner variables declared by the enclosing class cannot be used within a static method, neither within the signature, nor within the body. Nevertheless, since a method can be owner parameterised, a static method's local variable or parameter can share contexts, as need be. An owner parameterised static method can be called, at a static call site, as Class. $<$ World $>$ method(), or as Class.<Owner>method (), so that the method can be evaluated either in the root context, or in the context of the caller, respectively. If the same static method is called at a non-static call site, then it can be called as above, or as Class. <This>method (), so that it can be evaluated in the context of the this object. If a static method is not owner parameterised, then it can only be evaluated within the root context, unless it involves wildcard owners. For the same reason as static fields, a static method cannot use owner This in the signature or in the body, as it should be available in arbitrary contexts.

\subsubsection{Static Blocks and Nested Static Classes}

Owner This is disallowed within static blocks and nested static classes. Methods declared inside these static contexts can be owner parameterised as need be. $O G J_{+}$treats anything declared within a nested static class 
as a static context. Inner classes, methods, blocks, and fields which are declared within a static class are not allowed to use owner This.

\subsection{Clone}

In languages that have reference semantics, the assignment operator is not appropriate for duplicating an object. Creating a duplicate copy of an object is sometimes essential for equality, or inequality, comparisons [29]. Copying an object is commonly conducted through a special default operation that is usually defined in the language's root class, as is the case with Java's java.lang.Object.clone(). The clone() method creates a new instance of the object that is being copied, then returns this new instance. All of the new instance's fields are aliases to the fields of the original object. Such an operation is conventionally known as shallow copy. The clone ( ) method's return type is ob ject; therefore, casting back the result to the original object is required. This default implementation can be overridden by some other custom behaviour in order, for example, to protect mutable fields from being affected by the behaviour of the original object. Normally, the override clone ( ) gets a copy of the original object by invoking super.clone () until it reaches object.clone(), which throws a CloneNot SupportedException if the class of the original object does not implement the interface $\mathrm{Cloneable.}$

Another alternative approach to object copy is deep copy, where the referents of all of the original object's fields are copied, not aliased. Deep copy causes no dependency between the source and target objects. So, the structure of the target object is isomorphic to the structure of the source object. On the contrary, shallow copy makes the target object completely dependent on the source, with no isomorphic structures. 
Grogono and Sakkinen [29] argue that copying operations should respect the semantic properties of objects rather than merely their syntactic properties, because "shallow" is too shallow and "deep" is too deep. In other words, the original and the cloned objects do not necessarily need to have isomorphic structures, and should not be completely dependent on one another. More specifically, objects which are not part of the private internal representation of the source object can be freely aliased within the cloned object; otherwise, references should be traced in order to copy their referents.

Noble et al. [51] discuss the idea of an ownership based mechanism called sheep cloning. Since an object owns its internal representation, the sheep cloning operation will copy all objects which are transitively owned by the source object, and will alias objects which are not part of the representation. It is not clear how the sheep cloning mechanism treats cycles within the object graph. There is an ongoing research aiming to formalize a type system that supports sheep cloning [38]. 
The example in Fig. 4.7 illustrates the implementation of the override clone () method used in the ArrayList collection class. Line 2 declares $\mathrm{V}$ as an object of type ArrayList whose initial value is a shallow copy of the current Arraylist. The Arraylist class is implemented as to have an array buffer to contain the elements of the ArrayList; which means that the backing array is constituting the internal representation of the ArrayList. Having the current ArrayList shallow copied, as in line 2, means that its backing array object becomes aliased, which is not safe. Therefore, the assignment statement in line 3 copies the backing array, elementData, into a new array object assigned to $v$. elementData. Since elementData forms the internal representation of the ArrayList, it should be owned by This in OGJ. . As a result, the field access on the left hand side of the assignment statement, line 3, would be erroneous in $\mathrm{OGJ}_{+}$, since elementData can only be accessed via receiver this.

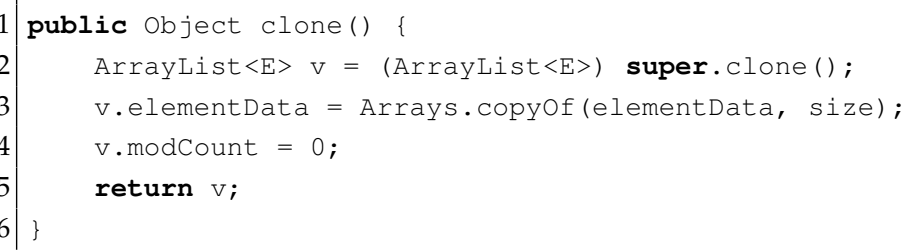

Figure 4.7: The ArrayList's override clone () method

The use of wildcard owners can mitigate the above mentioned restriction in the way illustrated in Fig. 4.8. A private getter method (line 1) can be defined to return a wildcard owner parameterised reference to the backing array. This reference can then be used inside clone () (lines 8 and 9) to copy the original array into the array of the clone. We used the method OwnedArray. copysegment () in line 9 instead of Arrays.copyof () (line 3, Fig. 4.7) which deals only with Java arrays. The receiver of copysegment ( ) is the source array; the arguments are, respectively: the starting position in the source array, the destination array, 
the starting position in the destination array, the number of array elements to be copied. Although implementing custom behaviours that deal with the This-owned objects is possible, shallow copying breaks deep ownership encapsulation, and hence should be forbidden in $\mathrm{OGJ}_{+}$because the clone will still be referring to the private representation of the original object; the statement in line 3, Fig. 4.7, and the statement in line 9, Fig. 4.8 . do not copy the actual elements but only references to the elements. Since immutable arrays exist neither in Java nor in $\mathrm{OGJ}_{+}$, facilitating deep copy is the appropriate solution for $\mathrm{OGJ}_{+}$.

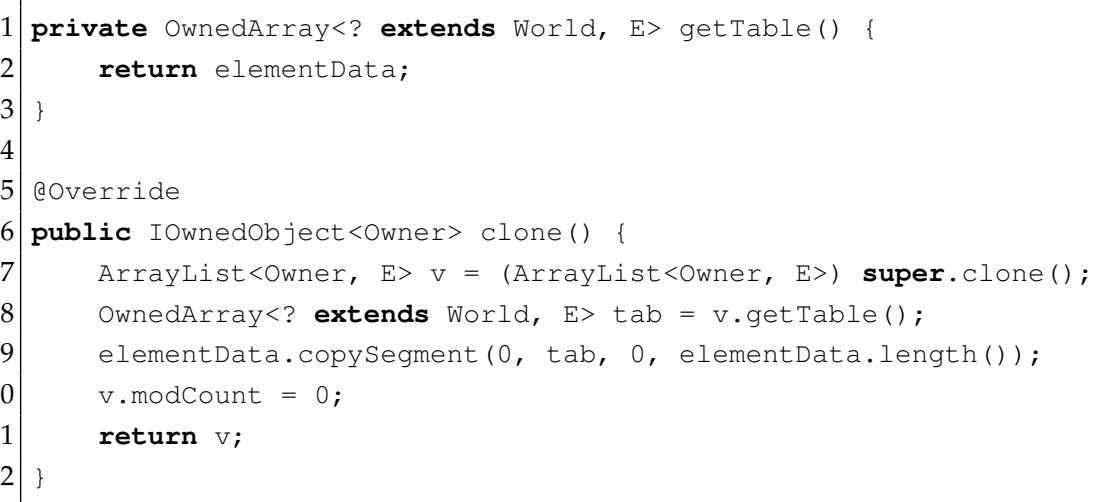

Figure 4.8: An unreal OGJ version of ArrayList's clone ()

We choose to facilitate an automatic version of deep copying. The deepCopy () operation in $\mathrm{OGJ}_{+}$can be overridden in a way similar to that of java. lang. Ob ject.clone (), so that custom behaviours can be implemented, as need be, to alias the objects outside the representation of the copied object. $O G J_{+}$requires the object being copied to be serialisable. This can be done by making all of the classes that make up the class of the source object implement the Serializable interface. Having the source object serialisable means that all of its values can be serialised and written into a ByteArray; then, the ByteArray can be deserialised into a new object, during which we can assign the required owner; that is, copies can 
be public, private or siblings; see lines 17, 20 and 23, Fig. 4.10. This approach takes care of the superclass fields, and traces the object graph to handle repeated references to the same object within the graph. There is no need to cast the result back to the source object, since deepCopy ( ) is a generic method, whose type variable's bound, IOwnedob ject, is wildcard owner parameterised. The example in Fig. 4.10 illustrates the use of the deep copy operations provided by $O G J_{+}$.

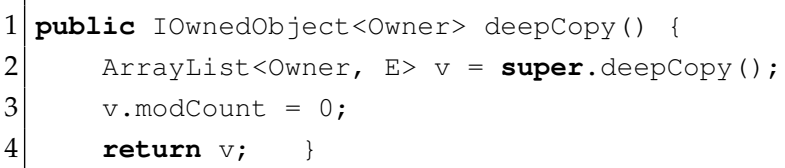

Figure 4.9: The OGJ+ArrayList's deep cloning operation

Consider Fig. 4.9 which is the cloning operation deepcopy () of the $\mathrm{OGJ}_{+}$ArrayList. In comparison with the clone () operation in Fig. 4.7. the backing array elementData does not need to be cloned again, because elementData was already deep copied by super. deepCopy () (line 2, Fig. 4.9) . Any other non This-owned object outside the private internal representation of ArrayList (e.g., modCount, line 3, Fig. 4.9) can still be accessed and aliased, as need be, in order to provide the required sheep (or sheep-like) cloning. The Arrays. copyof () operation in Fig. 4.7 shallow copies elementData; that is, creates a new array object but only references to the elements are copied, not the actual elements. Using OGJ+'s deepCopy ( ), everything is deep copied; and deep ownership encapsulation is guaranteed to be preserved, since the elements of an aggregate are part of its private internal representation.

The limitation of relying only on deepcopy () is that programming idioms which rely on shallow copying and on pointers to the internal representation of both the clone and the original object (e.g., to observe the changes since the clone was created) can no longer be implemented in $\mathrm{OGJ}_{+}$without being restructured to perform value comparisons rather than reference comparisons, if at all possible. 


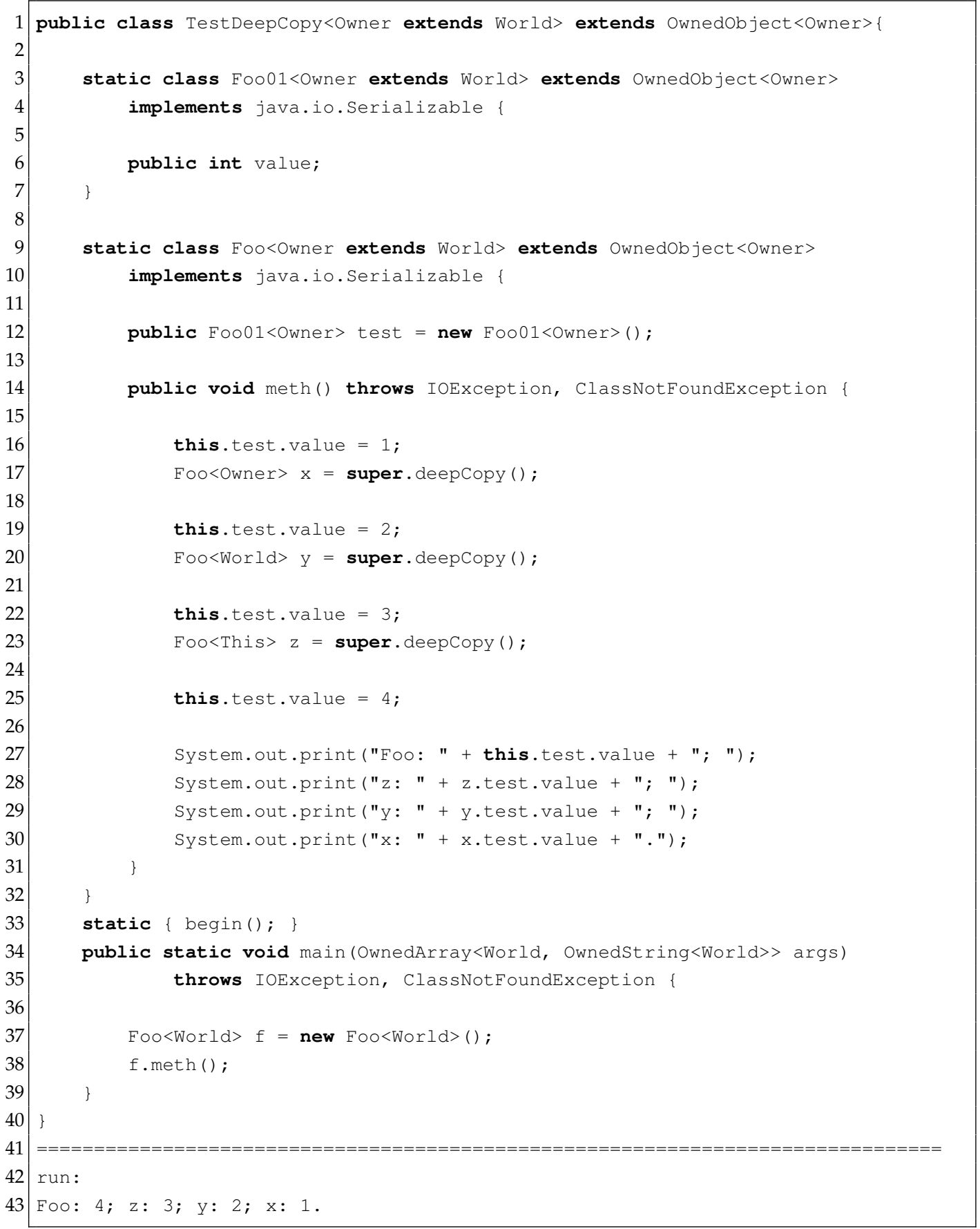

Figure 4.10: Deep copy operations in OGJ 


\subsection{Equals}

Java's equals ( ) method is another default operation defined in the root class Object. As the method's name implies, it performs equality comparisons. Nevertheless, equals ( ) operates in exactly the same way as the equality testing operator ${ }^{\prime}=={ }^{\prime}$, which performs identity equality comparisons rather than object equality comparisons. Similar to Java's clone ( ), the default implementation can be overridden by hand-coded versions. Java's equals ( ) method is mostly overridden by, recursively, comparing referenced objects with equals ( ) ; for instance, see Fig. 4.11, line 12.

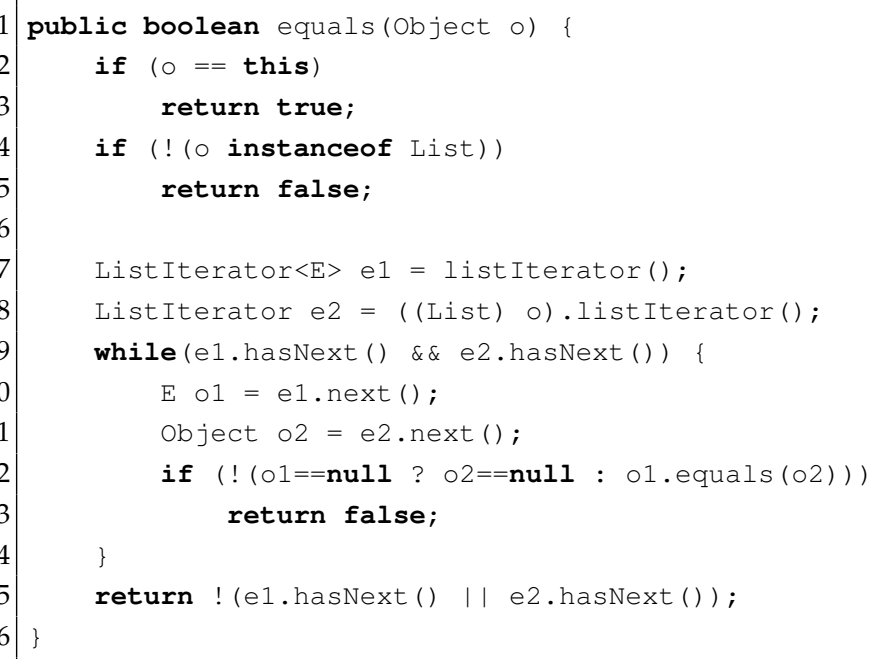

Figure 4.11: java.util. AbstractList. equals ()

The equals() signature is: boolean equals (Object obj). This means that the overriding implementation should check if the method's actual parameter is of the required type (line 4, Fig. 4.11); this is normally the type of the enclosing class, a superclass, or the abstract type it implements. If the check holds true, then it is inevitable to downcast the method's parameter to the verified type (line 8 ) in order to perform the required equality comparison between the receiver and the argument of the overriding implementation. This standard code reuse practice became 
entangled by the use of ownership types, since not only the object graph should be taken into account but also ownership information should be evaluated. Moreover, with deep ownership types, the nesting information should also be evaluated.

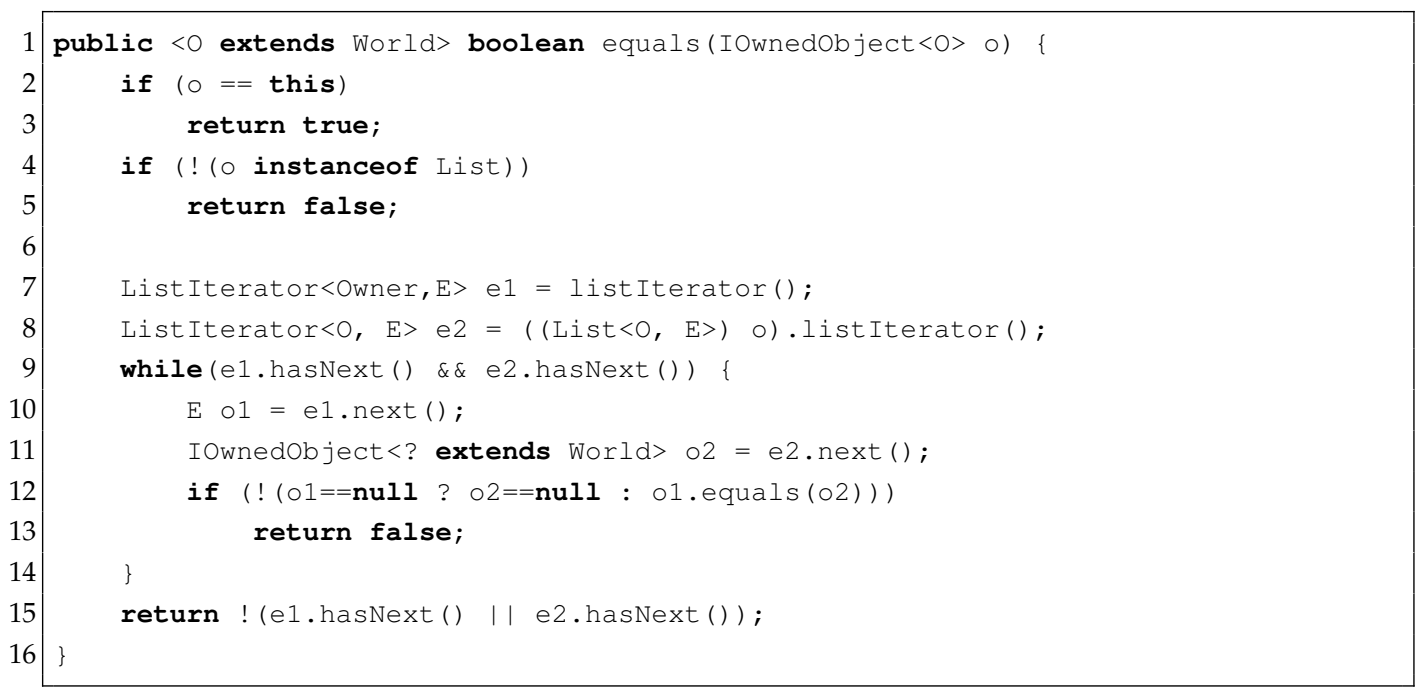

Figure 4.12: OGJ. java.util. AbstractList. equals ()

The example in Fig. 4.11 shows the overriding equals () implementation for the collection class java. util. AbstractList of Java 1.6. In line 8, consider the downcast of the method's parameter to the abstract type List. First, $\mathrm{OGJ}_{+}$does not allow raw cast since all objects should be owner parameterised. Second, passing a List object to the method means upcasting the argument to object, then downcasting it back to List, which is something that cannot be done in $O G J_{+}$as explained in section 3.5 by the example in Fig. 3.9. To overcome this restriction, $\mathrm{OGJ}_{+}$only permits this kind of downcasting if all of the additional outside owners are hidden via wildcards. The example in Fig. 4.12 shows the overriding implementation of equals() for OGJ.java.util.AbstractList. The latter class has the type variable $\mathrm{E}$ declared in its header with a placeholder owner as follows: $\mathrm{E}$ extends IOwnedObject<? extends World $>$. 
That is, the downcast in line 8 is allowed. In conclusion, the restrictive form of casting in $O G J_{+}$might seem to impose an impact on the way an equals () is written in $\mathrm{OGJ}_{+}$, but the simple decision of using a placeholder owner within the class header mitigated this impact. As illustrated by the example in Fig. 4.12, we did not need to restructure the example in Fig. 4.11, but did supply only owner parameters. Note also that the OGJ+'s equals () is owner polymorphic so that equality testing between objects with different ownership information can be conducted.

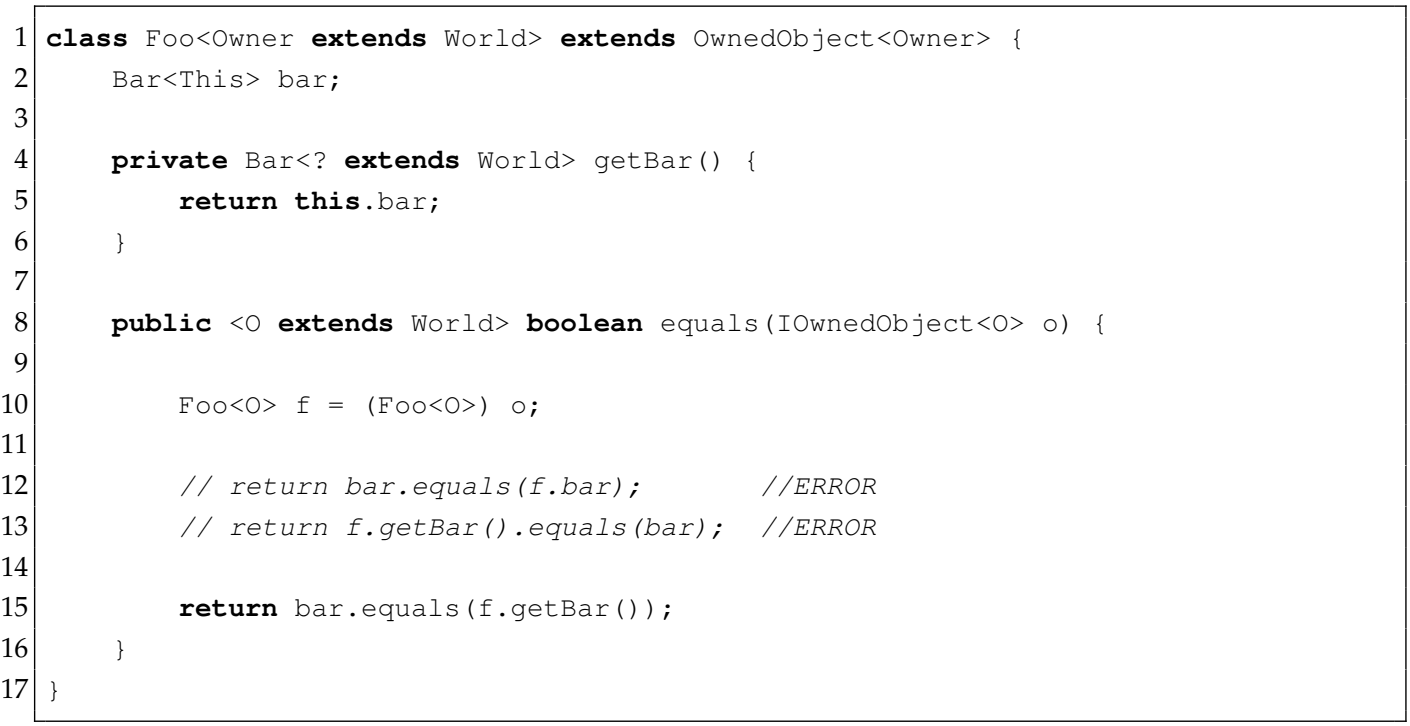

Figure 4.13: equals ( ) accessing two unrelated private objects

Consider the example in Fig. 4.13, the field access $f$. bar in line 12 is erroneous since bar is owned by This, and hence can only be accessed via receiver this. To overcome this restriction, we can define a getter method, such as getBar ( ) in line 4, and then invoke it as in line 15. Nevertheless, getBar ( ) cannot be used the way illustrated in line 13, since there is no guarantee that the returned wildcard-owned type is inside bar's owner, as explained in section 3.6. Moreover, $\mathrm{OGJ}_{+}$prohibits method invocations if the receiver and the actual parameter are both wildcard-owned. That, in fact, imposes a limitation on one's ability to use OGJ 's equals ( ) and 
method invocation in general (see Fig. 4.14, line 28); and would obstruct both the implementation and utilisation of some programming idioms and design patterns (e.g., caching pattern).

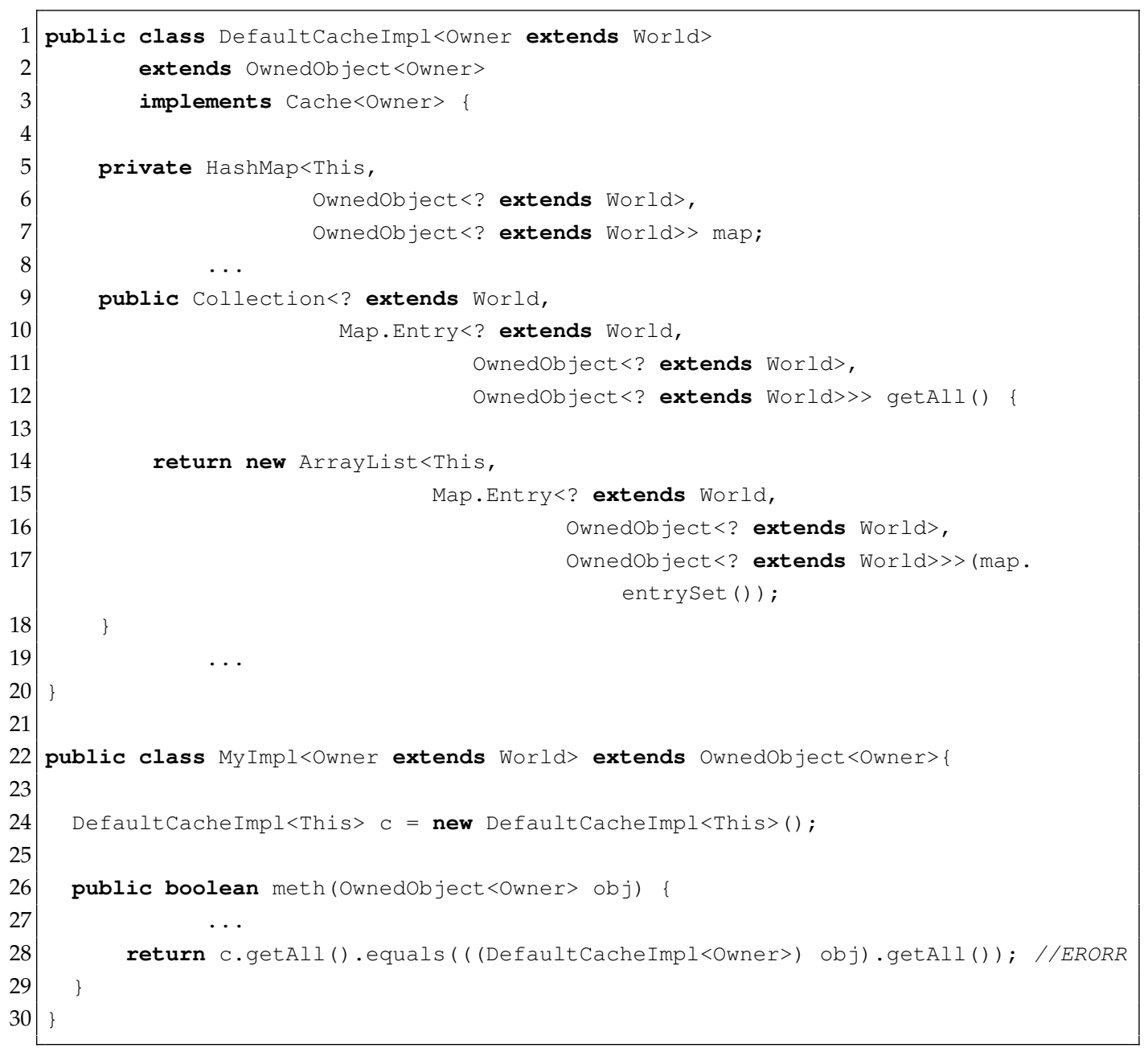

Figure 4.14: Illegal equals ( ) invocation (receiver and actual parameter are wildcard-owned) 


\subsection{Exception Handling}

Java utilises exceptions to handle errors and exceptional events that could happen during the operation of a method at runtime. Whenever such an event occurs, the relevant method creates an exception object and hands it to the runtime system; this procedure is called throwing an exception. Whenever an exception is thrown, the runtime system goes over the call stack (the chain of methods called to reach the method that has thrown the exception) in search of a method that contains an exception handler.

Exception handling in type systems that enforce ownership encapsulation properties raises the issue that an object may create an exception in one ownership context, while the object that has the exception handler might be in another context. Consider the example in Fig. 4.15, the object of class FoosFather owns Foo; and Foo owns FoosChild. FoosChild creates an exception object, at line 17, while Foos Father handles the exception. F०O is in the call stack, and is neither throwing nor handling an exception, as depicted in Fig. 4.16. Consider the object ownership structure in Fig. 4.17, FoosChild is in a different ownership context (Foo's context) than that of FoosFather. If method FoosChild.init () creates an exception object local to Foo's context, then the exception will not be able to propagate farther Foo's context boundary.

Dietl and Müller [24] explain and evaluate four approaches to exception handling in ownership type systems: 1) cloning exception objects from the context where they are created to the context where they are handled; 2$)$ transferring exception objects from the context where they are created to the context where they are handled; 3) treating exceptions as global data that can be accessed from all contexts; and 4) propagating exceptions via read-only references, which may cross context boundaries. The authors think that both global exceptions and read-only exceptions are more applicable alternatives, since both approaches do not lead to specification overhead, and do not increase the complexity of the type system signifi- 


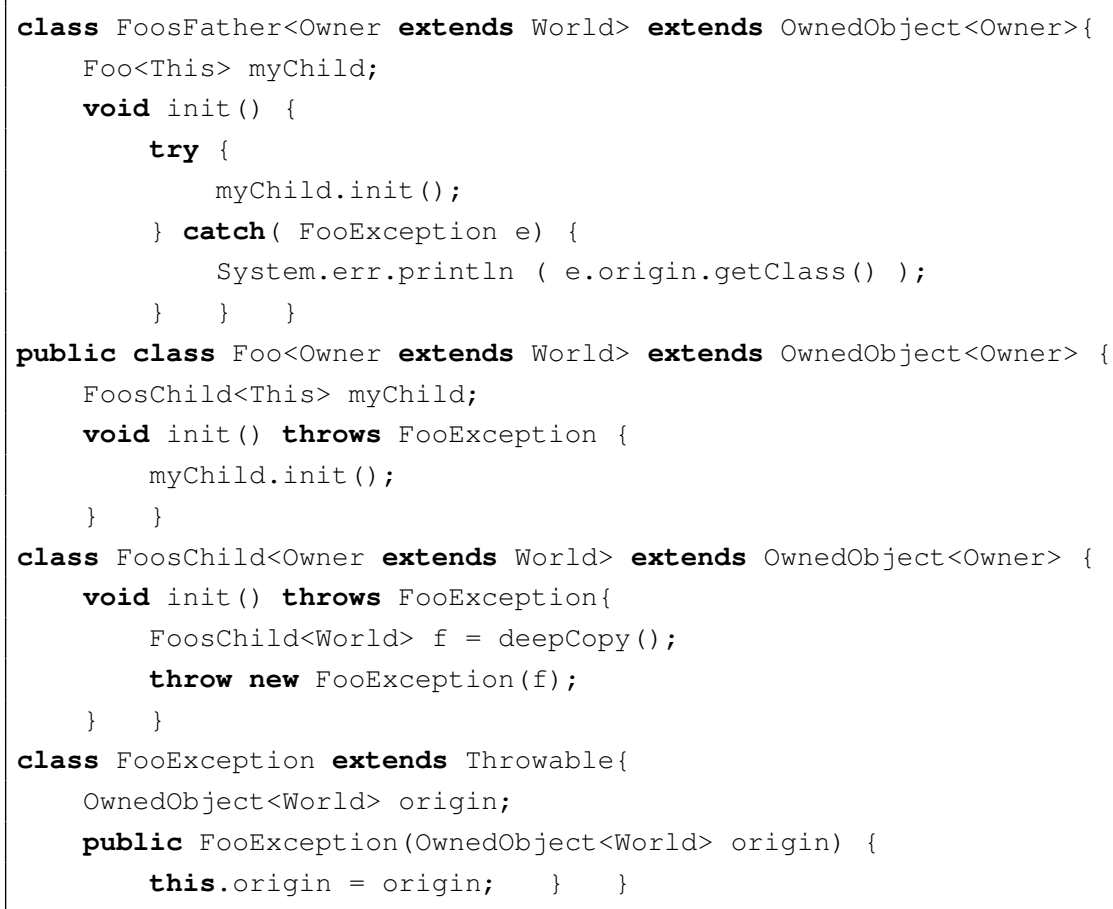

Figure 4.15: Exception Handling Example

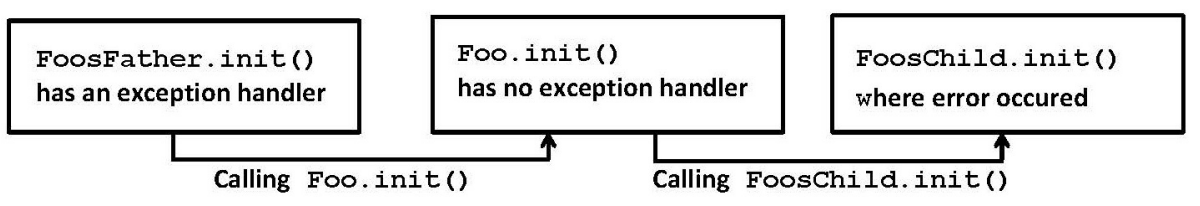

Figure 4.16: Depiction of the call stack exemplified in Fig. 4.15

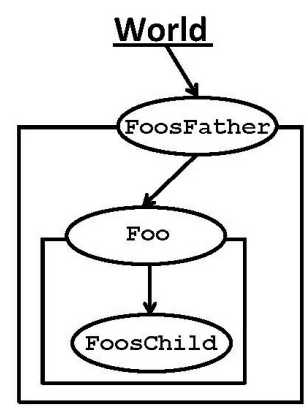

Figure 4.17: Depiction of the object ownership structure exemplified in Fig. 4.15 
cantly. The authors explain that read-only exceptions have two limitations. First, read-only references lead to a weaker ownership invariant. The second limitation is that an exception object is modified while being propagated. Handling this would require cloning the exception, modify the clone, then throw the clone. In relation to global exceptions, the declaration and propagation of exceptions are straightforward. Global exceptions are viable for ownership type systems that permit references to objects in ancestor contexts, as is the case with deep ownership.

$\mathrm{OGJ}_{+}$treats exception classes the same way as static declarations, where owner World and wildcards are the only allowed owner parameters within an exception class. If the example in Fig. 4.15 is implemented with wildcard owners used in lines 20 and 21, then we could have normally replaced $f$, in line 17 , by this and removed line 16; this is $O G J_{+}$'s best option. The example uses owner World within FooException; that is, we cannot use this in place of $f$ in line 17 , since casting this to OwnedOb ject $<$ World $>$ is not possible; owners do not match. Using the default deepCopy ( ) operation provided by $\mathrm{OGJ}_{+}$, as in line 16, allows us to get the required public deep copy of the current this. 


\subsection{Enum Types}

An enumeration type is mostly declared to contain a set of named constant values. Nevertheless, in Java, it is more appropriate to say that an enumeration type contains a set of closely related elements; for example, Colour $\{R E D, B L U E$, YELLOW $\}$. This is because an enumeration type in Java is a distinct compiler-generated class, not an arithmetic type, whose constant values cannot be directly set to numeric values.

Java enum types, or enums, were introduced in the type safe JDK 1.5, as a type safe facility, along with genericity. Nevertheless, enums cannot be generic types, although enums preserve type safety by implicitly extending the abstract generic class java.lang. Enum, whose header is as follows:

public abstract class Enum $<E$ extends Enum $<E>>$ implements Comparable $\langle E\rangle$, Serializable

The class Enum is self-referencing in its generics, and implements Comparable whose type argument is Enum's type variable. This ensures that an enum of one type can only be compared with another enum of the same type.

Since enums extend java.lang.Enum, enums cannot extend any other class or enum, as there is no multiple inheritance in Java. Enums are implicitly final; thus cannot be subclassed. Also, an enum is implicitly static when being a member of a class. Enum constants are implicitly static final. There is no way to assign something other than the predefined constants to an enum variable. Since enums are classes, it is possible to define constructor, methods, variables, and member classes inside Java enum. Nevertheless, a constructor must be declared private, to preserve the property that an enum is neither allowed to be instantiated nor extended. 
Since an enum class, as well as its constants, cannot be compromised via the getter/setter idiom, the safety measures that $O G J_{+}$applies are directed to any variable, method, or member class declared inside an enum class. What applies to anything declared outside an enum is applied to everything declared inside an enum. If an enum is declared as a member of a class, which is mostly the case, then OGJ + will treat the enum, and anything declared inside it, as being in a static context. An enum cannot extend classes but can implement interfaces; and therefore, an enum can only implement IOwnedOb ject, or its descendants. 


\subsection{Implementation Methodology}

This section describes the implementation approach adopted to extend Java with the encapsulation system described above. The implementation is done on the javac open source compiler.

\subsubsection{Ownership Domains and Types}

The ownership domains OGJ. Owners. World and OGJ. Owners. This are implemented as blank interfaces. This inherits World, which inherits Object. The root class OGJ. OwnedObject is an owner parameterised class, which implements the owner parameterised root interface OGJ.IOwnedObject. The default equality test operation equals() is declared in IOwnedObject, and thus is overridden in OwnedObject. Ownedobject also contains the default copy operation deepcopy (), which is declared protected.

The default array type in OGJ, OGJ . OwnedArray, is declared $\mathrm{final}$, with a public constructor that takes an integer parameter as the initial array size, then initialises a new array object through a private constructor. OwnedArray takes an owned type argument, as the type of the elements, in the way explained in section 4.1, then creates a normal Java array type and stores its value as a private field, which can be initialized by the private constructor and accessed directly. OwnedArray is declared serializable in order to conform with deepCopy ( ) that uses Java serialization, as explained in section 4.4. OwnedArray bundles the following operations: get (), set (), length (), resize (), and copysegment ().

Classes in the Java standard library (e.g., Integer, Number, String, etc.) have their corresponding classes in $\mathrm{OGJ}_{+}$(e.g., OwnedInteger, OwnedNumber, OwnedString, etc.); they are all owner parameterised, serializable, and have public constructors. 


\subsubsection{Type Checking}

The $\mathrm{OGJ}_{+}$code is type-checked through an extension to the Java Compiler (javac). javac is a program to put Java language source code files (. java) into an intermediate representation (IR), which is an abstract language independent of the specifics of a particular machine (platformneutral). The IR of a Java program is known as bytecode. The compiler processes source files to generate output machine-readable executable bytecode class files (classname.class). For each class defined in the source code file(s), the compiler creates a class file. So, Java class files, or bytecode classes, are the accepted form that can be converted, by the Java Virtual Machine, into native platform-specific executables. In that sense, javac is typically a front end of a traditional compiler, which parses source code in order to generate IRs.

For the compilation process to get started, the JavaCompiler class, which is defined in com.sun.tools. javac.main, will be invoked to read the source files specified on the command line. The compiler, first, enters a parsing phase, in which it processes source files with the help of classes defined in com.sun.tools.javac.parser.*. The parser will then call the lexer in order to generate a stream of tokens from an input stream of characters, then will map them into an abstract syntax tree (AST); this can be thought of as an advance towards the utilization of the package com.sun. source.tree.

As far as $\mathrm{OGJ}_{+}$is concerned, javac employs the AST to symbolize and process programs at compile time; and eventually, the AST is transformed to Java bytecode and written to a class file(s). The root class for AST nodes is com. sun.tools. javac. tree. JCTree, which implements com.sun. source.tree. Tree. To work with a tree, it is essential to traverse it somehow; javac utilizes the design pattern Visitor. The visitor class for trees is com. sun. tools. javac.tree. JCTree.Visitor, which encloses a number of visitor methods to carry out attribution on the AST. OGJ ${ }_{+}$injects only a couple of visiting messages, before and after 
attribution, into the JavaCompiler class. The visitor methods which are overridden by $\mathrm{OGJ}+$ are as follows:

- visitVarDef (JCVariableDecl tree)

Deals with variable declarations (fields, variabls, parameters).

- visitMethodDef (JCMethodDecl tree)

Deals with method declarations.

- visitClassDef (JCClassDecl tree)

Deals with class declarations.

- visitApply (JCMethodInvocation tree)

Deals with method invocations.

- visitSelect (JCFieldAccess tree)

Deals with field accesses and assignments.

- visitTypeCast (JCTypeCast tree)

Deals with type casts.

- visitTypeApply (JCTypeApply tree)

Deals with parameterised types.

- visitTypeParameter (JCTypeParameter tree)

Deals with formal class parameters.

- visitAssign (JCAssign tree)

Deals with assignment statements.

- visitNewClass (JCNewClass tree)

Deals with new (...) operations.

- visitReturn (JCReturn tree)

Deals with return statements.

- visitBlock (JCBlock tree)

Deals with statement blocks.

Each of the above methods is overridden to behave, and perform checks, as explained in a relevant section or subsection within the thesis. For example, in relation to nesting verification, subsection 3.4.2 describes 
part of the behaviour of visitvarDef (); in relation to instantiation and casting, section 3.5 describes the dehaviour of visitNewClass () and visitTypecast (), and so on.

\subsubsection{Testing}

OGJ implementation is, by all means, a Test-Driven Development (TDD). The process is mainly depending on reiterating brief development cycles. At the beginning, a test case, that contains a faulty $O G J_{+}$construct, is written to define the required new function, or modification; then, the code is written to satisfy that test. This process requires automated unit tests to verify that all added functionalities are still working after code changes. We used the JUnit testing framework as a test case execution tool for $\mathrm{OGJ}_{+}$.

\section{Test Cases}

Most of the test cases are moderately small; they range in length from 3 to 100 lines of code. The length varies mainly depending on the integrity of the number of errors that javac error log can report for each $O G J_{+}$ example program (test case). Sometimes, one test case cannot comprise all of the potential deviations for the same construct; it is therefore at times essential to have more than one test case for the same construct. We have more than 70 Java class files to test error reporting; some of these files can report up to 50 errors; some others contain correct $O G J_{+}$code to detect problems with the type checker. That is, there are two kinds of tests: failing tests and passing tests. A failing test checks that a given file does indeed fail, as should be; a passing test verifies that a given file does compile, as should be. Furthermore, applying OGJ $J_{+}$to the Collections Framework has turned out very useful in detecting subtle bugs that would otherwise have gone unnoticed. 


\subsubsection{Usage}

A minimal runnable $O G J_{+}$program should have the lines shown in Fig. 4.18. The Compiler is available for the Windows platform from the following link:

http://homepages.ecs.vuw.ac.nz/ ahmkhal/

To run $\mathrm{OGJ}_{+}$, Java should be run from the command prompt as follows:

$\mathrm{x}: \backslash>j a v a$-jar "OGJ_JDK6_v20130630.jar" "Class.java"

See subsection 4.1.1 for discussion on OGJ 's main () method (and thus the begin ()).

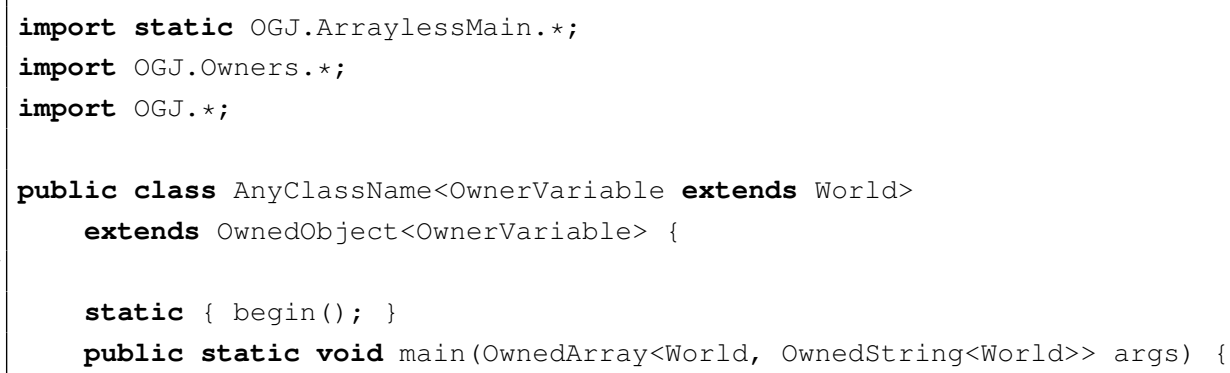

Figure 4.18: Mandatory declarations required for a minimal runnable $\mathrm{OGJ}+$ program 


\section{Chapter 5}

\section{Generic Ownership Compliant Collections}

To evaluate the applicability of $O G J_{+}$'s encapsulation system to a real life code base, we decided to refactor the JDK 1.6 Collections Framework. We found that Java Collections Framework is large, complex and important enough to be representative of the encapsulation issues we are targeting (e.g., the iterator pattern). Not to mention the fact that ownership was first introduced to target aggregation and container objects; and in the end, the refactored framework is in fact integrated to the new language. That is, $\mathrm{OGJ}_{+}$has its own collection library.

The Java Collections Framework [1, 5, 49] was introduced in JDK 1.1, and then redesigned more thoroughly in JDK 1.2. With generics introduced in JDK 1.5, a collection object became restricted to hold a particular data type, since the only thing a collection knows it holds was a handle to an object. Finding out type mismatches at compile time is known as compile-time type safety, which relieves programmers of the burden of casting when reading elements from collections. So, what we are about is supporting owner detection, as companion to type detection, through generic declarations.

In terms of encapsulation, a collection object can have its state compro- 
mised via an incoming reference to its internal representation. How? One of the very short, but most substantial, answers is: Iterators. The idea is that for a collection object to be able to share its element objects, an iterator needs to access the elements stored in that collection. As explained in subsection 2.3.1, an iterator can bypass the collection's interface and refer directly to any arbitrary element by maintaining an incoming reference to the collection's private representation that can in turn be mishandled via public getter/setter methods.

With ownership types, it is possible to have the internal representation explicitly declared as owned by its respective collection class. That is, a collection's representation is hidden: constrained to interact only with its owner collection object, with no incoming aliases.

This chapter explains how we refactored the JDK 1.6 collections' code base, in order to use OGJ,'s ownership support. The classes under study are the ones which are circled in Fig. 5.1 and Fig. 5.2. These classes include all of the general-purpose implementations, namely Arraylist, LinkedList, ArrayDeque, Priorityqueue, HashMap, LinkedHashMap, TreeMap, HashSet, LinkedHashSet, and Treeset. Also, the legacy implementations Vector and Hashtable will be investigated, as well as the special purpose implementation Ident ityHa shMap. The non-circled components in Fig. 5.1 and Fig. 5.2 are (1) the abstract data types, or interfaces, of which the collection classes provide implementations, and they appear in red colour; and (2) the extended abstract classes that provide the skeleton implementations of the relevant abstract data types. All of these classes and interfaces are subject to refactoring.

Typically, the refactoring of a class (or interface) starts off by declaring a distinguished owner for the class itself, then the appropriate owner parameters and bounds are supplied to the type parameters, as explained earlier in the thesis, and as will be explained later in this chapter. If the class is not extending another class in the hierarchy depicted in Fig. 5.1 (or Fig. 5.2), then the class should extend OwnedOb ject and the owner 


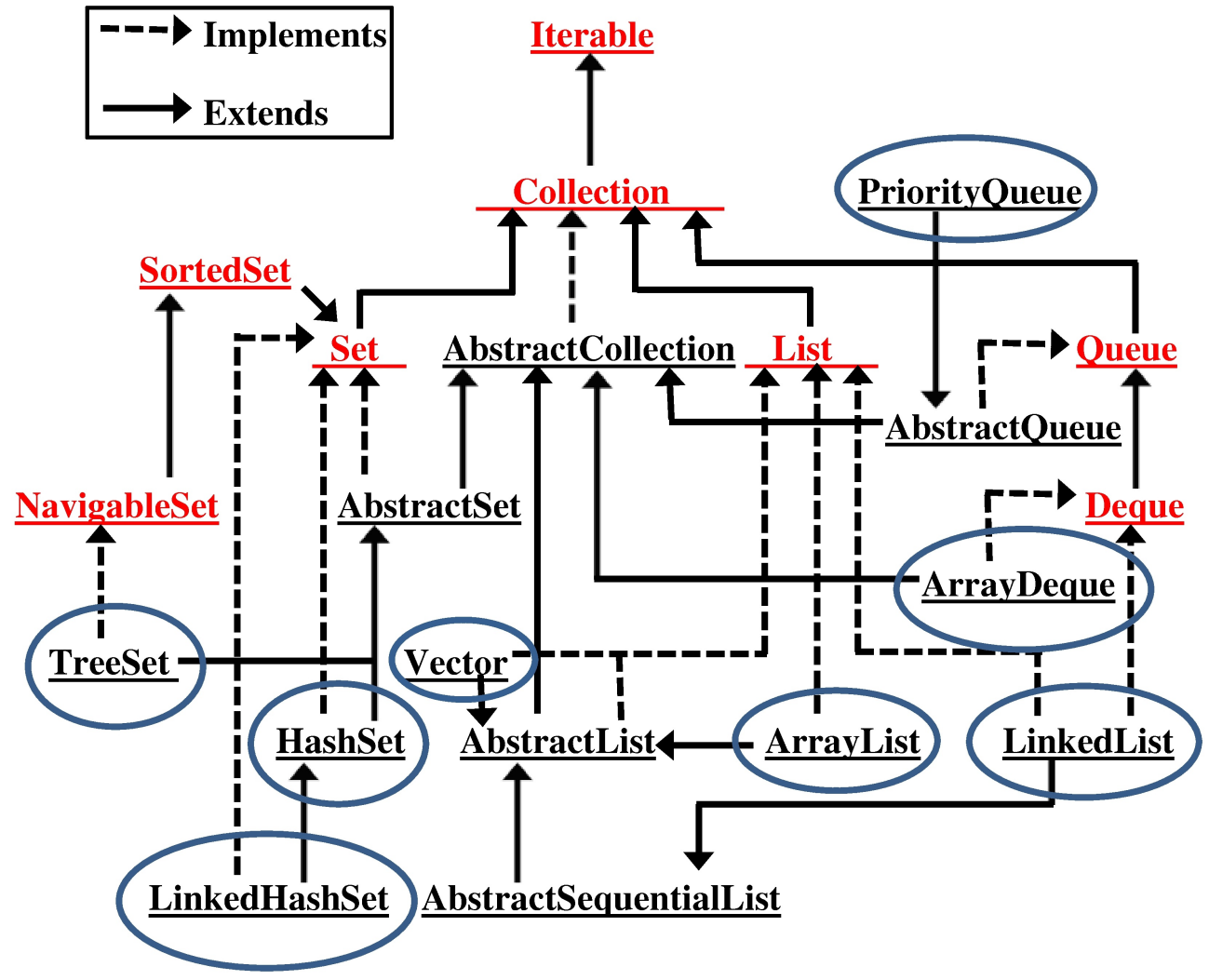

Figure 5.1: Iterable Collections

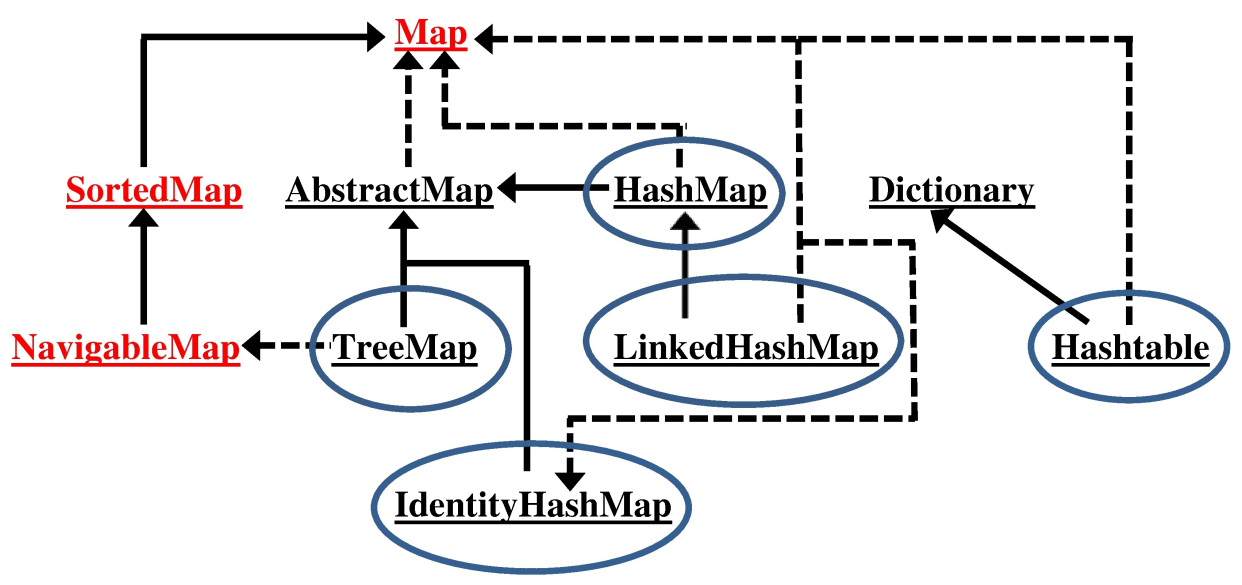

Figure 5.2: Maps 
of the class should be preserved as explained earlier in chapter 3. In a similar manner, an interface should extend IOwnedOb ject, if it is not extending another interface in its respective hierarchy. Refactoring the body of a class starts off by identifying its representation part and making it owned by This (i.e., to be private and hidden). Owner This will not allow any access to that representation part unless through variable this. The other non-representation objects will be initially siblings owned by the same owner as the defining collection class, which means that these objects can only be shared with objects owned by the same owner as the current instance of the defining collection class. Exceptions occur when, for example, a representation object is originally being assigned to any of the other objects. In this case, such objects will need to be made owned by This, and thus they will need to be only accessed via variable this, which at times is not the originally intended receiver. Here we come to what we call cross incompatibility between owners. The remainder of this chapter explains the modifications found necessary, and why a particular object had to become private, sibling, public or subject to context covariance.

Identifying the representation part for this particular refactoring was straightforward, as we considered every private field, in the original implementations, a representation object. Otherwise, the determination of the representation part of an object relies on human judgement, if not design principles.

Since most collection classes have compile-time dependencies on other classes in the framework, the priority was always to make sure that the whole set of classes under refactoring can altogether be successfully compiled with $\mathrm{OGJ}_{+}$, after every modification cycle. For rapid error reporting for that whole set of classes, we utilised the JUnit testing framework which provides an automated infrastructure for running repeatable tests. That is, the collection classes were dealt with as passing test cases that should altogether produce no errors. Modifications to one class can cause another 
to fail to compile, while the former can still pass the compilation. Thus, the integrity, and quality of having unconflicted set of class relationships, was a priority during every implementation cycle.

\subsection{Interfaces}

The set of classes mentioned above are descended from the two main collection interfaces: Collection (Figure 5.1) and Map (Figure 5.2). The set of interfaces and classes involved in both hierarchies are in the package java.util. This study is mainly concerned with five abstract data types: List, Queue, Deque, Map, and Set. These general purpose collections can be classified into iterable collections and maps. Iterable collections are those interfaces that extend collection; and by extension, their implementor classes. Maps are all interfaces and classes which are rooted by the interface Map.

Although the interface Iterable (in package java.lang) is outside the Framework, Collection extends Iterable so that lists, queues, and sets can be used with the foreach statement. It is not possible to iterate over a Map object directly; instead, Map provides Collection views so that a Map object can be iterated over through these views. That is, maps are not iterable collections; and thus, there is no need for Map to extend Iterable. 


\section{CHAPTER 5. GENERIC OWNERSHIP COMPLIANT COLLECTIONS}

\subsubsection{The Collection Interface}

To apply Generic Ownership to the interface Collection, the appropriate owner parameters and bounds are supplied to the interface header as shown in line 1, Fig. 5.4. Fig. 5.3 and Fig. 5.4 are the original JDK 1.6 and the $\mathrm{OGJ}_{+}$compliant versions of the interface collection, respectively.

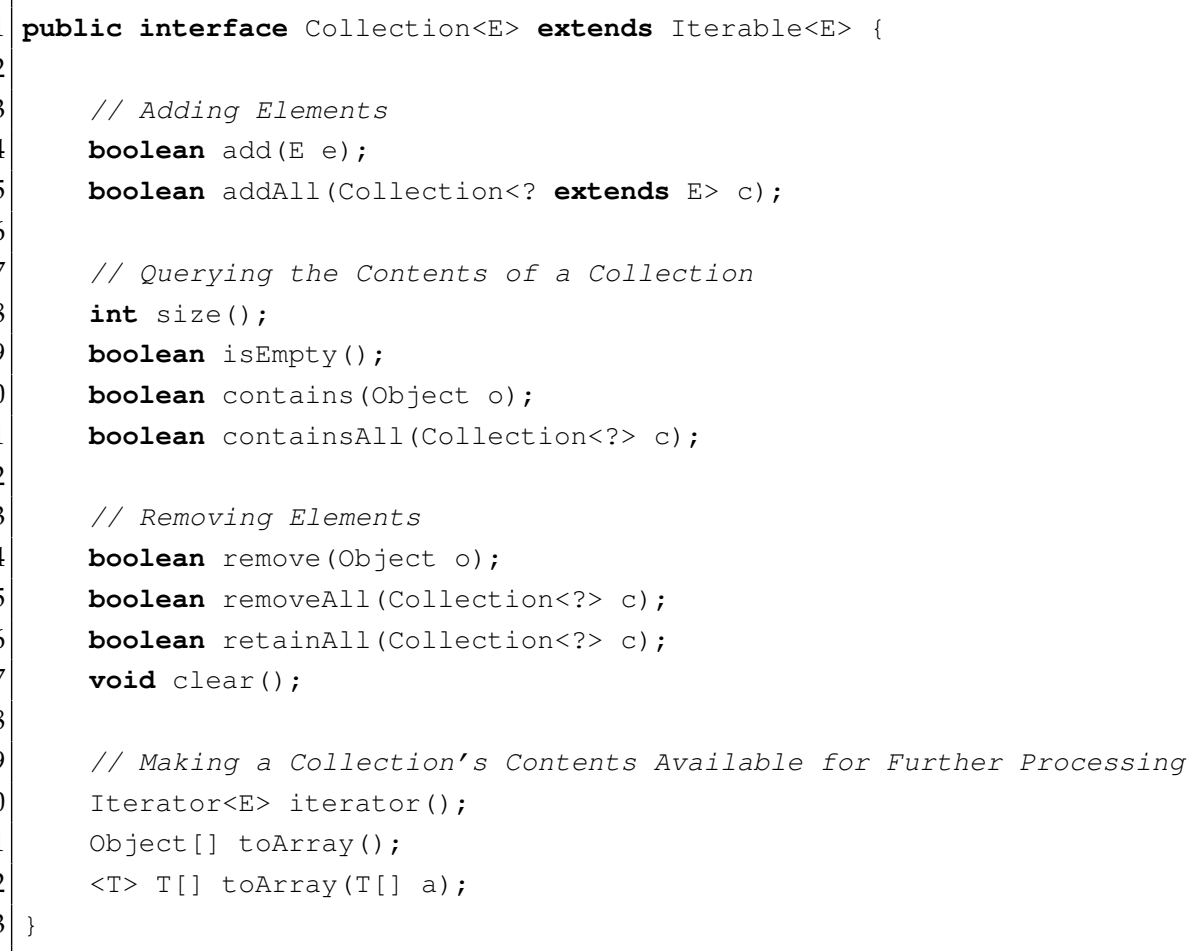

Figure 5.3: JDK 1.6 Collection Interface. 


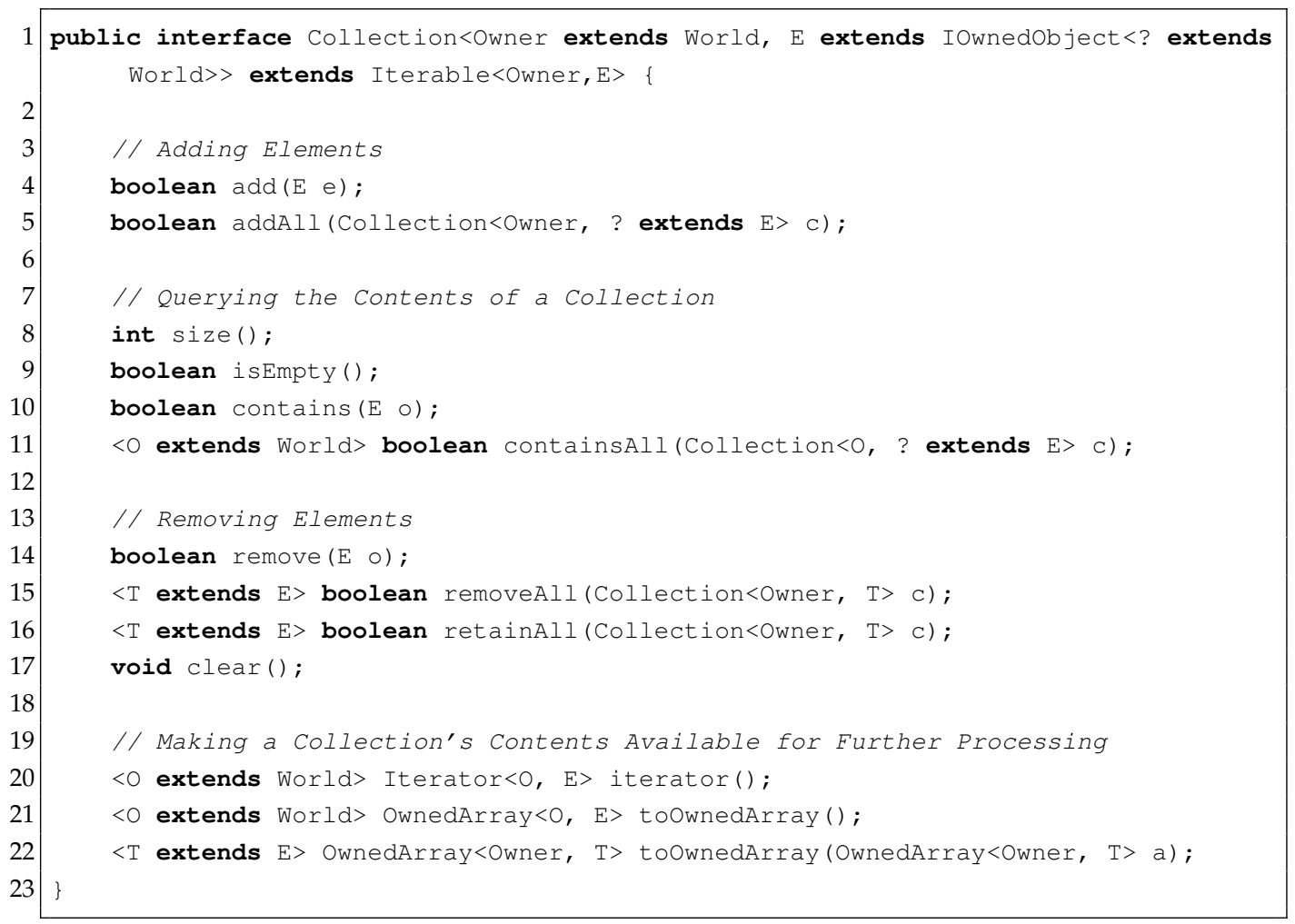

Figure 5.4: OGJ+ Collection Interface.

The OGJ + Collection header has Owner as the owner of Collection, and $E$ as the type of the elements. $E$ is bounded by IOwnedObject which is the root interface in $\mathrm{OGJ}_{+}$, and which Ownedobject implements, so that classes and interfaces can be used as bound objects. The owner of the elements is subject to context covariance (recall subsection 3.6.1). Collection extendes the interface Iterable, which must be within the same ownership context as Collection, and thus is owned by Owner. As for the definition of Iterable, it extends IOwnedobject and it reads as follows:

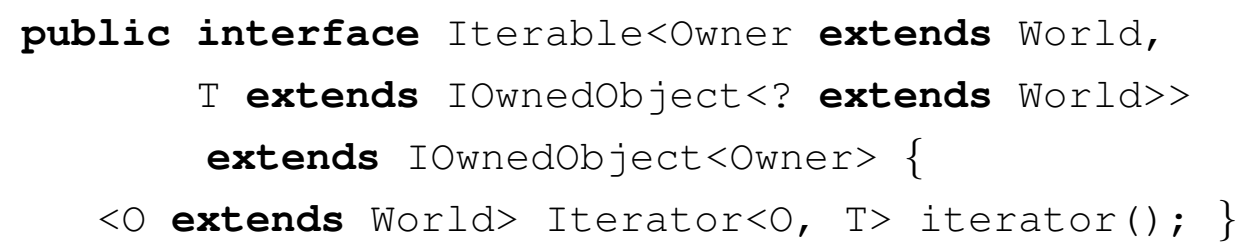


According to Naftalin and Wadler [49], the methods which are defined by Collection can be classified into four groups in accordance with their functionalities: adding elements, querying the contents of a collection, removing elements, and making a collection's contents available for further processing. In the rest of this subsection, I will clarify the required adjustments in the signatures of these methods.

\section{Adding Elements}

These are the methods in lines 4 and 5, Fig. 5.4. The add () method did not require any modification in the signature. For $\operatorname{addAll}()$, other than supplying the parameter type with the relevant owner parameter, there are no significant adjustments to be made.

\section{Querying the Contents of a Collection}

These are the methods in lines 8-11, Fig. 5.4. According to Naftalin and Wadler [49], the signatures of contains () and containsAll () in the original generified collection (lines 10 and 11, Fig. 5.3) are a more liberal alternative than the following:

boolean contains $(\mathrm{E} O)$;

boolean containsAll(Collection<? extends $E>C$ ) ;

The latter signatures catch more errors at compile time (while also ruling out some sensible tests). We could not adopt a liberal OGJ $J_{+}$version similar to that in line 7, Fig. 5.5, because $\mathrm{OGJ}_{+}$will not allow the method invocation in line 8 unless Owner (the owner of $c$ ) is inside the owner of $\circ$. Since the owner of $\circ$ is unknown, determining the nesting structure at compile time will not be possible. $O G J_{+}$can determine the nesting structure through the ownership information provided by the class header. $\mathrm{OGJ}_{+}$knows that the owner of the class must be inside all of the other owners involved in the class header. That is, using $\mathrm{E}$ as the type of $\circ$ means that Owner is inside the owner of $\mathrm{o}$. 


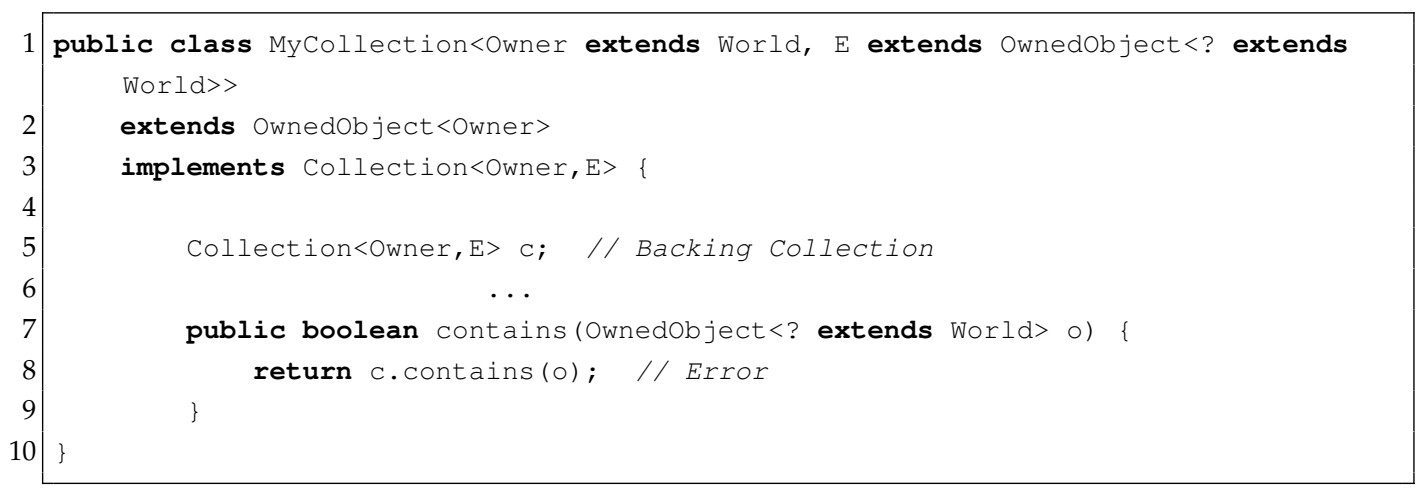

Figure 5.5: A liberal signature of contains ()

So, what applies to add() and $\operatorname{addAll}()$ applies to contains() and containsall(). The only difference is that containsAll() is owner-polymorphic. This is because the equals() method is ownerpolymorphic; see subsection 4.5 . The idea is that the implementation of the overriding equals (), in the abstract class Abstract Set, compares the specified Set (method parameter) for equality with the this set by checking if the this set containsAll() the elements in the specified set, after confirming that they both have the same size. So, to send a containsAll () message by passing the specified set as argument, containsAll ( ) needs to be able to infer the owner of the specified set, which is already inferred by equals ( ).

\section{Removing Elements}

These are the methods in lines 14-17, Fig. 5.4. The signature of remove () is treated in a similar fashion as the signature of contains (). Also, the signatures of removeAll () and retainAll () similarly correspond to the signature of $\operatorname{addAll}()$, although they utilise method genericity instead of using wildcards. Apart from the owner parameter, in the resulting byte code there is no difference between the signature of the generic removeAll () and its corresponding signature that uses a wildcard. We 
found the generic signature mandatory for our refactoring, however. The example in Fig. 5.6 shows the overriding removeAll () implementation for the collection class AbstractCollection of $O G J_{+}$. The method invocation in line 5 would not compile if the signature of removeAll () required its argument to be a Collection<Owner, ? extends $E>$, since contains () actual argument E cannot be converted to the capture of ? extends E by method invocation conversion. What applies to removeAll () applies straightforwardly to retainAll ().

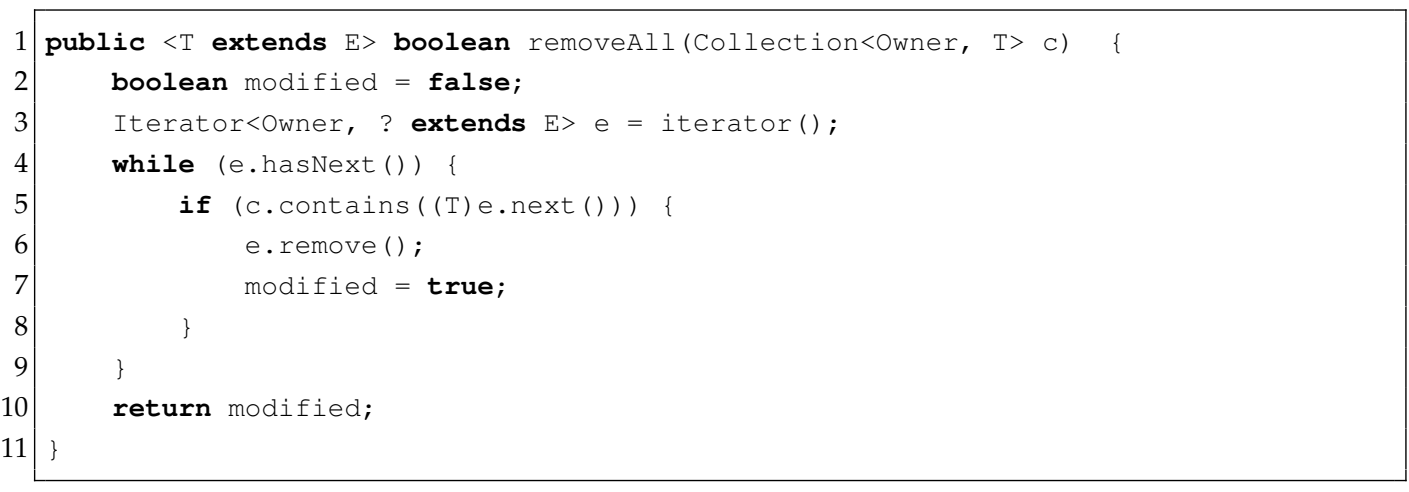

Figure 5.6: OGJ+AbstractCollection.removeAll ()

\section{Making a Collection's Contents Available for Further Processing}

These are the methods in lines 20-22, Fig. 5.4. The iterator() method returns an Iterator over the receiver collection. The two overloaded toOwnedArray () methods transfer the contents of the receiver collection into a new OwnedArray object. Both methods are the $O G J_{+}$versions of the two overloaded toArray () methods in the JDK 1.6 Collection interface. The advantage of the second toOwnedArray () method, line 22 , is that it infers the type of the returned array from the method's argument type in order to accurately realize control over the runtime type of the returned array. 
There are times when we need to transfer the contents of a sibling collection object into a private This-owned array object. For example, the ArrayList class has a constructor that takes a collection argument to construct a list containing the elements of that collection. Consider the example in Fig. 5.7 the formal parameter $c$, line 6, is owned by Owner, while the backing array object elementData is owned by This. That is, we cannot use an assignment statement such as elementData = c.toownedArray ( ) ; but we can have the returned OwnedArray owned by This, if toownedArray () is owner-polymorphic so that the assignment statement would appear as in line 7.

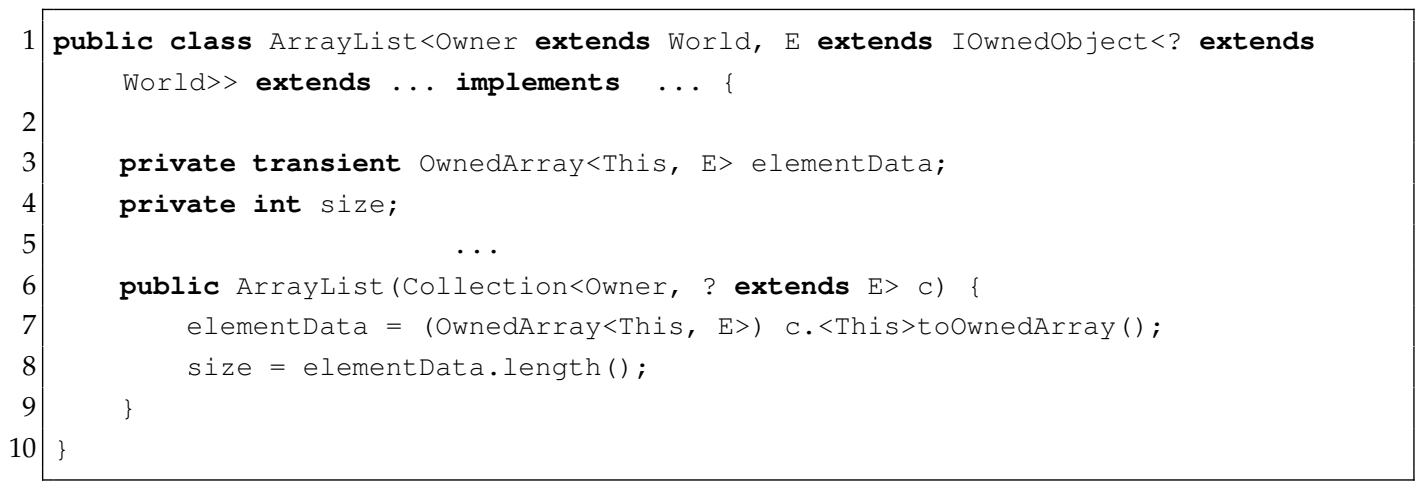

Figure 5.7: ArrayList constructor takes a Collection argument

The implementation of toownedArray () is one of the implementations that required more flexibility in handling the iterator () method's owner parametricity. That is, iterator() needed to be ownerpolymorphic in order to infer owner arguments that do conform to the owner arguments of other iterator-manipulating owner-polymorphic methods. 


\subsubsection{The Map Interface}

Map is the other dominant interface, in that it sets up the second interface hierarchy in the Collections Framework. Since any descendant of this interface represents a mapping object that maps keys to values, the header of this interface involves two type parameters: $\mathrm{K}$ for the type of the keys, and $V$ for the type of the values. Fig. 5.8 and Fig. 5.9 are the original JDK 1.6 and the $O G J_{+}$compliant versions of the interface Map, respectively. Unlike Collection, Map directly extends IOwnedObject, as it is not iterable; and as a matter of course, IOwnedObject preserves the same owner as Map. In close parallel to the categories of methods defined by Collection, the methods in Map can be grouped into: adding mappings, removing mappings, querying the contents of a map, and providing collection views.

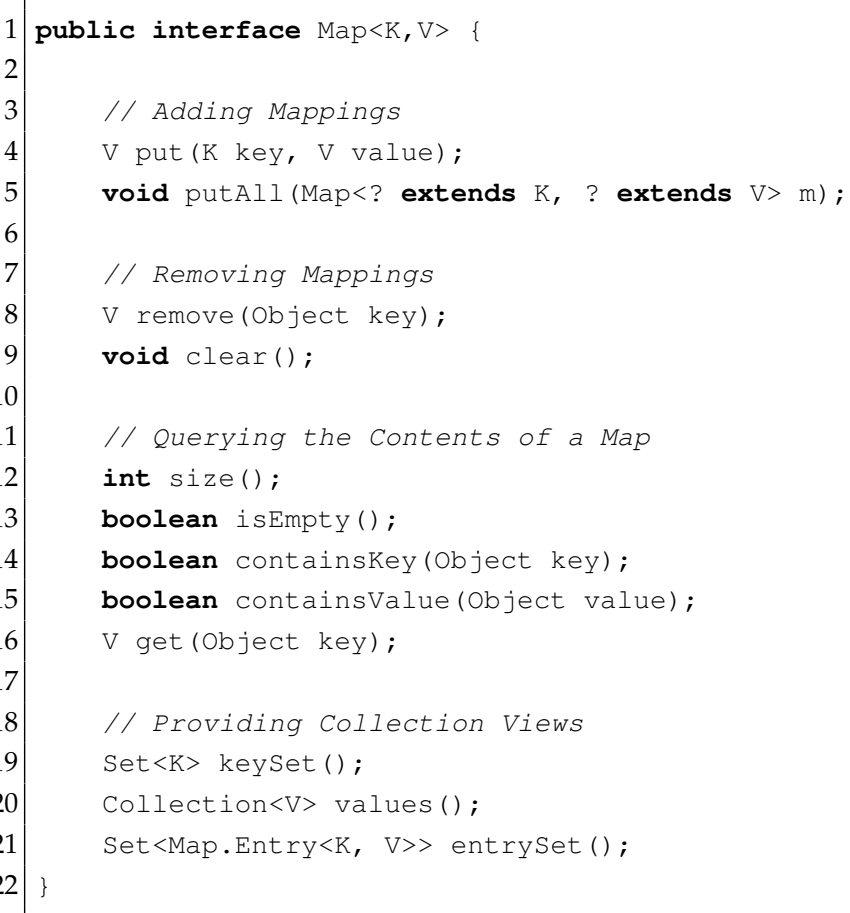

Figure 5.8: JDK 1.6 Map Interface. 


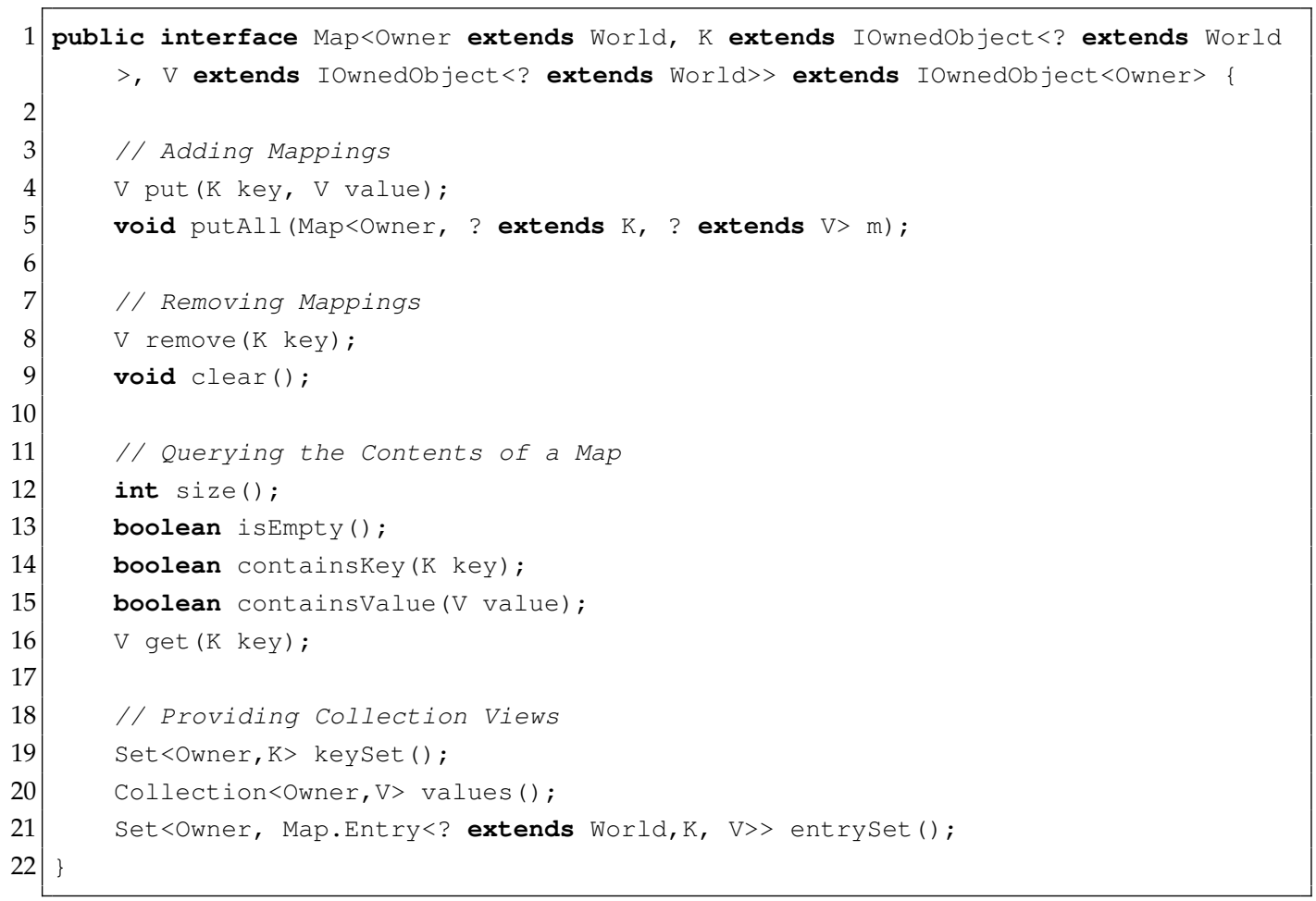

Figure 5.9: OGJ+ Map Interface.

As appears in Fig. 5.9, there is no significant changes in the first category; only putAll () needed to have an owner for the parameter type. Similar to contains (), in the previous subsection, the method signatures of the second and third categories are treated in a more preservative fashion than the original liberal version. As for the fourth group, since Map cannot explicitly be iterated over, this category provides three methods that return collection views, each with its own iterator. The first method keyset () returns a Set view of the keys. The second method values () returns a Collection rather than a set, due to the possibility of having duplicate values associated with different keys. The third method entryset () returns a set view of the mapping key $\Rightarrow$ value. The elements of the returned set implement the interface Map. Entry that embodies a mapping from a key to a value. Since the owner of the elements of any collection class, in our design, is subject to context covariance, the 
owner of the elements of the Set to be returned by entryset () is a wildcard.

\subsubsection{The Iterator Interface}

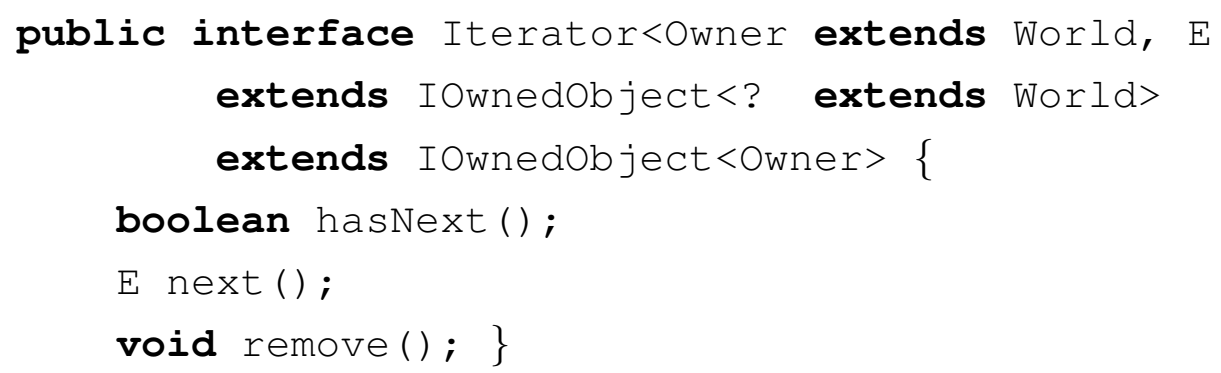

The Iterator interface is included in the package java.util together with the general-purpose collections. Any class that implements Collection maintains an iterator() method to return an Iterator object applicable to the instances of that class. The iterator () method is defined either inside the collection class itself or inside an abstract class (e.g., Abstract List) that provides a skeleton implementation of the relevant abstract data type (e.g., List).

Typically, iterators are implemented in the Collections Framework as inner classes implementing the Iterator interface; or as is the case with lists, iterators are inner classes that implement the interface ListIterator, which in turn extends Iterator. As clarified in the inner classes' subsection 4.2 , iterators are implemented as inner classes in order to maintain direct access to the private backing fields of the outer collection classes (and thereof to the element objects). The nesting between classes is not a nesting between objects, however; hence private fields are exposed to incoming aliases to inner classes' instances. Implementing iterators as inner classes is basically the main issue in applying ownership to collections. Backing fields, as the internal private representation of their containers, must be owned by This; and therefore, cannot be directly accessed even through a This-owned reference defined in an inner class. 
Consider the example of Fig. 5.10, header is a representation object of LinkedList, which means that header should be owned by This. ListItr is the iteration inner class of LinkedList. In the original implementation of LinkedList, header is assigned to lastReturned inside ListItr, as in line 7. If we are to make lastReturned owned by This, this does not mean that header and last Returned have the same owner. header belongs only to the outer class, and can only be accessed through the operations of LinkedList. Within the course of the rest of this chapter, we will demonstrate how each collection class treats its iterators.

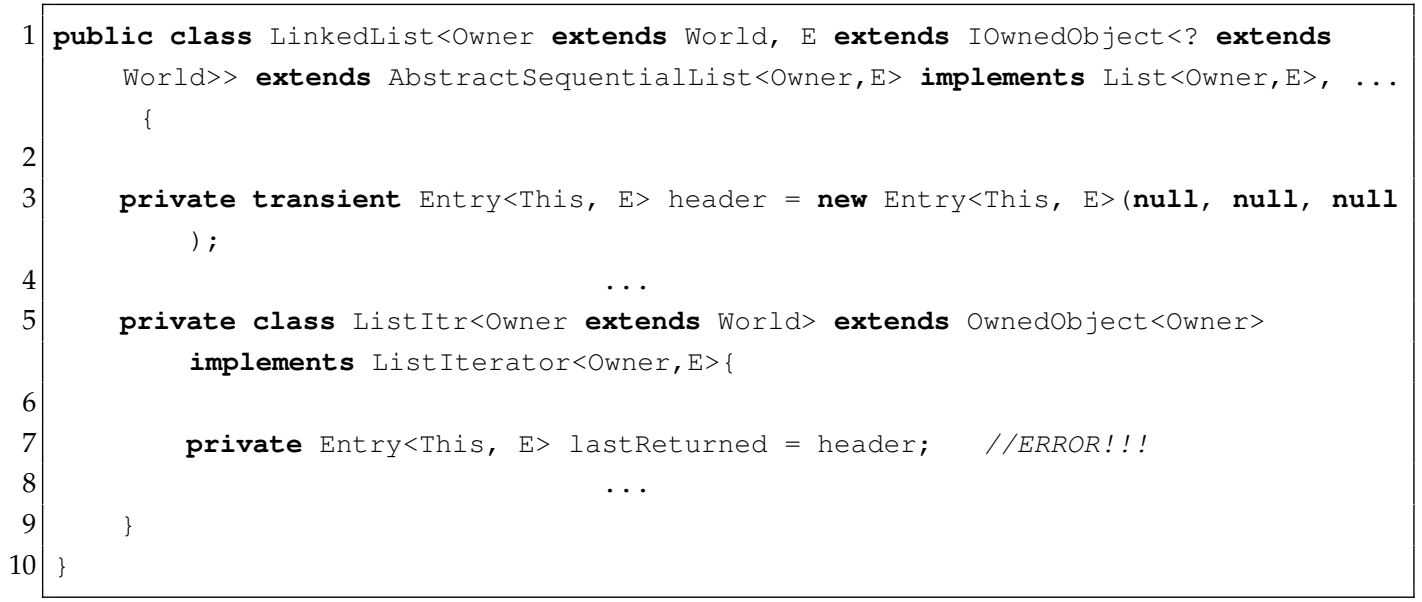

Figure 5.10: Linked List's Iterator as an Inner Class 


\subsection{Lists}

Lists are implemented through the interface List. List extends Collection and has a pretty similar header to Collection, see Fig. 5.11. Owner owns List, and $\mathrm{E}$ is the type of the elements.

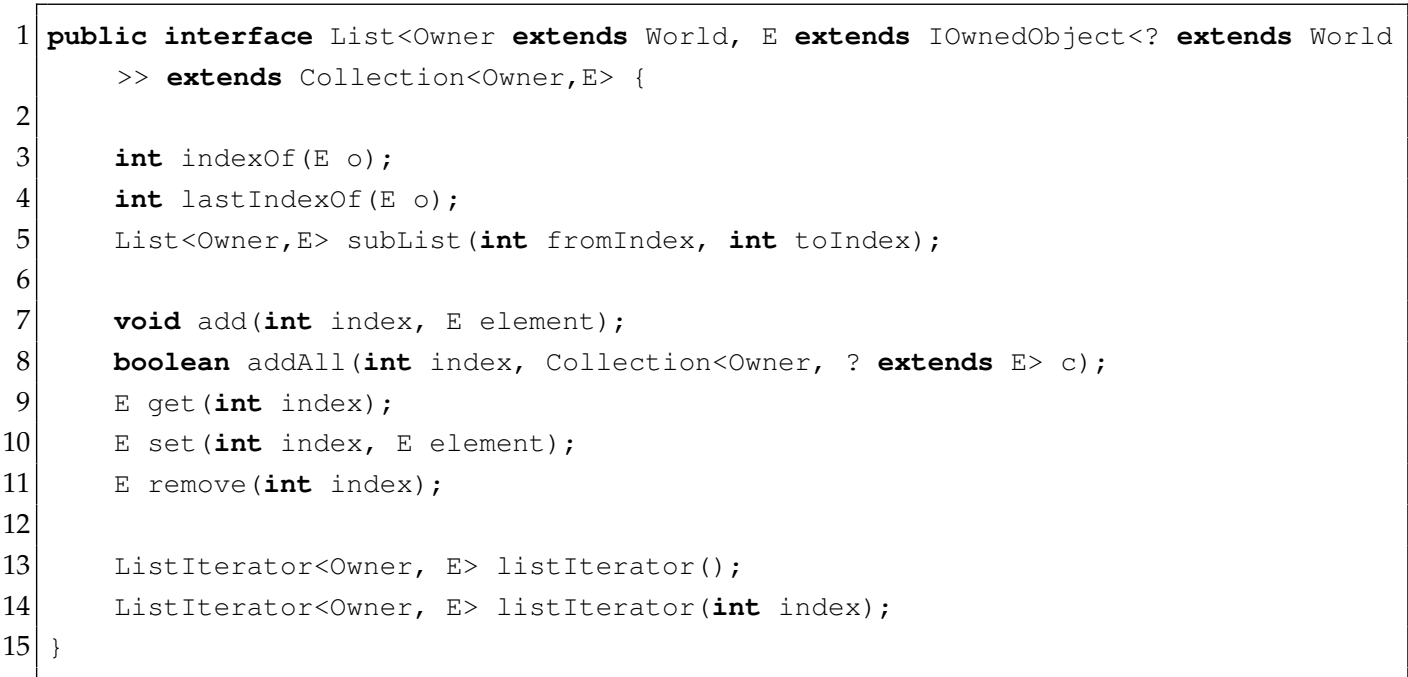

Figure 5.11: OGJ List Interface.

In order to provide search operations and range-views, List supports positional indexing so that the numerical position of a specified object (or a view of a range of the list) can be returned. The methods in lines 35, Fig. 5.11, support these operations. Since the nature of lists is to allow duplicates, List defines two methods for searching a specific element: the first one, in line 3 , is to return the position of the first occurrence of the specified element; the second, in line 4, returns the last occurrence. Method subList ( ), in line 5, returns a range-view of the receiver list preserving owners. The original signatures of methods indexOf () and lastIndexOf () involve Object as a parameter type, so they were adapted to be more preservative.

Together with the methods inherited from Collection, the List interface defines additional position-oriented methods for inserting (collec- 
tions as well as elements), retrieving, modifying and removing elements. These methods are in lines 7-11, Fig.5.11. Except for addAll (), the other signatures did not require any modifications. What applies to addAll () in Collection applies to the position-oriented version of $\operatorname{addAll}()$ in List.

Finally, there are two iteration methods (lines 13 and 14, Fig. 5.11); each of them is returning a ListIterator over the receiver list. The java.util.ListIterator interface extends the Iterator interface. Due to the sequential nature of lists, it is possible to have bi-directional access to a list; that is, traversing a list backwards and forwards. So, in conjunction with the three main operations of Iterator (namely hasNext (), next () and remove ()), List Iterator defines hasPrevious(), previous(), along with position-oriented methods such as next Index () and previousIndex (). Moreover, ListIterator defines add () and set () in order to facilitate insertions and replacements. All of these methods do not need any modifications in their signatures.

Back to the methods that return Listiterator, the first one is similar to iterator(), in Collection, in that it returns an iterator that is positioned at index 0 . The second method returns an iterator that takes its initial position at the specified index. During the implementation, we found no need to make these methods owner-polymorphic as collection.iterator(). We made them owner-polymorphic, however, so that their owner arguments can conform to the owner arguments of owner-polymorphic methods that might manipulate them.

\subsubsection{Array List}

ArrayList is a resizable version of an ordinary array. The ArrayList class is implemented as an array buffer in which the contents of the ArrayList are contained, and an integer field to keep track of the size of the list. These fields are referenced as elementData and size, respec- 
tively. That is, the representation objects of an ArrayList are those referenced as elementData and size. The field size is of value type int, and we have no aliasing problems with value types. As for elementData, the array field to which it refers is of type object, and it had to be substituted with an OwnedArray type. Since elementData is referring to a representation object, OwnedArray needs to be owned by This (i.e., to be private and hidden); which means that elementData can only be accessed via receiver this. Also, the type of the elements inside OwnedArray is no longer required to be other than $\mathrm{E}$ (originally elementData was of type Ob ject [ ] ), as we are no longer worried about Java's no generic array creation property, see subsection 4.1. The definition of elementData reads as follows:

private transient OwnedArray<This, E> elementData;

The ArrayList class provides no implementation for iterator() (defined in Collection) or for listIterator() (defined in List); instead, Arraylist inherits these implementations from the superclass Abstract List. Abstract List has two iteration inner classes; one implements Iterator, and the other implements List Iterator. Neither of these inner classes requires access to a representation field; AbstractList does not have fields. AbstractList.iterator () returns an implementation of the Iterator interface that depends entirely on three methods of the extending list (in this case ArrayList). These methods are size(), get (), and remove(). Likewise, AbstractList.listIterator() returns an implementation of the List Iterator interface that depends on the get (), set (), add (), and remove () methods of Arraylist. Arraylist encapsulates its representation, and thus the adaptation of ArrayList and AbstractList is just to make them compliant with $\mathrm{OGJ}$. 


\subsubsection{Linked List}

LinkedList is the other concrete implementation of List in this study. In contrast to ArrayList, which preserves encapsulation by depending entirely on the List interface, LinkedList preserves efficiency by having an iterator that bypasses the interface through an incoming reference to the private representation of LinkedList. That is, efficiency is preserved by breaching encapsulation. As depicted in Fig. 5.12, the entry nodes of the list are a number of objects placed into the heap with each of them having its own data plus pointers to the next and previous nodes; then, a List Iterator, as an outsider object, bypasses the List object's interface operations, and directly accesses the list's entries by pointing at any object in the chain.

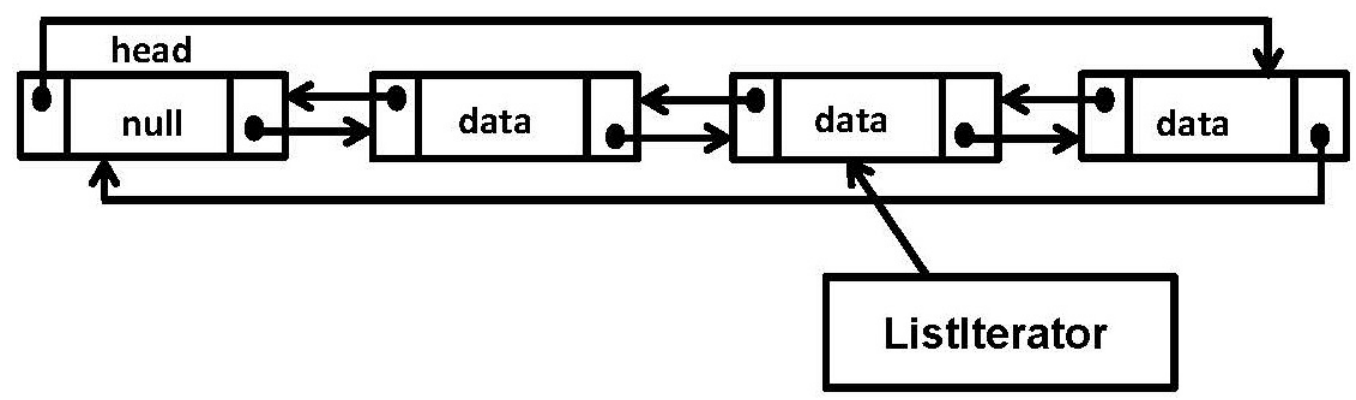

Figure 5.12: Linked List with Iterator

An entry node is implemented in LinkedList as a static member class called Entry, and contains three fields: element, next, and previous. LinkedList uses a list header that has the same formation as any other entry node. That is, to construct a new empty linked list, a new header object should be instantiated with a null element and pointers to itself, then new entry nodes can be linked to that header or inserted between each other. The LinkedList class is implemented as to have two fields: an Entry object referenced as header, and an integer size to keep track of the size of the list. These are our representation objects; and thus, header should be owned by This. The example of Fig. 5.13 shows the declara- 
tions of the fields and constructors.

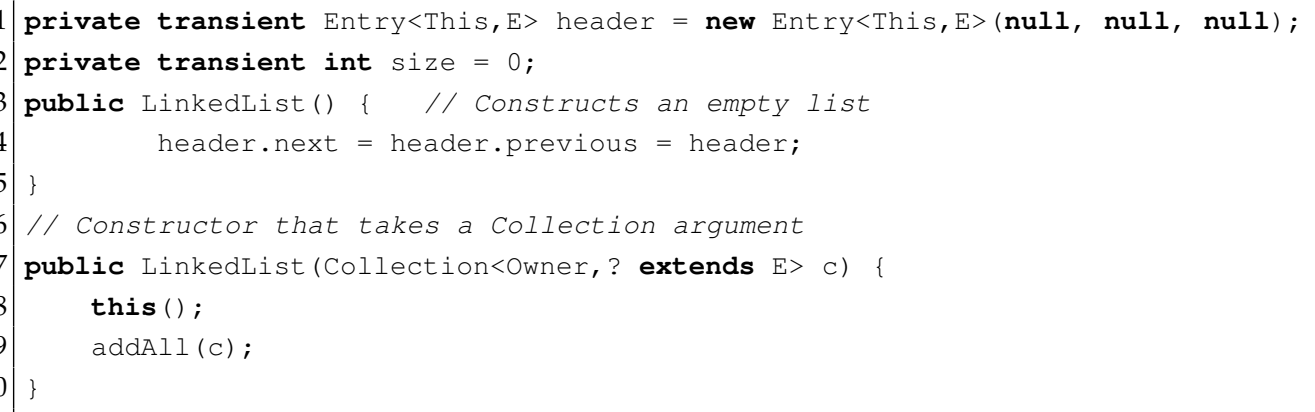

Figure 5.13: LinkedList Fields and Constructors

Similar to ArrayList, LinkedList inherits the implementation of Collection.iterator() from AbstractList to return an iterator for traversing the list from head to tail and safely removing entries. LinkedList also provides an implementation for Deque. descendingIterator ( ) to return an iterator for traversing the list from tail to head and remove entries. That is, LinkedList.descendingIterator() returns an implementation of the Iterator interface; this implementation is an inner class of LinkedList and does not maintain any incoming aliases as it provides iterators via previous () and hasprevious() methods of the inner class implementation of List Iterator.

As explained earlier in subsection 5.1.3, ListItr is the inner class implementation of ListIterator in LinkedList. As the example in Fig. 5.10 shows, the representation object header is assigned to lastReturned when the iterator is initially constructed. What happens is that lastReturned gets information from the other incoming reference next that points to the current Entry node. Methods next () and previous () return lastReturned after setting it to next that moves along the linked nodes forward and backward by these methods. This will still be the case with LinkedList as an OGJ + compliant implementation, since the incoming references from List Itr to the internal representation 
of LinkedList are wildcard-owned, and hence readonly.

ListIterator supports adding, removing and changing elements in the underlying list. The implementations of add (), remove (), and set () in LinkedList. ListItr is made through the operations of the LinkedList, and thus there were no significant changes required to preserve encapsulation in accordance with the rules of $O \mathrm{OJ}_{+}$. 


\subsubsection{Vector (legacy)}

Vector is the legacy version of ArrayList. The main difference between a Vector and an Arraylist is that the Vector class is synchronized. Vector has exactly the same internal representation as ArrayList. Also, Vector inherits the implementations of Collection.iterator() and List.listIterator() from AbstractList, where the returned iterators are implemented as inner classes. So, in relation to these implementations, Vector maintains the same encapsulation guarantees as ArrayList.

Additionally, Vector provides the method elements () that returns an implementation of the legacy interface Enumeration. Enumeration iterates over the implementor collection for retrieval of stored elements. Enumeration is implemented in Vector as an anonymous class. An anonymous class can be declared in $O G J_{+}$as a sibling of the enclosing class. That is, the instances of Vector and the sibling implementation of Enumeration are located in the same ownership context, but Vector and its internal representation form another nesting context, as depicted in Fig. 5.14. Accordingly, Enumeration has no right to directly access the internal representation of Vector. The example in Fig. 5.15 shows how the Enumeration interface can be implemented as an anonymous class owned by the same owner of as Vector

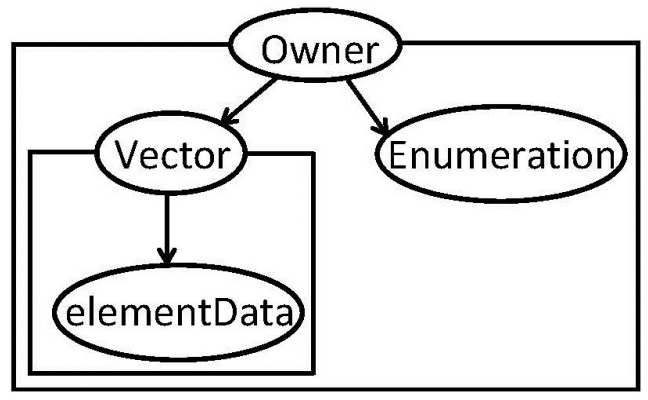

Figure 5.14: An object of an anonymous class as a sibling of the enclosing object 


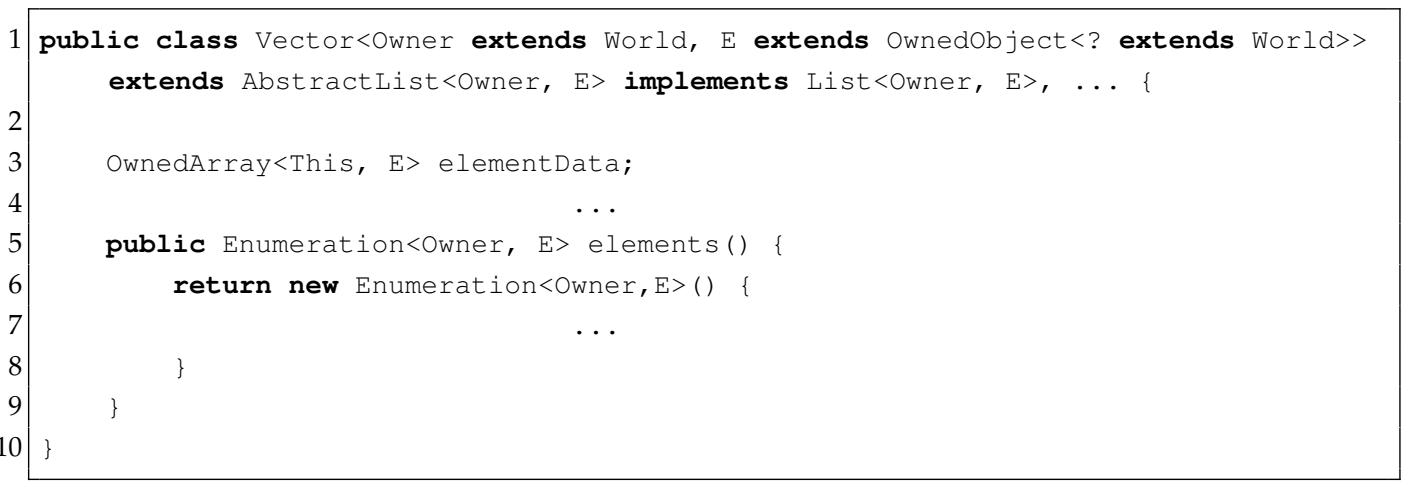

Figure 5.15: Enumeration implemented as an anonymous class in the same context as Vector

Enumeration provides two methods (hasMoreElements() and nextElement ()) for traversing a Vector object. Method nextElement () returns the element at the relevant position by directly accessing the backing array, which means that the elements can be retrieved without the Vector's knowledge. To correct this breach, that $O G J_{+}$does not allow, nextElement () was modified to depend on the get () methed of the current Victor object.

\subsection{Queues}

Applying generic ownership to the interfaces Queue and Deque (doubleended queue) is no different to the interfaces explained above. All modifications can be justified on the premises mentioned in subsection 5.1.1. This section describes the issues addressed in two implementations of these interfaces. PriorityQueue implements Queue; and ArrayDeque implements Deque.

\subsubsection{Priority Queue}

Priorityqueue is backed by an array. The representation objects of this 
implementation are quite similar to those of Arraylist and Vector: an array buffer to hold the contents of the queue; and an integer for keeping track of the size of the queue. This implementation maintains its iterator as an inner class, as mostly the case with collections; and as commonly happens, a next () method directly accesses the backing array. In Vector, we modified nextElement () to depend on the get () method, which is defined within the body of the Victor class. Due to the FIFO nature of queues, the Queve interface does not provide any position-oriented methods for retrieving elements form the backing array. To overcome this, we defined a private method within the body of PriorityQueue to get the element at the specified index, and made next () rely on this private method.

\subsubsection{Array Deque}

As its name implies, the ArrayDeque implementation is also backed by an array. Since a deque supports insertion, retrieval, and removal of elements at either ends of the queue, ArrayDeque has two representation integer fields, namely head and tail, to keep track of the indices of the elements at the head and tail of the deque. ArrayDeque is no different to Priorityqueue, however, in that it does not provide element retrieval via position-oriented methods.

This implementation maintains two iterators, namely DeqIterator and DescendingIterator, also as inner classes. Each of these iterators has a next () method to return the element at the cursor position. Both methods directly access the backing array on two occasions: first, to get the relevant element; and second, to get the length of the array in order to calculate the next cursor position. Moreover, the remove () method in both iterators accesses the array to get its length in order to recalculate the cursor position, so that the increment made by next () can be undone. In a similar fashion to PriorityQueue, both iterators were refactored to 
relay on two private methods defined within the body of ArrayDeque; one to return the element at the specified index, and the other to return the array length.

\subsection{Maps}

Although a map can be thought of as a special kind of set of values associated with unique keys (one-directional mapping from a key to a value) the Map interface does not extend Set or Collection. On the other hand, the set interface general-purpose implementations are backed by Map implementations in order to make use of the properties of these Map implementations. For example, the Hash Set implementation does not provide a specific Iterator; instead, the method Hashset. iterator () returns an iterator over the key set view of the backing HashMap. Since it is not possible to iterate over a Map object directly, Map provides set views. That is, the relationship between the notions of sets and maps forms a key element in their design and implementation. So, why does Map not extend Collection? Further, why does set not extend Map? The answer to these questions are best described in Java Collections API Design FAQ [2]:

"This was by design. We feel that mappings are not collections and collections are not mappings. Thus, it makes little sense for Map to extend the Collection interface (or vice versa). If a Map is a Collection, what are the elements? The only reasonable answer is "Key-value pairs", but this provides a very limited (and not particularly useful) Map abstraction. You can't ask what value a given key maps to, nor can you delete the entry for a given key without knowing what value it maps to. Col lection could be made to extend Map, but this raises the question: what are the keys? There's no really satisfactory answer, and forcing one leads to an unnatural interface. Maps can be viewed as Collections (of keys, values, or pairs), and this fact is reflected in the three "Collection view operations" on Maps (keyset, entryset, and values). While it is, in principle, possible to 
view a List as a Map mapping indices to elements, this has the nasty property that deleting an element from the List changes the Key associated with every element before the deleted element. That's why we don't have a map view operation on Lists.".

So, in terms of design, conformance, and coding, treating maps as Collection implementations (i.e., Map extends collection) would definitely hinder the flexibility of implementing maps, as well as the performance, resulting in an inefficient Map abstraction. Treating collections as Map implementations is not reasonable in terms of handling the contents, properties, and solution design options. Maps are set containers, however; mathematics says so, and even the code says so.

The Collections Framework provides three general-purpose Map implementations: HashMap, LinkedHashMap, and TreeMap. This study is concerned with these implementations of Map, the legacy implementation HashTable, and the special-purpose implementation IdentityHashMap.

\subsubsection{Hash Map}

HashMap is a Map implementation backed by an array. The contents of this array are the mapping entries of the table that makes up the internal representation of HashMap. Each mapping entry is an instance of the static inner class HashMap. Entry. In the List and Queue implementations, the routine was to hide the backing OwnedArray objects by making them owned by This, since they hold the elements. In LinkedList, the Entry objects, which each hold an element, needed to be hidden, since they are consistently available as entry points. With HashMap we need to hide both the backing array as well as the Entry objects that hold the key-value mappings. So, the backing field table would be declared as follow:

OwnedArray<This, Entry<This, K, V>> table;

As explained in subsection 5.1.2. Map provides methods that return iterable collection views. These methods are keyset (), values() and 


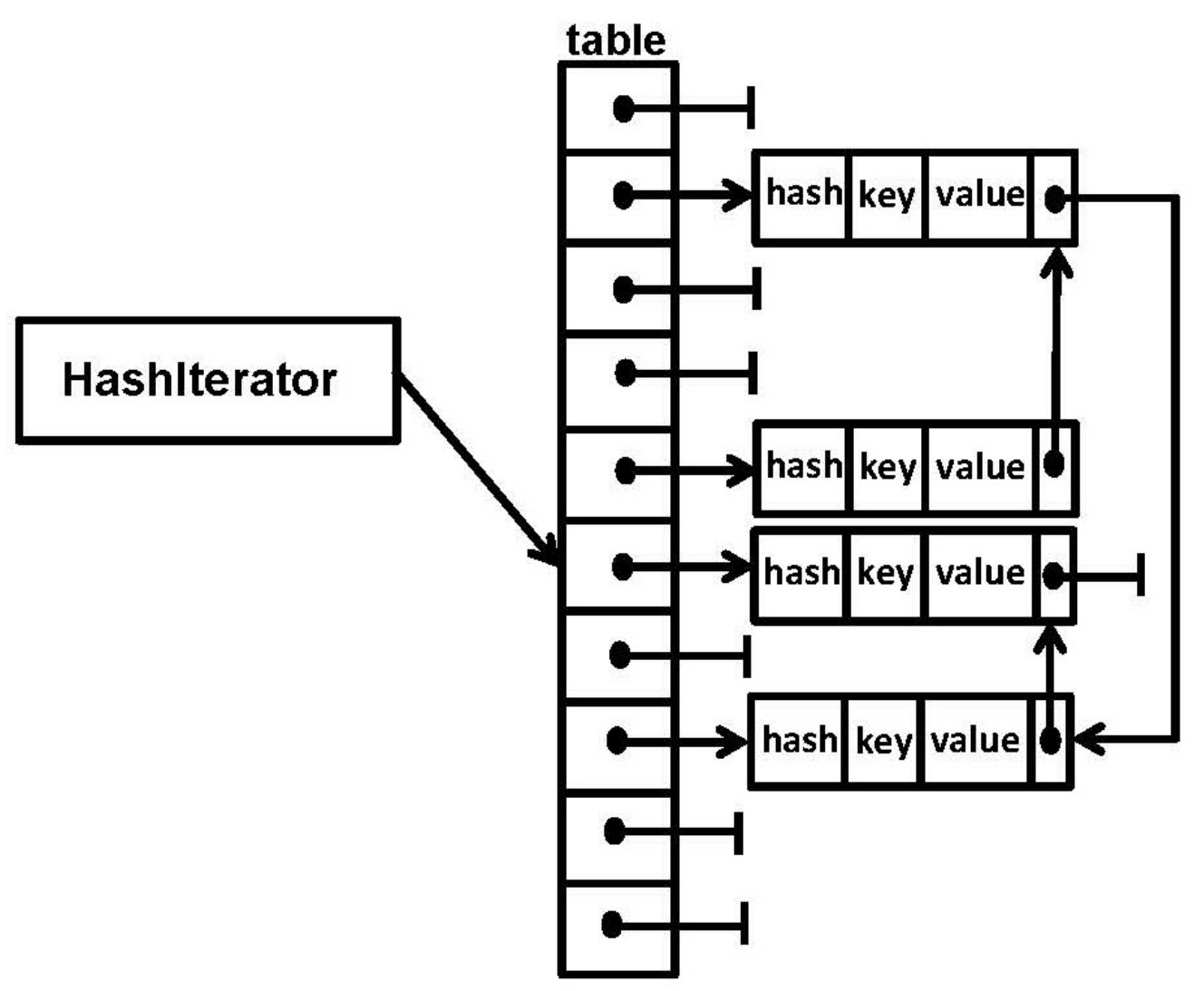

Figure 5.16: Hash Map with Iterator

entryset (); their signatures appear in Fig. 5.9. The views are backed by the current map; that is, changes in the map are reflected in the views, and vice-versa. To maintain this mirroring property while allowing each view's iterator to support entry removal, each iterator needs to run over the indexed mapping entries of the backing array. In doing so, HashMap provides three iteration inner classes, each for a view, as adapters that provide iterators via HashIterator. nextEntry (). HashIterator is a private abstract inner class implementation of the Iterator interface, and does not override the abstract method Iterator. next (). Each of the three adapters extends HashIterator in order to override Iterator.next() in behalf of HashIterator. Each adapter's 
overriding next () relies on the indexed mapping entry returned by the nextentry () method. The nextentry() method and the constructor of HashIterator maintain incoming references to table. Through these reference, nextEntry () accesses table to get its length, and runs another pointer, next, over the table entries to get, and return, the Entry object at the specified index.

To refactor this breach, we removed the incoming reference to table, and kept next as a wildcard owner parameterised reference, so that next can point to the Entry objects which are owned by This (i.e., the this HashMap). Since accessing the table needs to be done through the HashMap operations, we need to find methods that return indexed mapping entries and the table length. HashMap has a capacity () method which returns the length of the backing array. HashMap treats table as indexed by keys, and hence has no methods that allow dealing with table as indexed array. We defined a private method inside HashMap to return the Entry object at the specified index, and made next point to the returned entry.

\subsubsection{Linked Hash Map}

The LinkedHashMap implementation inherits from HashMap. LinkedHashMap maintains a doubly-linked list over the mapping entries to be stored in HashMap.table. LinkedHashMap. Entry inherits from HashMap.Entry, and provides additional pointers to the next and previous Entry objects to form the required nodes for a linked list. Similar to LinkedList, LinkedHashMap uses a list header, which is an entry node with a null mapping. That is, an Entry object referenced as header is our representation object that should not be accessed via incoming aliases. 
The collection views' iterators are implemented in LinkedHashMap in the same fashion as in HashMap. The views' iterators inherit from the private abstract inner class LinkedHashIterator, which correspond to HashIterator in HashMap. LinkedHashIterator maintains an incoming reference to the representation field header. This reference must be owner parameterised with a wildcard, so that it can safely be set to header.

Since lookups are based on unique keys, the implementation of HashMap uses a hash to store the key in the map for fast lookups. Therefore, a HashMap does not guarantee the order of the mapping entries. By maintaining a doubly linked list, LinkedHashMap maintains the order of the entries according to the order in which they are inserted. Moreover, LinkedHashMap can maintain the ordering according to the order in which the entries are accessed. In doing so, LinkedHashMap provides a constructor that takes a boolean parameter to switch between the ordering modes. This boolean parameter is then assigned to the representation field accessorder. Apart from this constructor, for every constructor declared in HashMap, LinkedHashMap declares a corresponding constructor. All of the constructors of HashMap are invoked by super() in their corresponding constructors of LinkedHashMap, and accessorder is set to false. In order for LinkedHashMap to initialize the header node, the Template Method design pattern is adopted. This pattern requires two kinds of methods, one is called a template method, and the other is called a hook (or placeholder) method. The template method (in a superclass) contains the invariant behaviour and invokes the hook (in a subclass) for the variant behaviour. All of the constructors of HashMap are template methods that call a hook called init (). Method HashMap.init () does not hold any behaviour on its own, while the overriding LinkedHashMap.init () initializes the header node of the linked list. When the constructors of LinkedHashMap call super (), the header node is initialised via LinkedHashMap. init (). 
In a similar fashion, methods put() and putForNullKey() are implemented in HashMap as template methods that invoke the hook recordAccess () which is declared in HashMap. Entry. This hook contains no behaviour. The overriding hook recordAccess () of LinkedHashMap. Entry moves the receiver mapping entry to the end of the list, if LinkedHashMap is access ordered (i.e., access 0 rder flags true). In doing so, recordAccess () inserts the entry before the header. That is, recordAccess () needs to access both accessorder and header. Class Entry is a static inner class from which non-static variables cannot be referenced. That is why recordAccess () takes a HashMap parameter. This parameter is then assigned to a LinkedHashMap local variable, through which the representation fields can be accessed; see the example in Fig. 5.17 .

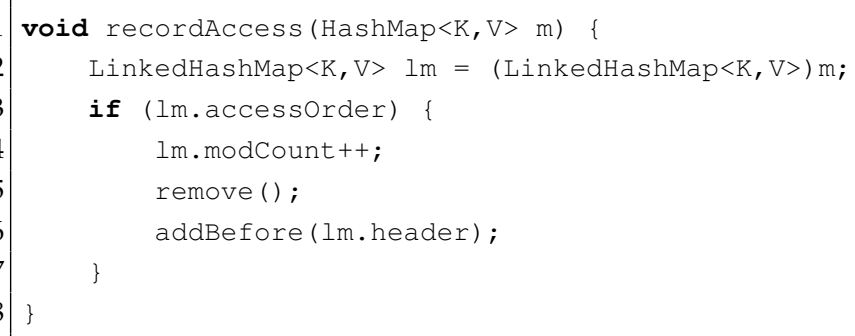

Figure 5.17: Java 1.6 LinkedHashMap. Entry . recordAccess ()

$\mathrm{OGJ}_{+}$does not permit the field access in line 6, Fig. 5.17, since header is owned by This. To overcome this restriction, we defined a private getter method inside LinkedHashMap to return a wildcard owner parameterised reference to the first entry, so that the statement in line 6 reads as addBefore(lm.getFirstentry (). before). This required addBefore () to take a parameter of an unknown context, and to make the entry nodes' pointers (after and before) also wildcard owner parameterised. This solution worked for the above mentioned conjunction between the static context and the template method pattern. 
The OGJ approach to wildcards did not work, and should not, for a hook such as transfer (), whose behaviour is to move the contents of the backing array to another resized array. The resizing template method declared in HashMap assigns the new array object to table after invoking transfer(). This means that the new resized array and its entry objects should be owned by This. The overriding transfer() of LinkedHashMap makes use of the linked list for faster iteration. If after and before are of unknown context, then we cannot assign them to entry objects owned by This. We decided to keep the wildcard solution for recordAccess () and rely on the transfer () method for HashMap.

\subsubsection{Hash Table (legacy)}

Hashtable is the legacy version of HashMap. The main difference between Hashtable and HashMap is that Hashtable is synchronized. Hashtable maintains similar representation objects as HashMap. That is, Hashtable is a Map implementation backed by an array. The mapping entries of the backing array, table, are instances of the private static class Entry. The definition of table reads exactly as that of HashMap; OwnedArray is owned by This, and the Entry objects should also be owned by This.

Hashtable extends the abstract parent Dictionary, which provides the methods keys () and elements () that return enumerations of the keys and values, respectively. The returned enumeration objects are implementaions of the legacy interface Enumeration. This way, Dictionary provides methods for traversing the keys and values, but not the entry mappings; and Enumeration traverses a Hashtable object without modifications. Hashtable provides the private inner class Enumerator which implements both the Enumeration and Iterator interfaces. This way, Enumerator can traverse a Hashtable object as a dictionary (associative array) and as collection views. Hashtable pro- 
vides key set, value collection, and entry set views, but none of them has its own implementation of Iterator. Enumerator has a constructor that takes two parameters; an integer parameter and a boolean parameter. Enumerator serves as an iterator if the boolean argument is true. The integer argument can be set to 0,1 , or 2, for traversing keys, values, or entries, respectively. Apparently, there is no use for an Enumerator such that: new Enumerator(2, false).

The Enumerator.next () method (overrides Iterator.next ()) relies entirely on the Enumerator.nextElement () (overrides Enumeration.nextElement()). The overriding next() method calls nextElement () and returns the value that nextElement () returns. The return type of nextElement () is of type $\langle\mathrm{T}\rangle$, which is declared in the header of Enumerator as the type of elements, and extends IOwnedObject<? extends World>. Method nextElement() returns an element such that:

type $=0$ ? $(T)$ e.key : (type $==1$ ? $(T)$ e.value : $(T)$ e)

Variable type denotes the value of the constructor integer argument, while $e$ is a wildcard owner parameterised reference to the current Entry object which is owned by This. OGJ ${ }_{+}$accepts the first two casts, since the owner of the type variable ( $\mathrm{K}$ for keys, or $\mathrm{V}$ for values) is the same owner of the elements that the Enumerator instance is to traverse over. In fact, Enumerator is to be instantiated using the very same type variable (K or V). Per contra, there is no way to allow the third cast in $O G J_{+}$. Although it is possible to assign an object owned by This to a readonly wildcard owner parameterised reference, it is not possible to allow a cast using incompatible owners. Therefore, we had to provide a special implementation of Iterator for the entry set views. 


\subsubsection{Identity Hash Map}

Map's general contract mandates the use of the equals ( ) method to determine equality using the state of the objects. In violation to this contract, IdentityHashMap performs identity-equality comparisons to determine uniqueness. A key is considered equal to another key only if they are references to the same object. Since the object state is disregarded, an IdentityHashMap object can contain key values and mappings that would be dealt with as duplicates in other Map implementations. In addition to this distinct property, IdentityHashMap is also different in that it uses a technique called hashing with linear probing [53]. This technique is used to deal with hash collisions of values returned by the hash () method. IdentityHashMap uses this technique to sequentially search the hash table for a free pair of adjacent locations, since IdentityHashMap stores the key and value references directly in adjacent locations in the table, not in entry cells; the key is placed at table[hash], while the value is placed at table[hash+1]. That is, IdentityHashMap does not use Entry objects, and hence the backing field table would be declared as follows:

OwnedArray<This, IOwnedObject<? extends World>> table;

In addition to the key set and value collection views, IdentityHashMap provides entry set views, however. Since IdentityHashMap does not maintain entry cells, the iterator of the entry set view is itself used as an entry that extends IdentityHashMapIterator and implements Map. Entry. IdentityHashMapIterator is similar to HashIterator and LinkedHashIterator in that it is an abstract inner class implementation of the Iterator interface, and does not override the abstract method Iterator.next (). The methods which override the abstract methods of Map. Entry rely on an incoming reference, defined in IdentityHashMapIterator, to table. This reference had to be owner parameterised by a wildcard in order to safely be set to the This-owned table. 
Each view iterator overrides Iterator. next () relying on the indexed key returned by IdentityHashMapIterator.nextIndex (). Determining the initial index ( 0 if table is not empty, or table length if table is empty) requires access to table to get its length. That is, an incoming reference to table is maintained. To maintain access to table through the IdentityHashMap operations, we defined a private method within the body of IdentityHashMap to return the table length, and made the iterator depend on this method.

\subsubsection{Tree Map}

The Collections Framework of Java 1.6 has the new interface NavigableMap. The NavigableMap interface is a subtype of the SortedMap interface. TreeMap is the only general purpose collection that implements NavigableMap. As an implementation of NavigableMap, TreeMap provides navigation methods such as descendingkeyset (), descendingMap (), headMap (), tailMap () and subMap (). That is, TreeMap provides more views than the ordinary views (i.e., Keyset, Values and Enteryset); for example, AscendingEntrySetView, DescendingEntrySetView, AscendingSubMap, DescendingSubMap; each has its iterator. A lot of operations are involved; submaps and entries are defined more precisely; as a result, many different inner classes (static and nonstatic) had to be involved.

Except for LinkedList, all of the Collection and Map implementations that have been studied up to this subsection are backed by arrays, and we used to make the backing arrays owned by This. Except for Ident ityHashMap, all of the other hash-based Map implementations use entry cells to hold the mappings, and these cells had to be owned by This. With LinkedList and LinkedHashMap, the list header is fixed and has a null element or null mapping. With TreeMap, the root is neither fixed nor having a null mapping. The root is part of the tree and is the mid- 
dlemost element. The mapping entry that represents the root of the tree could be swapped through right and left rotations as we perform add and remove operations. That is, the backing field of TreeMap, namely root, should always be available to be set to a mapping entry. If we are to have root owned by This while changing the reference structure through rotations, then all of the references to the entry nodes which are involved in a rotation operation must be owned by This and declared within the same class as root. Because of the many different inner classes involved in the implementation of TreeMap, and because the entry nodes must be accessed through the operations of TreeMap, inner classes need to send messages to TreeMap by passing references to entry nodes as arguments. These references had to be owner parameterized by wildcards, and most of the operations of TreeMap had to be able to accept argument types that have wildcards as owner parameters. That is, root needs to be owner parameterized by a wildcard in order for the rotation operations to set root to mapping entries. Since all entry nodes must be owned by This, we made sure that every new entry node is initialized within an insertion method as follows:

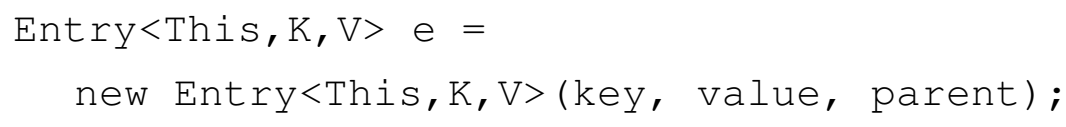

Since all of the entries that make up the internal representation of TreeMap is to be initialized owned by This, we can safely declare the backing field root as follows:

private Entry<? extends World, K, V> root;

\subsection{Sets}

Sets are implemented through the interface Set. Set extends Collection, overrides all of the methods of collection, and does not provide more methods than collection does. This study is concerned with three implementations of Set, namely HashSet, LinkedHashSet and 
Treeset. These sets are backed by Map implementations, and can be iterated over through the key iterators of their respective backing maps. The implementation of these sets is quite simple, and hence the refactoring is quite straightforward.

\subsubsection{Hash Set}

HashSet is backed by a HashMap instance referenced as map. HashSet relies only on the key store maintained by the backing HashMap to prevent duplicates. All mapping values are set to dummy values of type ob ject.

Since we made sure that the backing table of HashMap and the contained entry cells are hidden, then the elements of Hashset (i.e., the keys of the backing map) are also hidden whatever the owner of the backing field map is. We made map owned by This, however. Since the signature of Set.iterator () is owner polymorphic, the overriding method Hashset.iterator () can properly return the type returned by the call map.keyset (). iterator (). The backing field map is declared as follows:

HashMap<This, E, IOwnedobject<? extends World>> map;

The type argument of map's keys is $\mathrm{E}$, which is the type of HashSet's elements; and the type argument for the dummy values is the $O \mathrm{OJ}_{+}$'s equivalence of object.

\subsubsection{Linked Hash Set}

The LinkedHashSet implementation inherits from HashSet. The LinkedHashSet class has no backing field and has only four constructors; no other operations are defined in this class. All of the operations that a LinkedHashSet instance can conduct are inherited from HashSet. The four constructors of LinkedHashset invoke only one constructor from HashSet. The invoked constructor form HashSet is made especially for LinkedHashSet and instantiates LinkedHashMap, so that the opera- 
tions of HashSet can be conducted on a LinkedHashMap instance rather than a HashMap instance. Therefore, there are no security issues with this implementation.

\subsubsection{Tree Set}

In the same manner as described for TreeMap, Treeset is the only general purpose collection that implements the Navigableset interface. Navigableset is a subtype of the SortedSet interface. That is, in addition to the sorting mechanisms, Treeset provides the same navigation mechanisms as that of TreeMap, but only on the key store of the backing TreeMap. The backing field of TreeSet is of type NavigableMap, however. This is mainly because some of the navigation methods of TreeMap return NavigableMap views, and the corresponding navigation methods in Treeset are made simple as to return new instances of Treeset backed by these NavigableMap views.

As described for HashSet, there is no need to make the backing field owned by This since the elements are properly encapsulated in the key store of the backing map. We made the backing map of Hashset owned by This, and this did not cause any owner compatibility problems. For Treeset, we decided to avoid making the backing map owned by This in order to avoid any owner compatibility issues between Treeset and TreeMap. The backing field is declared as follows:

NavigableMap<Owner, E, IOwnedObject<? extends World $>>\mathrm{m}$;

The backing map is owned by owner which denotes the owner of Treeset. The type argument of the keys is $E$, which is the type of TreeSet's elements; and the third type argument is for the dummy values. 


\subsection{Usability}

Using the refactored collections requires a significant programming overhead, as appears in Fig. 5.19 which is the OGJ,'s version of Fig. 5.18. Although $O \mathrm{OJ}_{+}$provides stronger encapsulation guarantees, it is hard to believe that productivity will not decline using our version of the Collections Framework. Using $\mathrm{OGJ}_{+}$, and in particular the refactored collections, requires increased effort since, in addition to the annotation overheads, programmers will have to deal with the fact that standard code reuse practices became entangled by the use of deep ownership types.

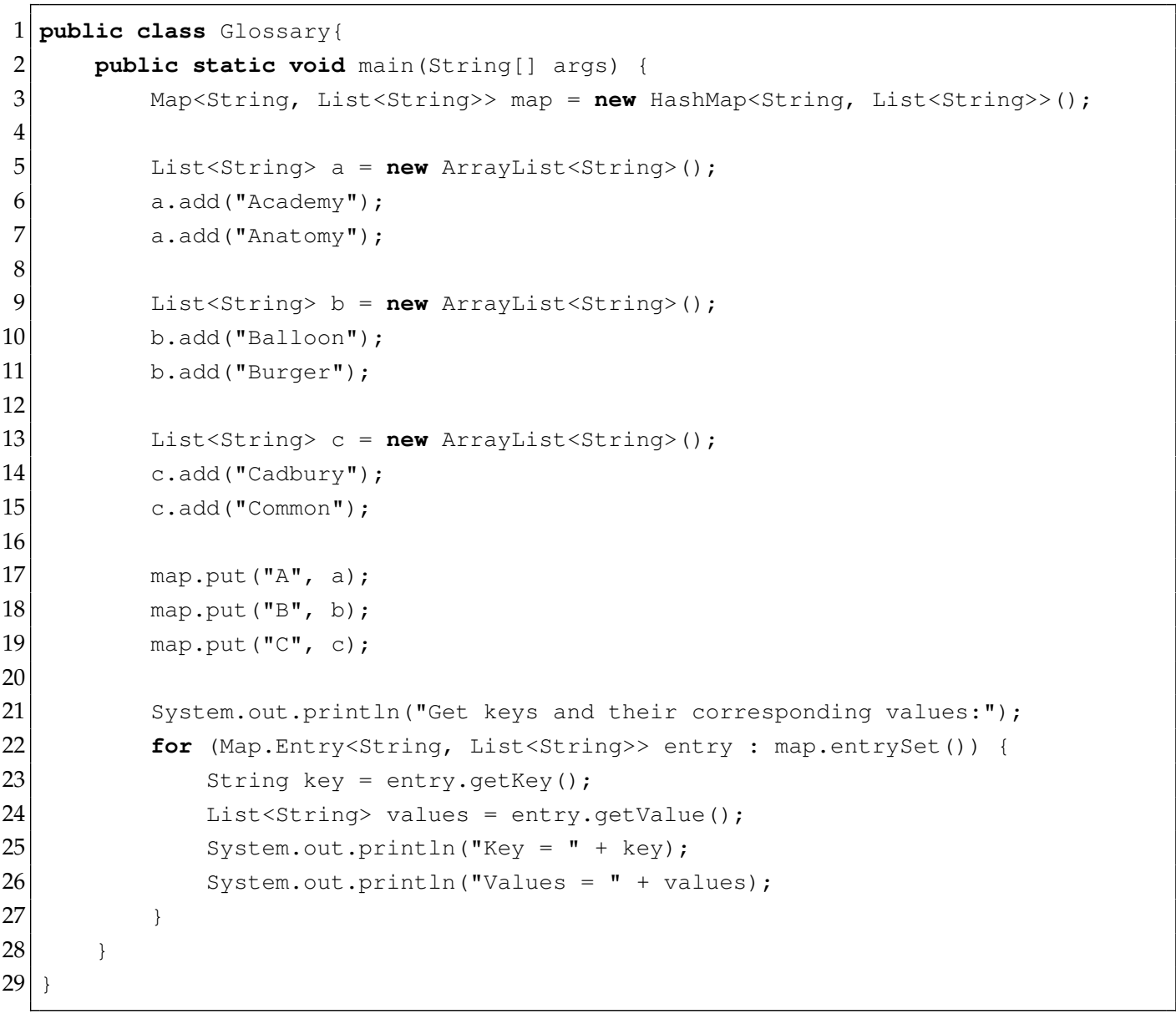

Figure 5.18: A HashMap storing multiple values for the same key using Arraylist. 


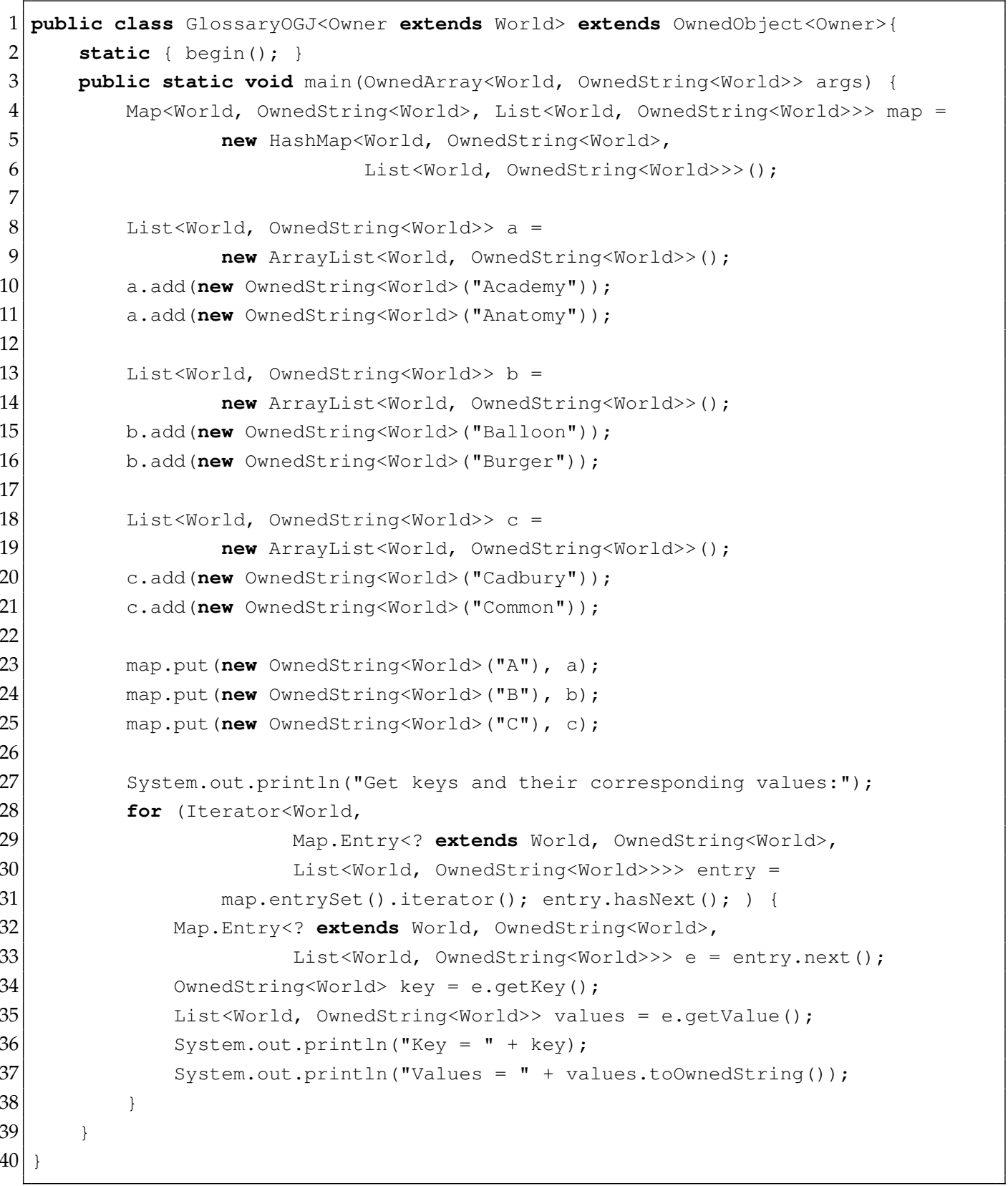

Figure 5.19: $O \mathrm{GJ}_{+}$'s version of Fig. 5.18 . 
118CHAPTER 5. GENERIC OWNERSHIP COMPLIANT COLLECTIONS 


\section{Chapter 6}

\section{Evaluation}

To evaluate the refactored collections in terms of performance, we conducted some micro-benchmark tests comparing the performance time of the original and refactored collections.

As explained in the previous chapter, all class headers of the refactored collections are no longer compatible with those of the JDK 1.6 collections; all classes must involve owner parameters; all type variables are bounded by IOwnedObject; Object is no longer permitted as a type in $\mathrm{OGJ}_{+}$; a significant number of method signatures have been changed; Java's class types, such as String and Integer, have been wrapped in owned classes; Java arrays are no longer permitted. As a result, any benchmark suite that is intended as a tool for Java benchmarking needs to be refactored, in order to benchmark our refactored collections.

We found that refactoring a macro-benchmark, such as DaCapo or SPECjvm2008, would be another big project; and in the end, we do not expect that collections form a considerable share of their execution. Accordingly, we decided to modify four of Doug Lea's JRS-166 [33] micro-benchmarks that were specially implemented to target the Collections Framework. These micro-benchmarks are IteratorLoops, CollectionLoops, MapLoops and MapMicroBenchmark. The latter is in fact implemented to represent realistic uses of the Map classes. Since 
Doug Lea is a co-developer of the Collections Framework, this set of micro-benchmarks is considered the best for experimenting the collections' performance.

All benchmarks were modified to be compatible with the class headers and method signatures of the $\mathrm{OGJ}_{+}$'s collection classes. Class types (e.g., Integer) were replaced with their corresponding types in $O G J_{+}$. The modified micro-benchmarks were used along with the original ones to compare the performance of our refactored collections against the JDK 1.6 collections. Some changes have been applied to the behaviour of both the $\mathrm{OGJ}_{+}$compliant benchmarks and the original benchmarks. These changes are explained in detail, where applicable, in the following sections.

All benchmarks were run using the Java Platform JDK 1.6.0_30 (Java HotSpot 64-Bit Server VM 20.5-b03) on the NetBeans IDE 7.1 (Build 201112071828). The computer used has a 64-bit Intel chip (Core i3 at 2.13 $\mathrm{GHz}$ ) and 4GB of RAM, running a 64-bit operating system (Microsoft Windows version 8 ). In the rest of this chapter, we present some experimental results.

\subsection{IteratorLoops}

IteratorLoops measures the performance time for each step of the iteration process. IteratorLoops can test Collection classes as well as Map classes. The estimates are provided in nanoseconds per each iteration step; that is, the time needed to execute a single next ( ) operation call. IteratorLoops runs 268,435,456 iteration steps over an array of 16 Collection objects of size 16384 (the square root of the iteration steps). IteratorLoops intermittently adds about $1 / 8$ of the preloaded elements at run-time.

Fig. 6.1 shows the estimates for all of our refactored collections and maps against the original ones. The plots in Fig. 6.1 are the average of four trials per run per collection class. This is the default number of trials IteratorLoops produce. We executed IteratorLoops several times 


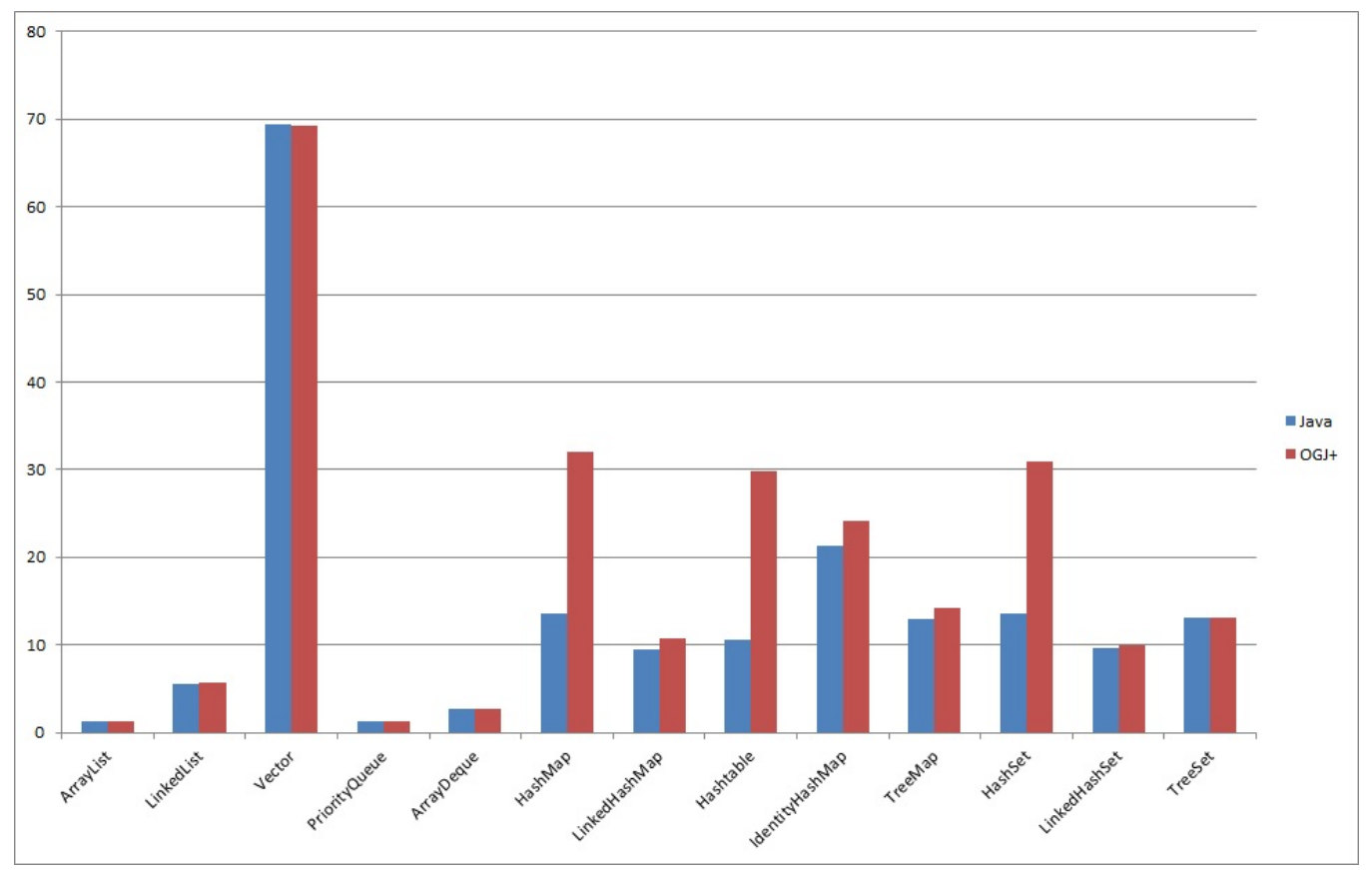

Figure 6.1: IteratorLoops - Nanoseconds per Iteration Step

for each collection class and found that the produced numbers are relatively stable.

Except for Hashset, all of the other refactored collection classes show no variance of performance degradation. As for the Map classes, LinkedHashMap, Ident ityHashMap and TreeMap show no significant variance (1-3 nanoseconds) of performance degradation. The performance of Hashtable and HashMap (and hence HashSet) is around 2.5 times slower. This is mainly because of the way the next () method works. In HashMap, the backing array of entries is not a contiguous store; there could be null values in between. The next () method performs a sequential walk over the backing array until a non-nul 1 value is found. The walking loop used by the next () method of the original HashMap is as follows:

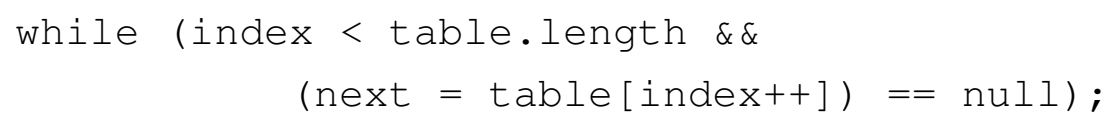


Java keeps type information at runtime for array types. Java arrays are eliminated in $\mathrm{OGJ}_{+}$that uses the generic type OwnedArray. With generic types, type information is not kept at runtime; the compiler inserts checkcast instructions (The bytecode instructions for performing casting at runtime). That is, every walking step performed by the next () method of the refactored HashMap requires a checkcast to check if the object extracted from OwnedArray could be cast to the type of next. This should increase the time needed to execute the next () operation. The same applies to Hashtable.

\subsection{CollectionLoops}

CollectionLoops can test only Collection classes. CollectionLoops is originally implemented to exercise multi-threaded collections; but for an unknown reason, some Col lection classes (original and refactored) cannot complete the test. We modified collectionLoops to exercise single-threaded collections. A thread does a random walk over an array of 10,000 elements. On each iteration, CollectionLoops checks if the collection contains the given element. If the element is absent, CollectionLoops adds it. If the element is present, CollectionLoops removes it. CollectionLoops performs 100,000 operations per trial. A trial measures the performance time for each operation in nanoseconds.

By default, Collecti onLoops runs four trials for single-threaded collections. With set classes (refactored and original), we noticed that the numbers of the first two trials are significantly higher than those of the last two trials; and the numbers of the first two trials significantly vary from run to run. We decided to loop the four trials 25 times, and the output was 52 trials. We modified collectionLoops to calculate the average of the 52 results. With the HashSet and LinkedHashSet, we found after several runs that the averages vary from 65 to 85 nanseconds per operation, and more than $90 \%$ of the 52 results are under or between 
these two averages. Proportionally, the same happens with Treeset. Although the other Collection classes produce stable results, we kept CollectionLoops with these modifications, and tested all of the collection classes. For the Set classes, the average of the best five repetitions was calculated to represent the performance time per operation.

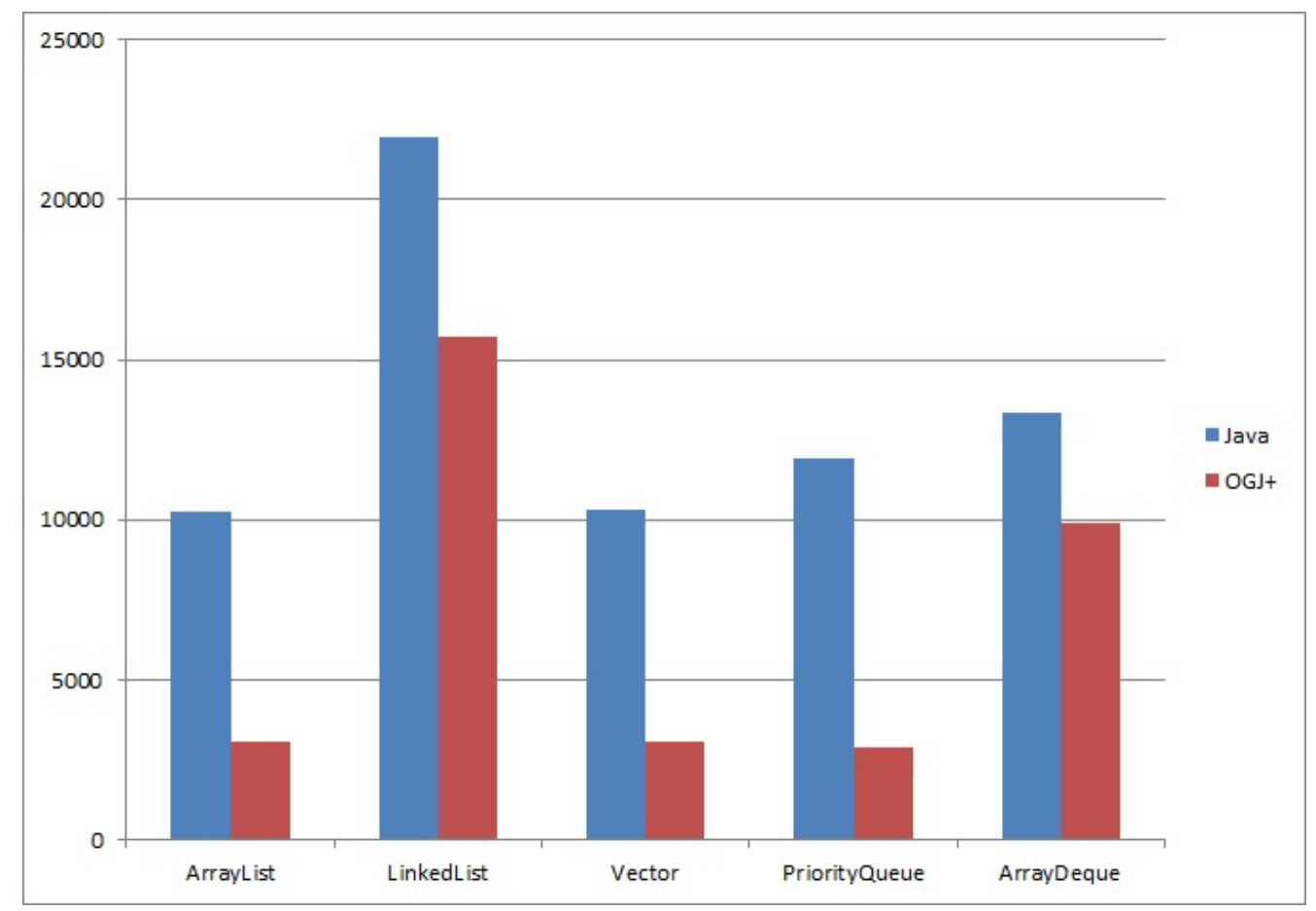

Figure 6.2: CollectionLoops for Lists and Queues - Nanoseconds per Operation

Fig. 6.2 shows the results for the lists and queues. There is a significant improvement in the performance of the refactored classes. This is because there is a significant decline in the number of implicit type casts. To explain this, we first recall that the original ArrayList, Vector and Priorityqueue are backed by arrays of type object, while the corresponding refactored versions are backed by OwnedArray instances that store objects of type $\mathrm{E}$, where $\mathrm{E}$ is the type variable of the collection class. ArrayDeque is originally backed by an array of type $\mathrm{E}$, and the nodes of 
LinkedList originally hold elements of type E. Note that the improvement in the performance of the refactored versions of the latter two implementations is not as great as that of the other three implementations. In the five classes, all of the methods that originally take ob ject as an argument were refactored to take $\mathrm{E}$ as an argument (e.g., contains (), indexOf (), remove()). CollectionLoops uses contains() very heavily. The implementation of contains (), in any of the five collections, checks if the specified object (method argument) is contained in the current collection by iterating over all of the stored objects to see whether one of them is equivalent to the specified object using the equals () method. Except for ArrayDeque, contains () delegates lookups to indexOf (). The indexOf () method (or contains () in case of ArrayDeque) walks the backing array (or the linked list) using a for loop. Each step invokes equals (). If both the receiver and the argument of equals ( ) are of type $\mathrm{E}$, then the number of the required implicit casts will be less than the number of implicit casts required to complete the operation with a receiver or argument (or both) of type object.

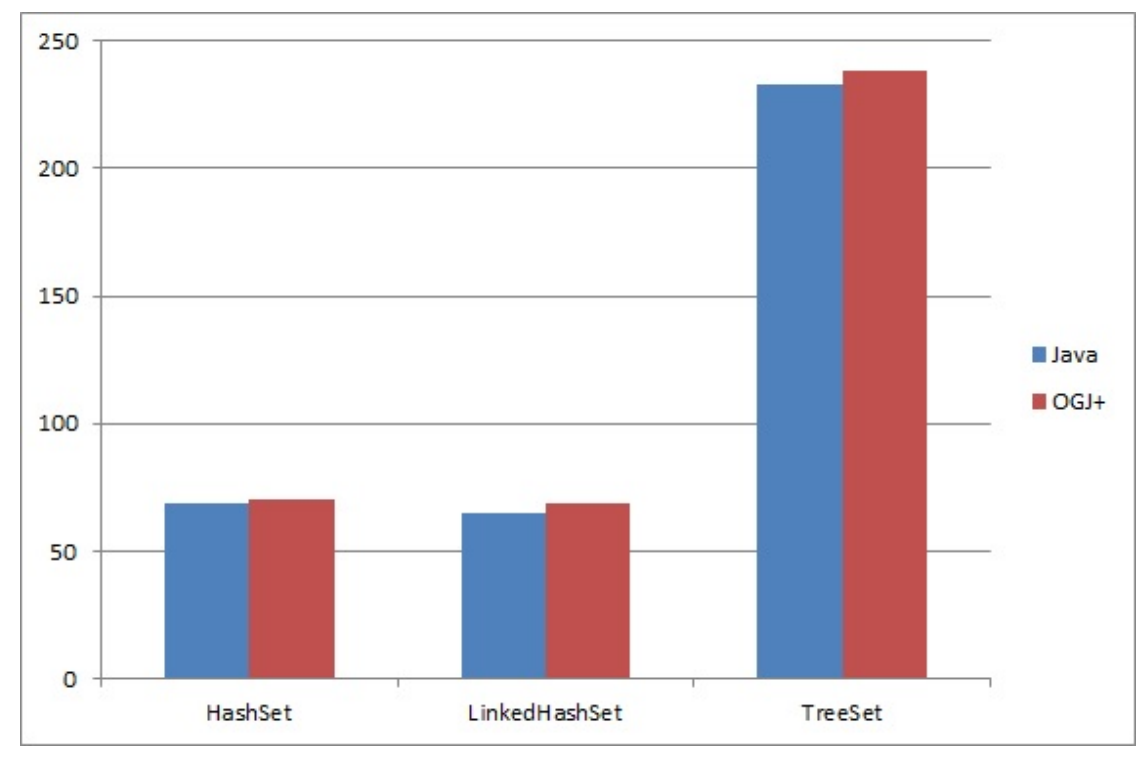

Figure 6.3: CollectionLoops for Sets - Nanoseconds per Operation 
Fig. 6.3 shows the results for the set classes. The plots show no significant variance (1-5 nanoseconds) of performance degradation. The contains() method of a set implementation delegates lookups to containsKey () of the backing Map implementation. With respect to the hash-based Map implementations, containskey () uses the hash of the key to go directly to the position where this key should reside and then uses equals () to check if it is there. That is, equals () is not involved in a lookup iteration process, of which each step requires a call to equals (); and thus, the performance of the refactored sets is just as the original sets. The containskey () of TreeMap does not use equals () to determine equality; it uses Comparable. compareTo (). In contrast to a binary method such as equals (), the receiver type and the argument type of compareTo () do not need to coincide. Moreover, the argument type of compareTo () is $\mathrm{K}$ (the variable type for the keys) in both the refactored and the original TreeMap classes. That is, in both versions there is a single implicit cast required for each invocation during the lookup; and thus, the performance of the refactored Treeset is similar to that of the original Treeset. 


\subsection{MapLoops}

MapLoops can test only Map classes. Similar to CollectionLoops, MapLoops is originally implemented to exercise multi-threaded maps, but we modified it to exercise single-threaded maps and to exercise the same number of operations and trials as CollectionLoops. Similar to what we did with Set classes, MapLoops were repeatedly run, then the mean of the best five repetitions were calculated to represent the performance time per operation. The plots in Fig. 6.4 show that the performance of the refactored maps is not significantly different from that of the original maps.

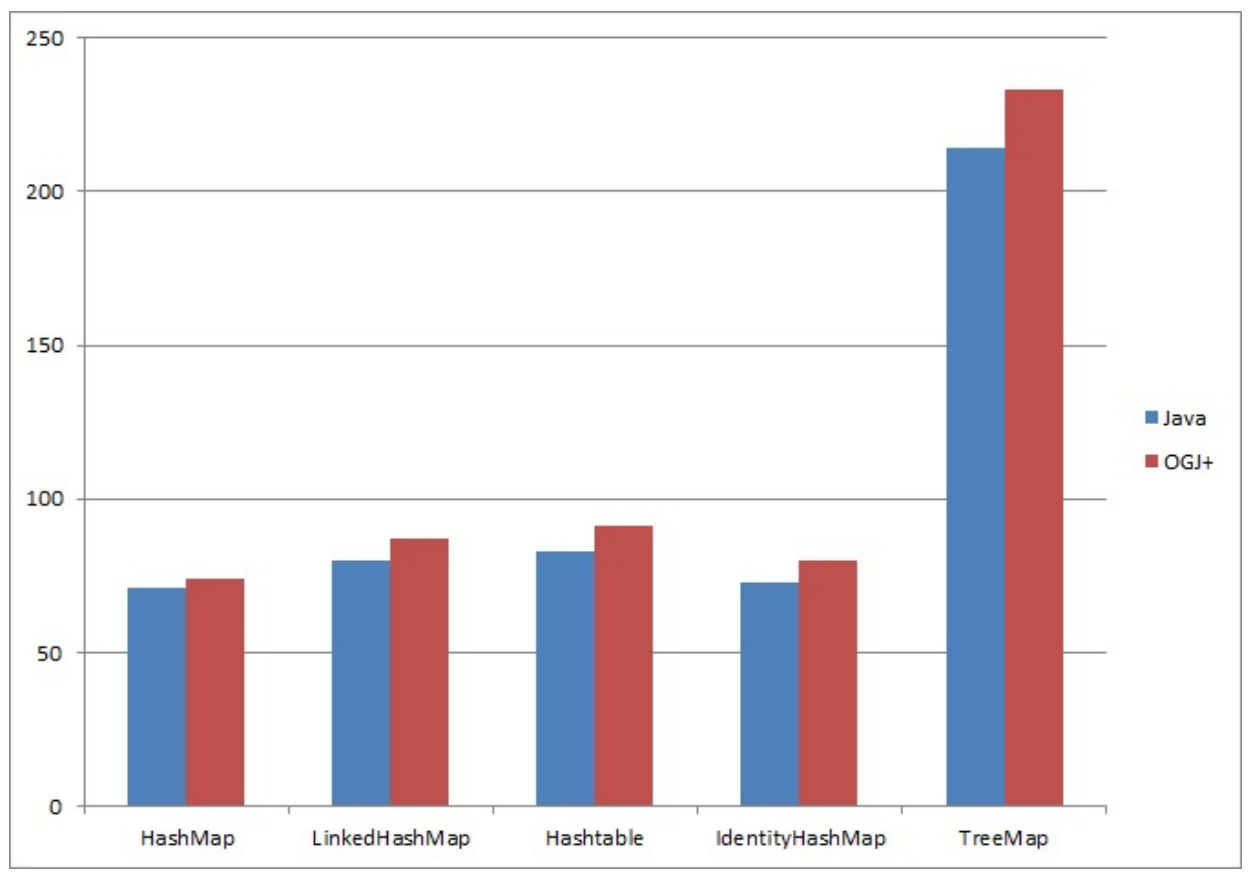

Figure 6.4: MapLoops - Nanoseconds per Operation 


\subsection{MapMicroBenchmark}

MapMicroBenchmark is another maps specific benchmark, but it cannot run maps that do not permit insertion of non-comparable objects (objects that do not implement the Comparable interface). Therefore, we couldn't test TreeMap with MapMicroBenchmark. According to the very brief documentation provided, this is "a micro-benchmark with key types and operation mixes roughly corresponding to some real programs". "The main results are a table of approximate nanoseconds per elementoperation (averaged across get, put etc) for each type, across a range of map sizes"; see Fig. 6.6. We did not make any modification to the default values of this class. The plots in Fig. 6.5 represent the averages calculated for the largest map size (see the boxed number in the lower-right corner of Fig. 6.6. As shown, the performance of the refactored maps is almost the same as that of the original maps.

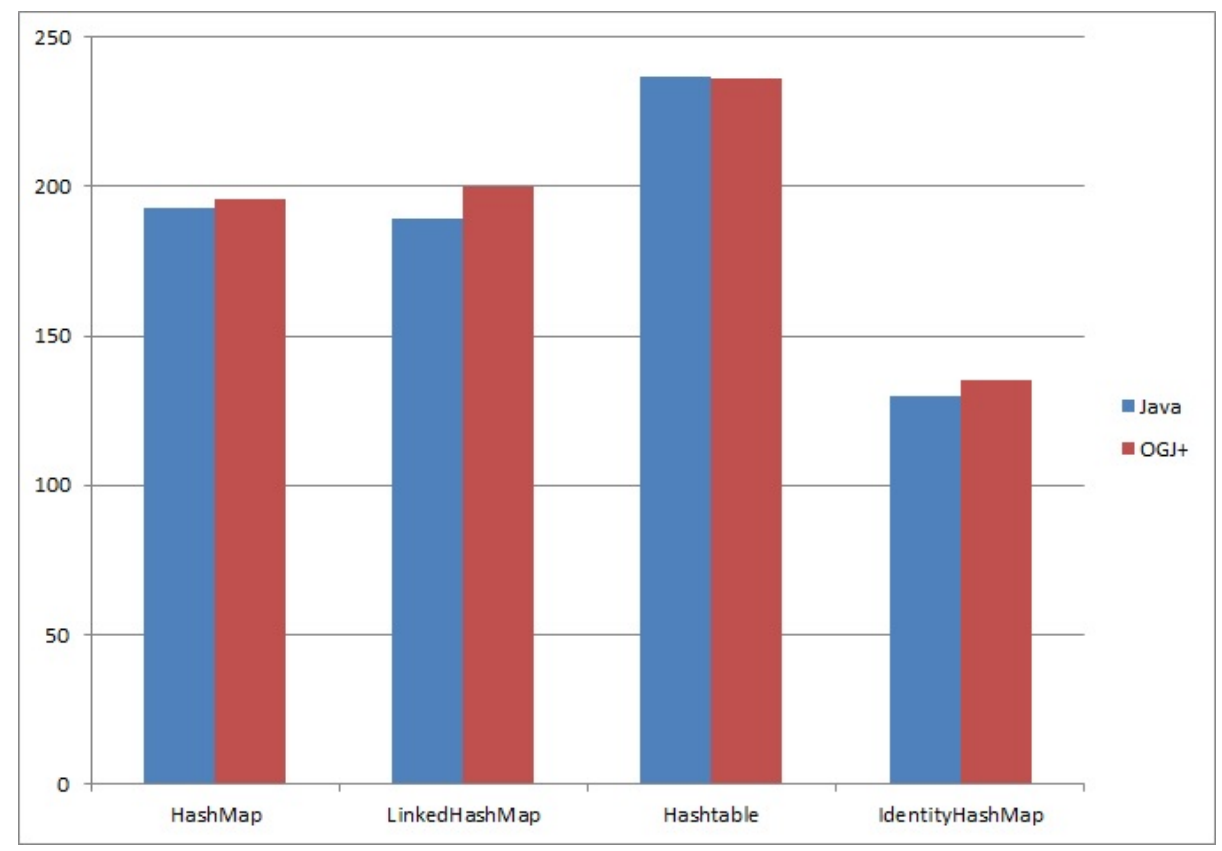

Figure 6.5: MapMicroBenchmark - Nanoseconds per Operation over a map size of 589,824 


\begin{tabular}{|c|c|c|c|c|c|c|c|c|c|}
\hline $\begin{array}{l}\text { Class java. } \\
\text { No word fil } \\
\text { warm up... } \\
\text { warm up... } \\
\text { running.... }\end{array}$ & $\begin{array}{l}\text { 1.Ha } \\
\text { Usin }\end{array}$ & $\begin{array}{l}\text { ap } \\
\text { tri }\end{array}$ & $\begin{array}{l}\text { omi } \\
\text { alu } \\
\ldots\end{array}$ & $\begin{array}{l}\text { sear } \\
\text { (i) }\end{array}$ & רes & $\ldots$ & ....... & $\ldots \ldots$ & $\begin{array}{c}\ldots \ldots \ldots \\
\cdots \ldots \ldots \ldots \\
\ldots \ldots \ldots\end{array}$ \\
\hline Type/Size: & 9 & 36 & 144 & 576 & 2304 & 9216 & 36864 & 147456 & 589824 \\
\hline object & 24 & 24 & 24 & 26 & 29 & 40 & 52 & 140 & 175 \\
\hline String & 22 & 24 & 24 & 27 & 28 & 44 & 97 & 167 & 191 \\
\hline Integer & 32 & 31 & 31 & 30 & 32 & 40 & 52 & 129 & 162 \\
\hline Long & 23 & 21 & 21 & 21 & 23 & 31 & 42 & 120 & 155 \\
\hline Float & 29 & 25 & 29 & 30 & 31 & 36 & 56 & 133 & 187 \\
\hline Double & 24 & 27 & 26 & 25 & 33 & 39 & 50 & 148 & 186 \\
\hline BigInteger & 29 & 30 & 29 & 30 & 35 & 47 & 110 & 181 & 213 \\
\hline BigDecimal & 25 & 31 & 31 & 31 & 35 & 49 & 79 & 173 & 199 \\
\hline RandomInt & 37 & 36 & 37 & 37 & 41 & 53 & 71 & 177 & 219 \\
\hline Mixed & 35 & 38 & 43 & 48 & 58 & 72 & 152 & 212 & 249 \\
\hline average & 28 & 28 & 29 & 30 & 34 & 45 & 76 & 158 & 193 \\
\hline
\end{tabular}

Figure 6.6: MapMicroBenchmark's results for JDK 1.6 HashMap

\subsection{Discussion}

Merging ownership information into generic declarations does not impose significant runtime overheads, if there are any. Since all information about generics is erased by the compiler after all checks are done, Java generics might seem to be implemented with high overhead, requiring implicit upcasts to Ob ject in the process of inserting elements into a collection, and implicit downcasts to the type of the elements in the process of taking the elements out of the given collection. This is not precise, however. The fact is that the implementations of some Collection classes involve arrays of type Ob ject rather than E. In the refactored collections, all of the backing arrays became of type $E$, which is the type variable of their respective collection classes. We did not voluntarily choose to make the backing arrays hold elements of type E. As explained in subsection 5.1.1. we had no choice but to make the methods, which take Object as an argument, take $\mathrm{E}$ as an argument due to the owner nesting rule imposed by $O G J_{+}$. Similarly, the way to make the owner of the backing array elements the same as the owner of the collection elements is by making the type of the 
backing array the same as the type of the collection elements, given that Java's "no generic array creation" property is no longer a concern to $O G J_{+}$.

There is no doubt that Java generics impose runtime overheads which we notice clearly as we test the refactored HashMap class with IteratorLoops. Walking an array that preserves its type information at runtime is more efficient than walking a generic aggregate such as OwnedArray. The use of array types, which do not hold elements of concrete parameterized types, to back collection classes, which accept elements of concrete parameterized types, has in fact eased the cost of Java generics.

Merging ownership information into generic declarations necessitated the prevention of raw types and unbounded wildcard parameterized types; both kinds of types are permitted as types of Java arrays. Preserving the owners of both the backing aggregate and its component type is an uncompromised priority in $\mathrm{OGJ}_{+}$. By using OwnedArray as a workaround for the nonexistent generic array type, various settings have changed in both the implementation of the collection classes and their generated byte code. In addition to the added runtime checkcast instructions and implicit casts, operations such as OwnedArray - get () and OwnedArray . set () had to replace the array index operator. Such operations, with others, should impose runtime overheads. Nevertheless, the experimental results we presented suggest that the cost of combing ownership types with parametric polymorphism is relatively low, and can even be avoided by takeing alternative implementation decisions.

$\mathrm{OGJ}_{+}$has the ability to enforce certain implementation decisions in favour of efficiency. For example, with the original collections one can query if an orange is contained in a basket of apples, and the answer is false because such a query is allowed; but with the refactored collections, such a query is not allowed. Our experiments turned out that allowing this kind of queries with Java generics is expensive. For $O G J_{+}$to confirm that owner nesting is preserved, the possibility of allowing this kind of queries is very limited; and if the owner of the component type is sub- 
ject to context covariance, as is the case with the refactored collections, the possibility does not exist. Indeed, making an owner subject to context covariance is our decision, but maintaining readonly wildcard owner parameterised references from inner classes to their outer classes necessitated this decision. Otherwise, we should have made the component type owned by World, which is not a reasonable decision.

The results of MapMicroBenchmark confirm that real programs would not be affected by the overhead IteratorLoops produced for HashMap. Based on the entire experimental results, we believe that the $O G J_{+}$prototype has confirmed expectations, given that Java generics current implementation does not touch the JVM or the class file structure. Emplacing ownership information into generic declarations, with the required workarounds, might keep the question open: what does matter more, security or efficiency? We believe, however, that the cost of security is not high, based on these results. 


\section{Chapter 7}

\section{Conclusions}

Supporting efficient, true and handy encapsulation of software components and their associated data types is a lively concern in object-oriented research. In this thesis we have presented $\mathrm{OGJ}_{+}$, an actual usable language implementation with deep ownership types extended with support for readonly references. $\mathrm{OGJ}_{+}$extends Java with a form of statically enforceable instance-level hierarchical encapsulation, rather than the classlevel private field encapsulation. We claim to have taken advantage of Java Generics to the extreme. We have utilised the safe form of covariance provided by the wildcard feature of Java generics to provide flexible referencing without compromising the encapsulation or the nested structure of the heap. Some features, such as inner classes, equals, statics and exception handling, would have been impaired under the strict model of deep ownership, if wildcards were not utilised to favour ownership.

$\mathrm{OGJ}_{+}$proved to have the potentials of supporting safe programming with aliasing. For the practical evaluation, we refactored an essential portion of the Collections Framework, showing that programming with OGJ will not hamper the construction of realistic software. While maintaining the expressiveness of aliasing properties of the fields, OGJ + was able to precisely detect all breaches of encapsulation. We showed that the refactoring was made without violating the traditional Java programming 
style; programmers are still able to take advantage of the full potentials of inner classes. Therefore, we believe we managed to find a viable solution for the iterator pattern. In comparison with the ad hoc approach proposed by Boyapati et al. [9], OGJ + does not permit an inner class and its outer class to share a common representation; ownership information is strictly class-specific. The internal representation of an outer class can only be referred to, form an inner class, through wildcard-owned references, which are readonly.

During the refactoring, $\mathrm{OGJ}$ + showed its ability to enforce constraints on data structures; during the experiments, these constraints proved to mitigate or reduce potential negative effects of implicit casting. Nevertheless, the nature of generic ownership as a language level mechanism associated with Java generics makes all of our solutions coupled with the effects and side effects of Java generics.

The undesired effects of aliasing (see example in Fig. 2.2) are known as a problem [31] that programmers should be aware of. Researchers suggest that thinking about ownership structures can lead to better code structures [58]. We claim that programming with $O G J_{+}$allows only positive thoughts of aliasing, and requires knowledge about the meaning of ownership types.

\subsection{Limitations}

The most notable limitation of $O G J_{+}$is that the provided stronger encapsulation guarantees comes at the cost of programming overhead. We discussed in the thesis a number of limitations that might impact the way software is written in $O G J_{+}$. Section 3.5 describes how casting is treated in $\mathrm{OGJ}_{+}$and explains the idea of using wildcards to overcome the limitation of not keeping track of the owners at run-time. Since casting cannot be dealt with in isolation from the equals idiom, section 4.5 takes the discussion further afield than in section 3.5. Section 4.2 describes a limita- 
tion with the event-listeners design pattern; the pattern can still be implemented in $\mathrm{OGJ}_{+}$, however. Subsection 5.4.2 highlights another limitation with the template-method design pattern. Note that the aim of this thesis project is not to evaluate $O G J_{+}$with design patterns. Finally, section 4.4 involves a discussion on the restrictive nature of $\mathrm{OGJ}_{+}$'s deep cloning.

\subsection{Future Directions}

We will continue to improve $\mathrm{OGJ}_{+}$and evaluate it in large programs. We think of evaluating the language with the concurrent implementations of the Collections Framework. We are also interested in evaluating the language with design patterns and see if more extensions would be required. Initially, we think that a feature for ownership transfer [48] would be a great addition to the language. Another interesting future work might be allowing an object to have multiple owners [14].

We also think that relying on genericity should not be everything for $\mathrm{OGJ}_{+}$. Finding a more reasonable solution for arrays would be a great restoration. Supporting immutability could also be an interesting future work, in this regard. 


\section{Bibliography}

[1] Collections framework overview. In Java SE Documentation, Oracle Technology Network. Available Online: http://download.oracle. com/ javase/6/docs/technotes/guides/collections/overview.html. Viewed 16 Apr 2011.

[2] Java Collections API Design FAQ. In Java SE Documentation, Oracle Technology Network. Available Online: http://download.oracle. $\mathrm{com} /$ javase/6/docs/technotes/guides/collections/designfaq.html. Viewed 26 May 2011.

[3] Aldrich, J., And Chambers, C. Ownership domains: Separating aliasing policy from mechanism. In ECOOP '04 - Object-Oriented Programming European Conference (Oslo, Norway, 2004), M. Odersky, Ed., vol. 3086 of Lecture Notes in Computer Science, Springer-Verlag, pp. $1-25$.

[4] AlmeidA, P. S. Balloon types: Controlling sharing of state in data types. In ECOOP (1997), pp. 32-59.

[5] BLOCH, J. Collections. In The Java Tutorials, Oracle Technology Network. Available Online: http://download.oracle.com/ javase/tutorial/collections/. Viewed 16 Apr 2011.

[6] Booch, G., MaksimchuK, R., Engle, M., Young, B., Conallen, J., AND Houston, K. Object-oriented analysis and design with applications, third edition, third ed. Addison-Wesley Professional, 2007. 
[7] BOYAPATI, C. SafeJava : a unified type system for safe programming. $\mathrm{PhD}$ thesis, Massachusetts Institute of Technology, 2004.

[8] Boyapati, C., LeE, R., AND Rinard, M. Safe runtime downcasts with ownership types. In International Workshop on Aliasing, Confinement and Ownership in Object-oriented Programming (July 2003), D. Clarke, Ed., UU-CS-2003-030, Utrecht University.

[9] BoyApati, C., LiskOv, B., AND SHRIRA, L. Ownership types for object encapsulation. In Proceedings of the 30th ACM SIGPLAN-SIGACT symposium on Principles of programming languages (New York, NY, USA, 2003), POPL '03, ACM, pp. 213-223.

[10] BOYLAND, J. Alias burying: Unique variables without destructive reads. Software-Practice and Experience 31, 6 (May 2001), 533-553.

[11] Boyland, J., Noble, J., AND Retert, W. Capabilities for sharing: A generalisation of uniqueness and read-only. In ECOOP '01: Proceedings of the 15th European Conference on Object-Oriented Programming (London, UK, 2001), Springer-Verlag, pp. 2-27.

[12] Cameron, N., AND Drossopoulou, S. Existential quantification for variant ownership. In Proceedings of the 18th European Symposium on Programming Languages and Systems: Held as Part of the Joint European Conferences on Theory and Practice of Software, ETAPS 2009 (Berlin, Heidelberg, 2009), ESOP '09, Springer-Verlag, pp. 128-142.

[13] CAmeron, N., AND Noble, J. Ogj gone wild. In International Workshop on Aliasing, Confinement and Ownership in Object-Oriented Programming (New York, NY, USA, 2009), IWACO '09, ACM, pp. 7:1$7: 10$.

[14] Cameron, N. R., Drossopoulou, S., Noble, J., And Smith, M. J. Multiple ownership. In Proceedings of the 22nd annual ACM SIGPLAN 
conference on Object-oriented programming systems and applications (New York, NY, USA, 2007), OOPSLA '07, ACM, pp. 441-460.

[15] CAMERON, N. R., AND Noble, J. Encoding ownership types in java. In TOOLS (48) (2010), J. Vitek, Ed., vol. 6141 of Lecture Notes in Computer Science, Springer, pp. 271-290.

[16] Clarke, D. Object Ownership \& Containment. PhD thesis, University of New South Wales, 2001.

[17] Clarke, D., AND Drossopoulou, S. Ownership, encapsulation and the disjointness of type and effect. In Proceedings of the 17th ACM SIGPLAN conference on Object-Oriented Programming, Systems, Languages, and Applications (New York, NY, USA, 2002), OOPSLA '02, ACM, pp. 292-310.

[18] Clarke, D., Shelswell, R., Potter, J., and Noble, J. Object ownership to order. Tech. rep., Microsoft Research Institute, 1998.

[19] Clarke, D., AND Wrigstad, T. External uniqueness is unique enough. In In European Conference for Object-Oriented Programming (ECOOP (2003), Springer-Verlag, pp. 176-200.

[20] Clarke, D. G., Noble, J., and Potter, J. Overcoming representation exposure. In ECOOP Workshops (1999), A. M. D. Moreira and S. Demeyer, Eds., vol. 1743 of Lecture Notes in Computer Science, Springer, pp. 149-151.

[21] Clarke, D. G., Potter, J. M., And Noble, J. Ownership Types for Flexible Alias Protection. ACM SIGPLAN Notices 33, 10 (Oct. 1998), 48-64.

[22] Detlefs, D. L., Leino, K. R. M., AND Nelson, G. Wrestling with rep exposure. SRC Research Report 156, Compaq Systems Research Center, 130 Lytton Ave., Palo Alto, July 1998. 
[23] Dietl, W., Drossopoulou, S., AND Müller, P. Generic universe types. In ECOOP (2007), E. Ernst, Ed., vol. 4609 of Lecture Notes in Computer Science, Springer, pp. 28-53.

[24] Dietl, W., AND MÜller, P. Exceptions in ownership type systems. In ECOOP Workshop FTfJP'2004 Formal Techniques for Java-like Programs (June 2004), E. Poll, Ed., pp. 49-54.

[25] DieTL, W., AND MÜLLER, P. Universes: Lightweight ownership for JML. Journal of Object Technology 4, 8 (2005), 5-32.

[26] Eliëns, A. Principles of Object-Oriented Software Development, second ed. Addison-Wesley, 2000.

[27] FAhndrich, M., AND Deline, R. Adoption and focus: practical linear types for imperative programming. SIGPLAN Not. 37, 5 (2002), 13-24.

[28] Gosling, J., Joy, B., Steele, G., AND Bracha, G. Java(TM) Language Specification, The (3rd Edition) (Java (Addison-Wesley)). AddisonWesley Professional, 2005.

[29] Grogono, P., And Sakkinen, M. Copying and comparing: Problems and solutions. In Proceedings of the 14th European Conference on Object-Oriented Programming (2000), ECOOP '00, Springer-Verlag, pp. 226-250.

[30] HoGG, J. Islands: aliasing protection in object-oriented languages. ACM SIGPLAN Notices 26, 11 (Nov. 1991), 271-285.

[31] Hogg, J., Lea, D., Wills, A., DeChampeaux, D., And Holt, R. The Geneva convention on the treatment of object aliasing. SIGPLAN OOPS Mess. 3, 2 (1992), 11-16. 
[32] Huang, W., AND Milanova, A. Towards effective inference and checking of ownership types. In Proceedings of the International Workshop on Aliasing, Confinement and Ownership at ECOOP (IWACO 2011) (July 2011).

[33] LEA, D. Jsr-166 loops microbenchmarks. http://gee.cs.oswego.edu/ cgi-bin/viewcrs.cgi/jsr166/src/test/loops/.

[34] Leavens, G. T., BAKer, A. L., AND Ruby, C. JML: A notation for detailed design. In Behavioral Specifications of Businesses and Systems, H. Kilov, B. Rumpe, and I. Simmonds, Eds. Kluwer Academic Publishers, Boston, 1999, pp. 175-188.

[35] Leavens, G. T., BaKer, A. L., And Ruby, C. Preliminary design of JML: A behavioral interface specification language for Java. Tech. Rep. 98-06y, Iowa State University, Department of Computer Science, 2003. Revised June 2004.

[36] Leavens, G. T., Poll, E., Clifton, C., Cheon, Y., Ruby, C., CoK, D. R., Müllek, P., Kiniry, J., Chalin, P., Zimmerman, D. M., AND DieTL, W. JML reference manual. Available from http:// Www. jmlspecs.org, May 2008.

[37] Leino, K. R. M., AND STATA, R. Virginity: a contribution to the specification of object-oriented software. Inf. Process. Lett. 70, 2 (Apr. 1999), 99-105.

[38] Li, P., CAmeron, N., And Noble, J. Cloning in ownership. In Proceedings of the ACM international conference companion on Object oriented programming systems languages and applications companion (New York, NY, USA, 2011), SPLASH '11, ACM, pp. 63-66.

[39] Liskov, B., AND GutTAG, J. Abstraction and specification in program development. MIT Press, Cambridge, MA, USA, 1986. 
[40] Liskov, B., SNyder, A., AtKinson, R., And Schaffert, C. Abstraction mechanisms in CLU. Commun. ACM 20, 8 (August 1977), $564-576$.

[41] LisKOV, B., AND ZILles, S. Programming with abstract data types. In ACM SIGPLAN Conference on Very High Level Languages, SIGPLAN Notices (Apr. 1974), vol. 9, pp. 50-59.

[42] Meyer, B. Object-oriented software construction (2nd ed.). Prentice-Hall, Inc., Upper Saddle River, NJ, USA, 1997.

[43] Minsky, N. H. Towards alias-free pointers. In ECCOP '96: Proceedings of the 10th European Conference on Object-Oriented Programming (London, UK, 1996), Springer-Verlag, pp. 189-209.

[44] Müller, P. Modular Specification and Verification of Object-Oriented Programs, vol. 2262 of Lecture Notes in Computer Science. SpringerVerlag, 2002.

[45] Müller, P., ANd Poetzsch-Heffter, A. Universes: A type system for controlling representation exposure. In Programming Languages and Fundamentals of Programming (1999), A. Poetzsch-Heffter and J. Meyer, Eds., Fernuniversität Hagen.

[46] Müller, P., And Poetzsch-Heffter, A. A type system for controlling representation exposure in Java. In Formal Techniques for Java Programs (2000), S. Drossopoulou, S. Eisenbach, B. Jacobs, G. T. Leavens, P. Müller, and A. Poetzsch-Heffter, Eds., Technical Report 269, Fernuniversität Hagen.

[47] Müller, P., ANd Poetzsch-Heffter, A. Universes: A type system for alias and dependency control. Tech. Rep. 279, Fernuniversität Hagen, 2001. 
[48] MÜller, P., AND RUdich, A. Ownership transfer in universe types. In OOPSLA '07: Proceedings of the 22nd annual ACM SIGPLAN conference on Object-oriented programming systems and applications (New York, NY, USA, 2007), ACM, pp. 461-478.

[49] Naftalin, M., AND WAdleR, P. Java Generics and Collections. O’Reilly Media, Inc., 2006.

[50] NÄGELI, S. Ownership in design patterns. Master's thesis, Software Component Technology Group, Department of Computer Science, ETH Zurich, 2006.

[51] Noble, J., Clarke, D., AND Potter, J. Object ownership for dynamic alias protection. In Proceedings TOOLS '99 (Nov. 1999).

[52] Noble, J., Vitek, J., And Potter, J. Flexible Alias Protection. In ECOOP'98-Object-Oriented Programming (1998), E. Jul, Ed., vol. 1445 of Lecture Notes in Computer Science, Springer, pp. 158-185.

[53] Pagh, A., PAGH, R., And Ruzic, M. Linear probing with constant independence. In Proceedings of the thirty-ninth annual ACM symposium on Theory of computing (New York, NY, USA, 2007), STOC '07, ACM, pp. 318-327.

[54] Parnas, D. L. On the criteria to be used in decomposing systems into modules. Commun. ACM 15, 12 (Dec. 1972), 1053-1058.

[55] PARnas, D. L. A technique for software module specification with examples. Commun. ACM 15, 5 (May 1972), 330-336.

[56] PIERCE, B. C. Types and programming languages. MIT Press, Cambridge, MA, USA, 2002.

[57] Potanin, A. Generic Ownership: A Practical Approach to Ownership and Confinement in OO Programming Languages. $\mathrm{PhD}$ thesis, Victoria University of Wellington, 2007. 
[58] Potanin, A., Damitio, M., And Noble, J. Are your incoming aliases really necessary? counting the cost of object ownership. In Proceedings of the 2013 International Conference on Software Engineering (Piscataway, NJ, USA, 2013), ICSE '13, IEEE Press, pp. 742-751.

[59] Potanin, A., Noble, J., Clarke, D., And Biddle, R. Defaulting Generic Java to Ownership. In In Proceedings of the Workshop on Formal Techniques for Java-like Programs in European Conference on ObjectOriented Programming (FTfJP (2004), Springer-Verlag.

[60] Potanin, A., Noble, J., Clarke, D., And Biddle, R. Generic ownership. In In 7th Workshop on Formal Techniques for Java-like Programs - FTfJP2005 (2004).

[61] Potanin, A., Noble, J., Clarke, D., And Biddle, R. Generic Ownership for Generic Java. In Proceedings of the 21st annual ACM SIGPLAN conference on Object-Oriented Programming Systems, Languages, and Applications (New York, NY, USA, 2006), OOPSLA '06, ACM, pp. 311-324.

[62] Potter, J., Noble, J., And Clarke, D. The ins and outs of objects. In Proceedings of the Australian Software Engineering Conference (Washington, DC, USA, 1998), ASWEC '98, IEEE Computer Society, pp. 80-.

[63] Summers, A. J., Drossopoulou, S., AND Müller, P. Universetype-based verification techniques for mutable static fields and methods. Journal of Object Technology 8, 4 (2009), 85-125.

[64] ViteK, J., AND BoKOwsKi, B. Confined types in Java. Software Practice and Experience 31, 6 (2001), 507-532.

[65] WADLER, P. Linear types can change the world! In Working Conference on Programming Concepts and Methods (Sea of Galilee, Israel, April 1990), IFIP TC 2. 
[66] Wegner, P. Dimensions of object-based language design. In Conference proceedings on Object-oriented programming systems, languages and applications (New York, NY, USA, 1987), OOPSLA '87, ACM, pp. 168182.

[67] Wrigstad, T. Ownership-Based Alias Management. PhD thesis, Royal Institute of Technology, Sweden, May 2006.

[68] Wrigstad, T., AND Clarke, D. Existential owners for ownership types. Journal of Object Technology 6, 4 (2007). 